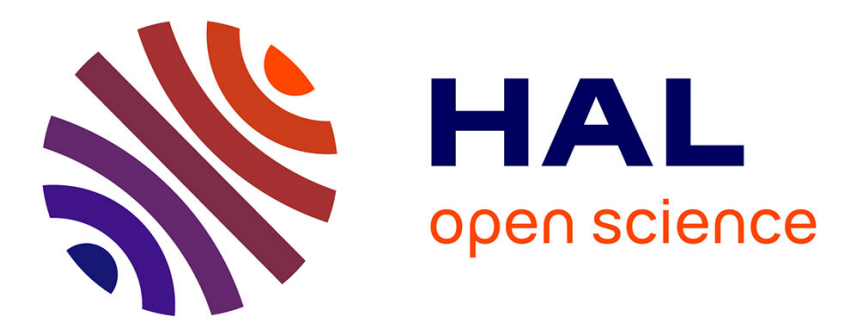

\title{
Pyroelectric-controlled bending of a self-trapped optical beam in a photorefractive iron doped lithium niobate crystal
}

\author{
Lusine Tsarukyan, Anahit Badalyan, Fabrice Devaux, Mathieu Chauvet, \\ Rafael Drampyan
}

\section{To cite this version:}

Lusine Tsarukyan, Anahit Badalyan, Fabrice Devaux, Mathieu Chauvet, Rafael Drampyan. Pyroelectric-controlled bending of a self-trapped optical beam in a photorefractive iron doped lithium niobate crystal. Journal of Nonlinear Optical Physics \& Materials, 2021, 30 (01n02), pp.1-19. hal03549414

\author{
HAL Id: hal-03549414 \\ https://hal.science/hal-03549414
}

Submitted on 31 Jan 2022

HAL is a multi-disciplinary open access archive for the deposit and dissemination of scientific research documents, whether they are published or not. The documents may come from teaching and research institutions in France or abroad, or from public or private research centers.
L'archive ouverte pluridisciplinaire HAL, est destinée au dépôt et à la diffusion de documents scientifiques de niveau recherche, publiés ou non, émanant des établissements d'enseignement et de recherche français ou étrangers, des laboratoires publics ou privés. 


\title{
Pyroelectric-controlled bending of a self-trapped optical beam in a photorefractive iron doped lithium niobate crystal
}

\author{
Lusine Tsarukyan ${ }^{* \dagger}$, Anahit Badalyan ${ }^{\dagger}$, Fabrice Devaux ${ }^{*}$, \\ Mathieu Chauvet ${ }^{\star}$ and Rafael Drampyan ${ }^{\dagger}$ \\ ${ }^{\dagger}$ Institute for Physical Research, National Academy of Sciences of Armenia, 0203 Ashtarak-2, Armenia \\ *EMTO-ST Institute, University of Bourgogne Franche-Comte, F-25030, Besancon Cedex, France \\ *lustsarukyan@gmail.com
}

We present the experimental demonstration of a self-trapped optical beam bending in a photorefractive $\mathrm{Fe}$ doped lithium niobate (LN:Fe) crystal controlled by the pyroelectric effect. Formation of self-trapped beams with typical $\sim 50 \mu \mathrm{m}$ diameter and large bending of $\sim 140 \mu \mathrm{m}$ are depicted in a $1 \mathrm{~cm}$ length LN:Fe crystal for a laser beam at $632.8 \mathrm{~nm}$ wavelength and $0.5 \mathrm{~mW}$ power with a 30 degree crystal temperature change. The self-trapped beam bending is opposite to the crystal $\mathrm{C}^{+}$-axis. The underlying physics is elaborated and numerical simulations are performed. The long-living waveguiding channels with controlled curvilinear trajectories are promising for optical information routing.

Keywords: Nonlinear optics; Light propagation effects; Self-focusing; Photorefractive lithium niobate crystal; Photovoltaic effect; Pyroelectric effect

\section{Introduction}

Self-trapped optical beams and more specifically spatial optical solitons are the subject of intense studies in nonlinear optics for both their fundamental interest and numerous potential applications ${ }^{1}$, 2. Such self-confined optical beams that can be formed and propagate in specific nonlinear media without change of transverse profile are called spatial solitons. Bright solitons occur when the self-

\footnotetext{
*Corresponding author: lustsarukyan@gmail.com
} 
focusing due to a light-induced positive change of the refractive index balances the linear diffraction. For dark soliton, a dark notch is superimposed on a uniform background illumination. The self-defocusing due to light-induced negative refractive index change balances the diffraction of the notch. The bright and dark solitons have been observed in photorefractive crystals ${ }^{3,4}$, liquid crystals ${ }^{5,6}$ and atomic vapors ${ }^{7}$. The solitons which are formed in nematic liquid crystals were termed nematicons ${ }^{8}$.

Photorefractive spatial solitons can be created by $\mathrm{cw}$ light with power as low as $1 \mu \mathrm{W}$, however, the application of strong external electric fields to the photorefractive crystals is most often necessary ${ }^{9-11}$. Photovoltaic solitons are classified separately because they do not require the applications of external static electric field but rely on the photovoltaic effect.

Among photorefractive crystals the lithium niobate (LN) crystal attracts special attention because of its excellent photorefractive, photovoltaic and pyroelectric properties. The nonlinear properties of LN crystals have been studied by Z-scan technique in numerous works (see, Refs. 12, 13, and references therein). In the absence of applied electric field the light-induced refractive index is negative in LN crystal which is thus appropriate to support the dark solitons. Dark solitons generation in bulk LN crystal due to photovoltaic effect is reported in Refs. 14, 15.

The generation of bright spatial optical solitons in LN crystal is also possible by application of an external static field which is stronger than the photovoltaic field. The screening-photovoltaic bright soliton was observed for the first time in undoped LN crystal with application of a $3.5 \times 10^{6}$ V/m static electric field in Ref. 16.

In Ref. 17 it was proposed to use the pronounced pyroelectric properties of ferroelectric LN crystal instead of a static external electric field to induce large positive optical nonlinearity. The pyroelectric controlled bright soliton was generated in a LN crystal heated 10 degree above initial 
temperature. This spatial soliton was termed pyroliton. Pyroelectric photovoltaic spatial solitons in unbiased photorefractive crystals have been studied further theoretically in Ref. 18 -20.

The interaction between solitons is an intriguing configuration. To date the spiraling, crossing and interlacing of solitons have been predicted and observed ${ }^{20-24}$.

Very interesting for applications is also the possibility of generation of the solitons with curvilinear trajectory. The deflection of bright solitons was simulated for biased strontium barium niobate crystal in Refs. 25, 26 taking into account the charge diffusion process in addition to the photovoltaic drift of the charges which introduces the asymmetry in the induced refractive waveguide. A weak deflection of 10-20 $\mu \mathrm{m}$ in typical $10 \mathrm{~mm}$ long crystal was predicted. A giant self-deflection $(\sim 300 \mu \mathrm{m})$ of $10 \mu \mathrm{m}$ size soliton beam in an undoped $7 \mathrm{~mm}$ long $\mathrm{LN}$ crystal with externally applied static field of $6 \times 10^{6} \mathrm{~V} / \mathrm{m}$ was demonstrated in Refs. 27, 28. The large selfdeflection was attributed to the low concentration of donor and acceptor impurities in the undoped LN crystal that gives a saturation of the induced space-charge field.

In Ref. 29 the phenomenon of micrometric scale 2D regular optical patterns formation by 0.5 $\mathrm{mW}$ power laser beam crossing a photorefractive Fe doped $\mathrm{LN}$ (LN:Fe) crystal was reported. The results were explained by light-induced complex lens structure in the crystal which produces light defocusing from the beam center and focusing on the periphery. Mutual interference of the distorted beams causes the regular optical patterns formation. The regular optical pattern inside the crystal, in its turn, can lead to the formation of quasi-crystalline photonic structure in LN:Fe crystal ${ }^{29,30}$. The first results of modification of the laser beam profile by pyroelectric effect during propagation through LN:Fe crystal were also reported in Ref. 29.

In this paper we report novel results on the generation of pyroelectric controlled bending of a self-trapped optical beam in $\mathrm{LN}: \mathrm{Fe}$ crystal with a $30^{\circ} \mathrm{C}$ increase of the crystal temperature. Experimental results are supported by numerical simulations in good agreement. 


\section{Experimental setup}

The experimental setup is similar to that used in Ref. 30. The laser source is a single mode He-Ne laser at $632.8 \mathrm{~nm}$ wavelength with maximum power of $10 \mathrm{~mW}$. In the experiment a $0.03 \mathrm{wt} \%$ $\mathrm{LN}$ :Fe crystal whose length is $10 \mathrm{~mm}$ along beam propagation $\mathrm{Z}$ is used. Transverse dimensions of the crystal are $4.2 \mathrm{~mm} \times 4.4 \mathrm{~mm}(\mathrm{X} \times \mathrm{Y})$. The optical C-axis of the crystal is oriented in the vertical $Y$ direction. The laser beam power was adjusted by combination of a half-wave plate and a polarizing beam splitter. Both ordinary $(o)$ and extraordinary $(e)$ polarized laser beams relative to the C-axis of the crystal were used in the experiment. The $0.5 \mathrm{~mW}$ power laser beam was focused with a short focal length objective (3.8×) to a spot with full width at half maximum (FWHM) of $\sim 72 \mu \mathrm{m}$ at the input face of the LN:Fe crystal. The crystal is placed between insulating plastic covers inside a metallic mount whose temperature was accurately controlled from the bottom side by Peltier element attached to a controller. The heating device provides a change of the crystal temperature in the range of $10 \div 45^{\circ} \mathrm{C}$ in less than $150 \mathrm{~s}$.

The forward traveling laser beam pattern at the output face of the crystal is imaged by a lens to a CMOS camera. It provides the registration and measurements of the output beam transverse profile evolution. The output light from the crystal is attenuated before CMOS camera to provide its operation in a linear regime.

Both laser beam profiles at the input and output faces of the crystal are also analyzed by a beam profiler system (Thorlabs BP-106-VIS). Beam profiles measured along both X and Y (C-axis of the crystal) directions are well approximated by Gaussian function. The measurements were performed for a very low laser beam power of $20 \mu \mathrm{W}$ to avoid any nonlinear distortion of the beam. The measured FWHMs of the beam along X- and Y-axes at the input and output of the crystal are 72 $\mu \mathrm{m}$ and $124 \mu \mathrm{m}$, respectively. Input beam intensity for $0.5 \mathrm{~mW}$ power is estimated of $\sim 10^{5} \mathrm{~W} / \mathrm{m}^{2}$. 


\section{Motivation of experiment}

$\mathrm{LN}: \mathrm{Fe}$ crystal is well known for its excellent photorefractive and pyroelectric properties. The illumination of the crystal by milliwatt power light leads to the efficient photo-generation of free charges from $\mathrm{Fe}^{2+}$ donor centers to the conduction band. Because of photovoltaic effect, the electrons move along the $\mathrm{C}$-axis of the crystal and recombine in $\mathrm{Fe}^{3+}$ acceptor centers in darker zones of the crystal. The redistribution of the charges leads to the formation of a space-charge electric field $E_{\mathrm{SC}}$ whose amplitude can rise up to the photovoltaic field $E_{\mathrm{PV}}$. The photovoltaic field induces a refractive index changes by electro-optic effect: $\Delta n_{0}=-0.5 n_{0}{ }^{3} r_{13} E_{P V}$ and $\Delta n_{e}=-$ $0.5 n_{e}{ }^{3} r_{33} E_{P V}$, where $n_{0}=2.28, n_{\mathrm{e}}=2.19$ and $r_{13}=9.1 \mathrm{pm} \cdot \mathrm{V}^{-1}$ and $r_{33}=30.9 \mathrm{pm} \cdot \mathrm{V}^{-1}$ are the electrooptic coefficients for $o$ - and $e$-polarized lights, respectively, at $633 \mathrm{~nm}$ wavelength ${ }^{31,32}$. The space charge field due to the photovoltaic effect is determined by the formula $E_{\mathrm{PV}}=k \alpha I / \sigma$, where $I$ is the light intensity, $k$ is a factor depending on the nature of absorbing centers and light wavelength and independent of the impurity concentration, $\alpha$ is the absorption coefficient and $\sigma$ is the photoconductivity of the illuminated part of the crystal ${ }^{33}$. The refractive index change is larger for extraordinary index due to the larger electro-optic coefficient.

Pyroelectric crystal, such as LN, exhibits a variation of spontaneous polarization $P_{\mathrm{s}}$ as a function of temperature change $\Delta T$, given by its pyroelectric coefficient $p=d P_{\mathrm{s}} / d T$, leading to the induction of internal pyroelectric field $E_{\mathrm{PY}}$. The $E_{\mathrm{PY}}$ is given by ${ }^{17}$ :

$$
E_{P Y}=\Delta E=-\left(1 / \varepsilon_{0} \varepsilon_{r}\right) p \Delta T
$$

where $\varepsilon_{0}$ and $\varepsilon_{\mathrm{r}}$ are the vacuum and relative dielectric constants, respectively. For $\mathrm{LN}$ the pyroelectric coefficient equals to $p \approx-6 \times 10^{-5} \mathrm{C} \mathrm{m}^{-2} \mathrm{~K}^{-1}$ at $25^{\circ} \mathrm{C} 34,35$. Thus, in open circuit configuration the internal pyroelectric field reaches $\sim 6 \times 10^{6} \mathrm{~V} / \mathrm{m}$ under temperature increase of $30^{\circ} \mathrm{C}$. This field is oriented along the $\mathrm{C}$-axis of the crystal and can either be positive or negative by 
increase or decrease of the crystal temperature, respectively. The $E_{\mathrm{PY}}$ gives a drift current that opposes to the photovoltaic current. As a consequence, if $E_{\mathrm{PY}}>E_{\mathrm{PV}}$, the net field can be positive and induce the focusing effect in the illuminated region. Thus the pyroelectric effect can strongly influence the propagation features of the optical beam.

\section{Experimental results and discussions}

\subsection{Pyroelectric controlled beam self-localization and deflection}

The self-patterning of a $o$-polarized single Gaussian beam of $632.8 \mathrm{~nm}$ wavelength and $0.5 \mathrm{~mW}$ power during propagation through a $10 \mathrm{~mm}$ length LN:Fe crystal was demonstrated in Refs. 29, 30. The crystal was kept at room temperature of $20^{\circ} \mathrm{C}$. The milliwatt level power of the optical beam provided a slow evolution of nonlinear processes in the crystal. It allowed an easy observation of the optical patterns formation dynamics. Figure 1 shows examples of the regular 2D optical patterns temporal evolution at the exit of the crystal.

In the present study the optical patterns temporal evolution is investigated under pyroelectric effect by gradual heating of the crystal in the temperature range of $10-42{ }^{\circ} \mathrm{C}$ (Figure 2 ).

The observations for a $o$-polarized laser beam are shown in Figure 3. The beam evolution demonstrated in Figure 3 can be understood as follows. In the time interval of 1-10 s the crystal temperature increase is small and the photovoltaic effect still plays a dominant role. The refractive index decreases in the beam centre and leads to the beam splitting into two symmetric lobes, because the light tends to deflect to areas with larger refractive index on the beam periphery (Figure 3(b)). Separation distance between the lateral lobes increases over the time. As the pyroelectric field gradually increases and condition $E_{\mathrm{PY}}>E_{\mathrm{PV}}$ is satisfied, the space charge field changes sign and induces positive refractive index modulation and focusing effect in the illuminated region. The light power begins to concentrate in the lower light spot (Figures 3(b)-(e)) as a result of some asymmetry in the modified refractive index distribution, which physical origin will be discussed in Sec. 4.2. 
Maximum power concentration is observed at 39-45 s (Figures 3(d)-(e)). The transverse size of the localized beam is approximately twice less than that of the initial output beam (Figure 3(a)).

At time interval of $125-200 \mathrm{~s}$ of the process evolution there is no temperature change $\Delta T$ (Figure 2) and no pyroelectric field generation (Eq. (1)). Moreover, the light erases the pyroelectric space-charge field generated at the early stage and the photovoltaic effect again becomes dominant. The erasure time of the space-charge field by light of milliwatt power is a few minutes ${ }^{36,37}$. This leads to the beam profile evolution (Figures $3(\mathrm{~g}),(\mathrm{h})$ ) similar to the observation without pyroelectric effect for the crystal constant temperature of $20^{\circ} \mathrm{C}$ (see Figures $1(\mathrm{c}),(\mathrm{d})$ ).

The interaction of laser beam with $\mathrm{LN}$ crystal can be stronger with the use of a $e$-polarized beam because of the larger electro-optic coefficient $r_{33}$. The use of a $e$-polarized beam with same power of $0.5 \mathrm{~mW}$ is expected to speed the process and provide more pronounced beam selflocalization and deflection.

Pyroelectric controlled self-trapping for $e$-polarized laser beam is studied for the crystal temperature increase regime. Figure 4 shows the measured temperature increase of the crystal in the performed experiment. Experimental results for the time evolution of the profile of $e$-polarized, $632.8 \mathrm{~nm}$ beam of a $0.5 \mathrm{~mW}$ power passed through the $10 \mathrm{~mm}$ thick $\mathrm{LN}: F e$ crystal when the crystal temperature is increased from 10 to $45^{\circ} \mathrm{C}$ are shown in Figure 5 . The beam size along $\mathrm{C}$-axis at the output face of the crystal reduces from $120 \mu \mathrm{m}$ at instant $t=0$ to $53 \mu \mathrm{m}$ at $t=27 \mathrm{~s}$. The localized beam intensity shows $\sim 2.5$ times increase.

More detailed measurements of beam profile modification and self-focusing, as well as soliton deflection for crystal heating are depicted in Figures 6 and 7. The graphs in Figure 6 show the beam FWHM narrowing and intensity increase with time along X (Figures 6(a), (b)) and Y (Caxis of the crystal) (Figures 6(c), (d)) transverse directions. The increase of the beam FWHM in the time interval of $0-5 \mathrm{~s}$ in (c) is connected with the stretching of the optical beam along crystal C-axis. 
The total beam splitting takes place at time instant of $7 \mathrm{~s}$. Subsequent measurements of the beam FWHM were performed for the lower lobe. The intensity decrease at $t=7 \mathrm{~s}$ in (b) and (d) is connected with beam splitting along $\mathrm{C}$-axis of the crystal. The circles and triangles show, respectively, the beam FWHM and intensity. The larger size circles and triangles depict the initial values.

Figure 7 demonstrates the time evolution of a self-trapped optical beam deflection opposite to the $\mathrm{C}^{+}$-axis as illustrated in Figures 5(a)-(e). Measurements are performed relative to the input beam center. The maximum deflection of $\sim 140 \mu \mathrm{m}$, equaled roughly to three soliton's diameter of $\sim 53 \mu \mathrm{m}$, is observed experimentally.

\subsection{Physical model}

The beam self-focusing and bending opposite to the crystal $\mathrm{C}^{+}$-axis in the undoped $\mathrm{LN}$ crystal for applied static electric field of $E_{0}=5 \times 10^{6} \mathrm{~V} / \mathrm{m}$, light intensity $I \sim 10^{3} \mathrm{~W} / \mathrm{m}^{2}$, acceptors concentration $N_{\mathrm{A}}=2 \times 10^{21} \mathrm{~m}^{-3}$ and $N_{\mathrm{D}} / N_{\mathrm{A}}=1.01$, where $N_{\mathrm{D}}$ is the concentration of donors, was demonstrated in Refs. 27, 28. The static electric field $E_{0}$ was applied along the crystal $\mathrm{C}^{+}$-axis in order to counteract the photovoltaic induced self-defocusing and obtain the self-focusing effect.

In our experiment we used LN:Fe crystal with larger concentration of donors and pyroelectric field instead of static electric field. When the crystal is heated the pyroelectric field gradually increases. The photovoltaic field induced beam splitting occurs at early stage of the process when the condition $E_{\mathrm{PY}}<E_{\mathrm{PV}}$ is satisfied (Figures 3(b) and 5(b)). Further gradual increase of the pyroelectric field with crystal temperature raise leads to the condition $E_{\mathrm{PY}}>E_{\mathrm{PV}}$. The $E_{\mathrm{PY}}$ gives a drift current that is opposite to the photovoltaic current. The resulting space-charge field changes sign and induces a positive refractive index modulation in the illuminated region and the focusing effect takes place (Figures 3(c)-(e) and 5(c)-(e)). 
The spontaneous polarization change by the temperature increase of the crystal (Figures 2 and 4) is the main mechanism for the suppression of the defocusing in LN:Fe crystal giving rise of the self-focusing effect. However, the spatial temperature gradient along crystal C-axis ${ }^{38}$ also should be taken into account. According to Ref. 38 the spatial gradient of temperature $\nabla T \sim 1.2 \times 10^{3}$ ${ }^{\circ} \mathrm{C} / \mathrm{m}$ induces $\sim 10^{5} \mathrm{~V} / \mathrm{m}$ pyroelectric field which provides the suppression of photorefractive damage in the undoped LN crystal. However, for the present experiment conditions the pyroelectric field due to the spatial temperature gradient is one order of magnitude lower than the estimated value $\sim 10^{6}$ $\mathrm{V} / \mathrm{m}$ of pyroelectric field, induced by heating of the crystal to $45^{\circ} \mathrm{C}$ (Eq. (1)).

In the current experiment the crystal was heated in the range of $10-45^{\circ} \mathrm{C}$ from the bottom side $(-\mathrm{C}$ pole), hence the upper side of the crystal $(+\mathrm{C}$ pole) has relatively lower temperature. The temperature difference of $4-5^{\circ} \mathrm{C}$ will provide the spatial gradient of $10^{3}{ }^{\circ} \mathrm{C} / \mathrm{m}$ for $4.4 \mathrm{~mm}$ thick crystal along $\mathrm{C}$-axis. While this value is not enough to suppress the optical damage in $\mathrm{LN}: \mathrm{Fe}$ crystal, it can induce an asymmetry in the resulting refractive index distribution.

Higher temperature and its larger change $\Delta T$ at $-\mathrm{C}$ side of the crystal can provide a larger pyroelectric field (Eq. (1)) and its asymmetric distribution along C-axis of the crystal. Similar distribution will have pyroelectric field induced refractive index variation shown in Figure 8(a). Figure 8(a) shows also the refractive index variation due to the photovoltaic field that has deep central minimum $\Delta n<0$ and side maxima $\Delta n>0$ along crystal $\mathrm{C}^{+}$-axis ${ }^{12,31}$. Since $E_{\mathrm{PY}}$ is directed opposite to $E_{\mathrm{PV}}$, hence for $E_{\mathrm{PY}}>E_{\mathrm{PV}}$ the resulting pyroelectric field induced refractive index change will have a positive sign. Its maximum is shifted relative to the beam center towards the $-\mathrm{C}$ pole of the crystal, which is schematically shown in Figure 8(b).

The analysis performed shows that the combined action of photovoltaic and pyroelectric fields can thus lead to a refractive index asymmetric profile formation with a peak shifted opposite to the crystal $\mathrm{C}^{+}$-axis. The obtained asymmetry in refractive index distribution will dictate the self- 
localized light displacement direction that is opposite to the crystal $\mathrm{C}^{+}$-axis. The experimentally obtained optical beam self-localization in the lower light spot of the split beam is due to a selffocused light deviation opposite to the crystal $\mathrm{C}^{+}$-axis and is clearly seen in Figure 5(a)-(e). The magnitude of the beam displacement over time is shown in Figure 7.

\section{Numerical simulations}

Numerical simulations of the beam propagation through the LN crystal were performed. Note that a beam with larger intensity $\geq 10^{5} \mathrm{~W} / \mathrm{m}^{2}$ and a doped $\mathrm{LN}$ :Fe crystal have been used in our experiment compared to the studies reported in Refs. 27,28 , where nominally pure LN crystals with relatively low concentrations of donors were utilized. Simulations performed with a single deep center model 39 were first realized but failed to explain the experimentally observed behavior. Thus, a more elaborated two-photoactive-center model for LN:Fe crystal ${ }^{32,40,41}$ where iron ions and polarons are both considered as the photoactive centers has been used. Polaron is a quasiparticle which is selflocalized in a single lattice site and moves in the crystal by thermal or light-induced hopping among different sites $32,41-43$.

Different types of small electron polarons are distinguished in $\mathrm{LN}$ crystal ${ }^{43}$. Free $\mathrm{Nb}_{\mathrm{Nb}}{ }^{4+}$ small polarons localized in regular $\mathrm{Nb}$ site, small polaron bound to antisite defects $\mathrm{Nb}_{\mathrm{Li}}{ }^{4+}$ and bound small $\mathrm{Nb}_{\mathrm{Li}}{ }^{4+}: \mathrm{Nb}_{\mathrm{Nb}}{ }^{4+}$ bipolarons. In $\mathrm{LN}: \mathrm{Fe}$ crystal the dominant photoactive center has been identified as $\mathrm{Nb}_{\mathrm{Li}}$ polarons. Charge redistribution takes place by hopping of electrons between intrinsic defect centers $\mathrm{Nb}_{\mathrm{Li}}{ }^{4+/ 5+}:\left(\mathrm{Nb}^{4+} \leftrightarrow \mathrm{Nb}^{5+}+\mathrm{e}^{-}\right)^{32,41}$. The concentration of bound polarons $N_{\mathrm{X} 0}$ is usually assumed to be greater than $10^{26} \mathrm{~m}^{-3}{ }^{32}$. Additional $\mathrm{Nb}_{\mathrm{Li}}{ }^{4+}$ polarons can be generated due to dissociation of bipolarons $\mathrm{Nb}_{\mathrm{Li}}{ }^{4+}: \mathrm{Nb}_{\mathrm{Nb}}{ }^{4+}$ under intense light illumination ${ }^{13,43}$.

The charge-transport mechanism, based on small polarons hopping, can play an essential role in the photorefractive properties of $\mathrm{LN}: \mathrm{Fe}$ crystal. The $\mathrm{Fe}^{2+} / \mathrm{Fe}^{3+}$ charge-transport model modified 
by additional polaron X-centers is described in details in Ref. 32. Simulations performed in Ref. 30 based on two-photoactive center model shows that the light induced specific refractive index change $\Delta n$ in LN:Fe crystal with negative central deep and two positive side maxima has a temporal evolution with tendency of reducing the central minimum and increasing the positive side maxima. This tendency provides the favorable conditions for compensation of negative refractive index change by pyroelectric effect and induction of positive refractive index change in the illuminated areas and thus the focusing effect. The following two-photoactive- center model is applied for simulations of self-focusing in LN:Fe crystal under pyroelectric effect:

$$
\begin{gathered}
\frac{\partial N_{F e}^{+}}{\partial t}=\left(s_{F e}+s_{F e X} N_{X}^{+}\right) I\left(N_{F e}-N_{F e}^{+}\right)-\left[\gamma_{F e} N_{e}+\gamma_{X F e}\left(N_{X}-N_{X}^{+}\right)\right] N_{F e}^{+} \\
\frac{\partial N_{X}^{+}}{\partial t}=\left(s_{X} I+\gamma_{X F e} N_{F e}^{+}\right)\left(N_{X}-N_{X}^{+}\right)-\left[\gamma_{X} N_{e}+s_{F e X} I\left(N_{F e}-N_{F e}^{+}\right)\right] N_{X}^{+} \\
\frac{\partial N_{e}}{\partial t}=\frac{\partial N_{F e}^{+}}{\partial t}+\frac{\partial N_{X}^{+}}{\partial t}-\frac{1}{e} \frac{\partial \rho}{\partial t} \\
\vec{J}=e \mu N_{e} \vec{E}+k_{F e} I\left(N_{F e}-N_{F e}^{+}\right) \vec{c}+k_{X} I\left(N_{X}-N_{X}^{+}\right) \vec{c}+\mu k_{B} T \vec{\nabla} N_{e} \\
\frac{\partial \rho}{\partial t}=-\vec{\nabla} \cdot \vec{J}
\end{gathered}
$$

where $\mathrm{Ne}, \mathrm{N}^{+} \mathrm{Fe}$, and $N^{+} \mathrm{X}$ are, respectively, the densities of free electrons, ionized iron, and ionized polarons. $I$ is the light intensity, $s_{\mathrm{Fe}}, s_{X}$, and $s_{\mathrm{Fe} X}$ are the photoionization cross sections of electrons from, respectively, the $\mathrm{Fe}^{2+}$ and $X$ states into the conduction band and from $\mathrm{Fe}^{2+}$ to $X^{+}$states. $\gamma_{\mathrm{Fe}}, \gamma_{X}$, and $\gamma_{X \mathrm{Fe}}$ are the corresponding recombination rates of electrons. Equation (2d) is the expression of the total current density $J$ where drift current, photovoltaic (PV) currents of both photoactive centers, and thermal diffusion are considered. $\kappa_{\mathrm{Fe}}$ and $\kappa_{X}$ are the PV coefficients. $\vec{E}$ represents the total electric field in the material, including the pyroelectric field component $E_{\mathrm{py}}$ which is oriented along the crystal c-axis. $E_{\mathrm{py}}$ is calculated using equation (1). The space-charge density is $\rho=e\left(N^{+} \mathrm{Fe}+\right.$ 
$N^{+}{ }^{-} N_{A}-N_{e}$ ), where the constant acceptor concentration $N_{A}$ corresponds to the shallow acceptor density. $e$ and $\mu$ are, respectively, the elementary charge and the mobility of electrons. $k_{B}$ is the Boltzmann constant, and $T$ is the temperature.

This system of equations (2) that describe the charges generation, displacement and recombination are solved to deduce the densities of free electrons, ionized iron and polarons ${ }^{41}$ which, in turn, allow the simulation of the space charge field and the induced refractive index change distribution in the crystal. The modification of laser beam intensity distribution during propagation through the crystal is also obtained. Our model and resolution method are derived from Ref. 41.

The following main experimental parameters were used for simulations: the laser beam wavelength is $632.8 \mathrm{~nm}$, the beam intensity is $\geq 10^{5} \mathrm{~W} / \mathrm{m}^{2}$, the polarization of beam is extraordinary, the crystal length is $10 \mathrm{~mm}$ and the concentration of Fe ions in the crystal is $0.03 \mathrm{wt} \%$. Both spatial and temporal gradients of the crystal temperature for heating from $T_{0}=10^{\circ} \mathrm{C}$ to $T_{\mathrm{p}}=45^{\circ} \mathrm{C}$ during $135 \mathrm{~s}$ (Figure 4) were taken into account. Since the crystal was heated from the bottom side which is in contact with the Peltier element, we consider a temperature difference $\Delta T \sim 5-10{ }^{\circ} \mathrm{C}$ between the crystal bottom and upper faces. The spatial gradient of temperature along crystal C-axis can be estimated by $\Delta T / d$ where $d=4.4 \mathrm{~mm}$ is crystal transverse size along $\mathrm{Y}$ direction ${ }^{38}$. Thus, $\Delta T / d \sim$ $-10^{3}{ }^{\circ} \mathrm{C} / \mathrm{m}$. The heating temperature rise versus time $t$ we approximated by the formula:

$$
T(t)=T_{0}+\left(T_{p}-T_{0}\right) \cdot\left(\frac{t / \tau}{1+(t / \tau)}+2 \cdot \frac{t / \tau}{[1+(t / \tau)]^{2}}\right)
$$

where $\tau$ is the time to reach the peak temperature. The deduced $T(t)$ dependence provides the evaluation of the temperature up the plateau at the temperature $T_{\mathrm{p}}=45^{\circ} \mathrm{C}$ corresponding to the experimental conditions. According to the experimental results (Figures 5,6) we take $\tau=30 \mathrm{~s}$ in the simulations to provide the temperature gradient. 
Other parameters of LN:Fe crystal taken from Refs. 32, 41 are given in Table 1.

Table 1: Parameters for LN:Fe crystal involved in the numerical model.

\begin{tabular}{|c|c|c|}
\hline $\begin{array}{l}\text { Parameter } \\
(\lambda=633 \mathrm{~nm})\end{array}$ & Meaning & Value \\
\hline$\mu\left(\mathrm{m}^{2} \mathrm{~V}^{-1} \mathrm{~s}^{-1}\right)$ & Mobility of electric charges & $7.4 \times 10^{-5}$ \\
\hline$S_{F e}\left(\mathbf{m}^{2} \mathbf{J}^{-1}\right)$ & Cross-section of photoionization of donor & $3.3 \times 10^{-6}$ \\
\hline$S_{X}$ & Cross-section of photoionization of polarons & $5 \times 10^{-5}$ \\
\hline$S_{F e X}\left(\mathbf{m}^{5} \mathbf{J}^{-1}\right)$ & $\begin{array}{l}\text { Coupling efficiency between } \\
\text { polarons-donors }\end{array}$ & $3.22 \times 10^{-30}$ \\
\hline$\gamma_{F e}\left(\mathrm{~m}^{3} \mathrm{~s}^{-1}\right)$ & Recombination rate of electrons from $\mathrm{Fe}^{2+}$ & $1.65 \times 10^{-14}$ \\
\hline$\gamma_{X}$ & $\begin{array}{l}\text { Recombination rate of electrons } \\
\text { from } \mathrm{X}^{+} \text {-states }\end{array}$ & $5 \times 10^{-16}$ \\
\hline$\gamma_{X F e}$ & Recombination rate from $\mathrm{X}^{+}$states to $\mathrm{Fe}^{3+}$ & $1.14 \times 10^{-21}$ \\
\hline$K_{F e}^{o}\left(m^{3} V^{-1}\right)$ & $\begin{array}{l}\text { Photovoltaic coefficient of donors for } \\
o \text {-polarized light }\end{array}$ & $-3.5 \times 10^{-33}$ \\
\hline$K_{F e}{ }^{e}$ & $\begin{array}{l}\text { Photovoltaic coefficient of donors for } \\
e \text {-polarized light }\end{array}$ & $-7 \times 10^{-33}$ \\
\hline$K_{X}^{o}$ & $\begin{array}{l}\text { Photovoltaic coefficient of polarons for } \\
o \text {-polarized light }\end{array}$ & $-21.2 \times 10^{-33}$ \\
\hline$K_{X}{ }^{e}$ & $\begin{array}{l}\text { Photovoltaic coefficient of polarons for } \\
e \text {-polarized light }\end{array}$ & $-42.4 \times 10^{-33}$ \\
\hline Fr._Fe & Fraction of ionized donors & 0.9 \\
\hline Fr. $X$ & Fraction of ionized polarons & 1.0 \\
\hline
\end{tabular}

The parameters correspond to LN:Fe crystal for optical beam intensities $I \geq 10^{5} \mathrm{~W} / \mathrm{m}^{2}$. Since deep levels concentrations are not accurately known we took the densities of ionized iron donors $N_{\mathrm{d} 0}$ and polaron centers $N_{\mathrm{X} 0}$, as free parameters. It is worth to note that while the input beam power is $\sim 0.5 \mathrm{~mW}\left(I \sim 10^{5} \mathrm{~W} / \mathrm{m}^{2}\right)$ in the experiment, we considered the beam power also as a free parameter in the range of $0.5-10 \mathrm{~mW}$. The good coincidence of experiment and simulations was obtained for 10 $\mathrm{mW}$ beam power $\left(I \sim 2.5 \times 10^{6} \mathrm{~W} / \mathrm{m}^{2}\right)$. The taking of higher intensities in simulations is reasonable because of the beam self-focusing and break-up into much narrower micrometric scale filaments inside the LN crystal that provides one order higher light intensity $I \geq 10^{6} \mathrm{~W} / \mathrm{m}^{2}$ in the medium. 
The higher light intensity, in its turn, increases the contribution of polaron mechanism in photorefractive process. The criterion for selecting the values of $N_{\mathrm{d} 0}$ and $N_{\mathrm{X} 0}$, was the best coincidence with experimental results. Good coincidence with experimental results was obtained for $N_{\mathrm{d} 0}=1.8 \times 10^{25} \mathrm{~m}^{-3}$ and $N_{\mathrm{X} 0}=2 \times 10^{28} \mathrm{~m}^{-3}$.

Figures 9 and 10 show, respectively, the simulated refractive index change $\Delta n$, the beam intensity evolution along propagation in the crystal, as well as the intensity profile in the transverse $\mathrm{X}-\mathrm{Y}$ plane and its distribution along Y (C-axis) direction at two instants of $t=35 \mathrm{~s}$ and $t=80 \mathrm{~s}$.

At an early stage of the process $(t=35 \mathrm{~s})$ the photovoltaic effect is prevalent and the condition $E_{\mathrm{PY}}<E_{\mathrm{PV}}$ is thus satisfied. Figures 9 (c)-(e) show that the beam splits in two equal power fragments symmetrically disposed relative to the input beam centre along C-axis. Separation distance between the lobes increases over time. The simulation correctly describes the experimental results (Figures 3(b), 5(b)).

In the advanced stage of the process the increase of the pyroelectric field due to higher crystal temperature leads to the condition $E_{\mathrm{PY}}>E_{\mathrm{PV}}$. It allows a positive refractive index change in the illuminated areas and thus the focusing effect. Simulations of the laser beam profile evolution between 35-80 s demonstrate the self-focusing of each lobe. Numerical results at $t=80 \mathrm{~s}$ are shown in Figure 10. The lobe localized at the $-\mathrm{C}$ pole of the crystal demonstrates the narrowing and bending toward $\mathrm{C}^{-}$-axis while the lobe positioned closer to the $+\mathrm{C}$ face of crystal shows a spreading and an intensity decrease (Figures 10(b)-(d)). Thus, the simulations show the gradual deflection of the formed self-trapped optical beam opposite to the crystal $\mathrm{C}^{+}$-axis in accordance with the experimental results demonstrated in Figure 5 and to physical model described in Sec.4.2.

At a simulation time of $80 \mathrm{~s}$ (Figures 10(b)-(d)) the self-trapped beam formation and its maximum bending correspond to the experimental time of $30 \mathrm{~s}$ for this process development (see Figures 5(e), 6(c) and 7). We may infer that the theoretical model cannot take into account all 
nuances of the experiment.

Figure 11 shows the simulated time dependence of self-localized beam deflection $\Delta \mathrm{Y}_{\mathrm{sml}}$ relative to the input beam center. The simulated results give qualitatively correct description of the experimentally measured increase of soliton self-deflection versus time, depicted in Figure 5 and in graphs of Figure 7. In Figure 11 the deflection linearly increases with time in the simulation range of 0-60s with tendency of the saturation for further evolution, in accordance with experimental results.

Thus the most features of the observed phenomena of the pyroelectric controlled deflection of the self-focused beam are correctly described by the two-photoactive-center model.

This model provides also the better understanding of the underlying physics.

\section{Conclusion}

In conclusion the complex behavior of a self-trapped beam in a photorefractive LN:Fe crystal, controlled by the pyroelectric effect, is observed and explained. The formation of a pyroelectric controlled self-trapped beam with a $\sim 50 \mu \mathrm{m}$ diameter accompanied with a large bending of $\sim 140 \mu \mathrm{m}$ were observed experimentally for a $e$-polarized, $0.5 \mathrm{~mW}$ beam triggered by a moderate 30 degree crystal temperature increase. The self-focusing effect is attributed to the crystal heating from 10 to $45^{\circ} \mathrm{C}$ resulting in positive refractive index variation.

The self-trapped beam bending opposite to the crystal $\mathrm{C}^{+}$-axis is explained by a spatial gradient of temperature along crystal $\mathrm{C}$-axis with larger temperature near $-\mathrm{C}$ pole of the crystal. The asymmetry in the resulting refractive index variation along $\mathrm{C}$-axis, with a maximum of refractive index shifted towards the $-\mathrm{C}$ pole of the crystal, dictates the self-trapped beam displacement opposite to the crystal $\mathrm{C}^{+}$- axis. 
The numerical simulations were performed based on a two-photoactive-center model for LN:Fe crystal, where iron ions and polarons are both considered as photoactive centers. The simulation results are in good agreement with obtained experimental results.

The generated self-trapped beams create waveguiding channels in the photorefractive crystal which are long-living (few months of dark decay) due to the high resistivity of LN:Fe crystal. The long dark-storage time soliton waveguide in undoped LN crystal was reported before in Ref. 44. The long-living waveguiding channels with pyroelecrtric controlled curvilinear trajectory are promising for optical information routing.

\section{Acknowledgements}

This work was supported by International Science and Technology Center (ISTC) [Project A-2130]. The authors are grateful to Dr. Edvard Kokanyan for providing the LN:Fe crystals in the framework ISTC A-2130 Project. The authors appreciate Dr. Emil Gazazyan for computational assistance in the framework of ISTC Project A-2130 and Dr. Ruben Hovsepyan for discussions.

\section{References:}

1. S. Trillo, W. E. Torruellas, Spatial solitons (Springer-Verlag, Berlin, 2001).

2. Yu. S. Kivshar, G. P. Agrawal, Optical Solitons (Academic Press: New York, 2003).

3. D. N. Christodoulides, M. I. Carvalho, Bright, dark and gray spatial soliton states in photorefractive media, J. Opt. Soc. Am. B 12 (1995) 1628-1633.

4. A. D. Boardman, A. P. Sukhorukov, Soliton Driven Photonics (Kluver Acad. Publ. Dordrecht, 2001).

5. M. Peccianti, A. De Rossi, G. Assanto, A. De Luca, C. P. Umeton, I. C. Khoo, Electrically assisted self-confinement and waveguiding in planar nematic liquid crystal cells, Appl. Phys. Lett. 77 (2000) 7-9. 
6. G. Assanto, M. Peccianti, Spatial solitons in nematic liquid crystals, IEEE J. Quant. Electron. 39(1) (2003) 13-21.

7. P. Zerom, R. W. Boyd, Self-focusing, Conical Emission, and Other Self-Action Effects in Atomic Vapors (In: R.W. Boyd., S.G. Lukishova, Y. Shen (Eds), Self-focusing: Past and Present. Topics in Applied Physics, Vol. 114, Springer, New York, NY, 2009).

8. A. Piccardi, A. Alberucci, G. Assanto, Nematicons and their electro-optic control: Light localization and signal readdressing via reorientation in liquid crystals, Int. J. Mol. Sci. 14 (2013) 19932-19950.

9. J. Safioui, M. Chauvet, F. Devaux, V. Coda, F. Pettazzi, M Alonzo, E. Fazio, Polarization and configuration dependence of beam self-focusing in photorefractive $\mathrm{LiNbO}_{3}, J$. Opt. Soc. Am. B 26(3) (2009) 487-492.

10. F. Diebel, M. Boguslawski, T. Dadalyan, R. Drampyan, C. Denz, Controlled soliton formation in tailored Bessel photonic lattices, Opt. Express 24 (2016) 12933-12940.

11. S. T. Popescu, A. Petris, V. I. Vlad, Experimental investigation of the output mode profile of soliton waveguides recorded at $405 \mathrm{~nm}$ wavelength in lithium niobate, Rom. Rep. Phys. 63 (2013) 915-924.

12. Q. Wang Song, Ch.-P. Zhang, P. J. Talbot, Self-defocusing, self-focusing, and speckle in $\mathrm{LiNbO}_{3}$ and $\mathrm{LiNbO}_{3}: \mathrm{Fe}$ crystals, Appl. Opt. 32(35) (1993) 7266-7271.

13. S. M. Kostritskii, M. Aillerie, Z-scan study of nonlinear absorption in reduced $\mathrm{LiNbO}_{3}$ crystal, J. Appl. Phys. 111 (2012) 103504 -1-6.

14. M. Taya, M. C. Bashaw, M. M. Fejer, M. Segev, G. C. Valley, Observation of dark photovoltaic spatial solitons, Phys. Rev. A 52 (1995) 3095-3100.

15. M. Chauvet, Temporal analysis of open-circuit dark photovoltaic spatial solitons, J. Opt. Soc. Am. B 20(12) (2003) 2515-2522. 
16. E. Fazio, F. Renzi, R. Rinaldi, M. Bertolotti, M. Chauvet, W. Ramadan, A. Petris, V. I. Vlad, Screening-photovoltaic bright soliton in lithium niobate and associated single-mode waveguides, Appl. Phys. Lett. 85(12) (2004) 2193-2195.

17. J. Safioui, F. Devaux, M. Chauvet, Pyroliton: pyroelectric spatial soliton, Opt. Express 17(24) (2009) 22209-22216.

18. Q. Jiang, Y. Su, X. Ji, Pyroelectric photovoltaic spatial solitons in unbiased photorefractive crystals. Phys. Lett. A 376 (2012) 3085-3087.

19. A. Katti, R. A. Yadav, Spatial solitons in biased photovoltaic photorefractive materials with the pyroelectric effect, Phys. Lett. A 381 (2017) 166-170.

20. A. Katti, Gaussian soliton pairs in an unbiased photorefractive crystal due to the pyroelectric effect, The European Physical Journal Plus 134 (2019) 621- 1-11.

21. W. Krolikowski, B. Luther-Davies, C. Denz, J. Petter, C. Weilnau, A. Stepken, M. Belic, Interaction of two-dimensional spatial incoherent solitons in photorefractive medium, Appl. Phys. B 68 (1999) 975-982.

22. Ya. V. Izdebskaya, J, Rebling, A. S. Desyatnikov, G. Assanto, Yu. S. Kivshar, All-optical switching of a signal by a pair of interacting nematicons, Opt. Express 20 (2012) 2470124707.

23. M. Peccianti, K.A. Brzdakiewicz, G. Assanto, Nonlocal spatial soliton interactions in nematic liquid crystal, Opt. Lett. 27 (2002) 1460-1462.

24. M. Peccianti, C. Conti, G. Assanto, A. De Luca, C. Umeton, Routing of anisotropic spatial solitons and modulational instability in liquid crystals, Nature 432 (2004) 733-737.

25. S. R. Singh. D. N. Christodoulides, Evolution of spatial optical solitons in biased photorefractive media under steady-state conditions, Opt. Commun. 118 (1995) 569-576.

26. M. I. Carvalho, S. R. Singh, D. N. Christodoulides, Self-deflection of steady-state bright spatial soliton in biased photorefractive crystal, Opt. Commun. 120 (1995) 311-315. 
27. M. Chauvet, V. Coda, H. Maillotte, E. Fazio, G. Salamo, Large self-deflection of soliton beams in $\mathrm{LiNbO}_{3}$, Opt. Lett. 30(15) (2005) 1977-1979.

28. F. Devaux, V. Coda, M. Chauvet, R. Passier, New time-dependent photorefractive threedimensional model: application to self-trapped beam with large bending, J. Opt. Soc. Am. B 25 (2008) 1081-1086.

29. L. M. Tsarukyan, A. M. Badalyan, R. K. Hovsepyan, R. Kh. Drampyan, Regular optical patterns formation and pyroelectric controlled beam self-localization in a photorefractive $\mathrm{LiNbO}_{3}:$ Fe crystal, Proc. SPIE 11030 (2019) 1103017 -1-10.

30. L. Tsarukyan, A. Badalyan, F. Devaux, M. Chauvet, R. Drampyan, Generation of regular optical patterns and photonic structures by a single Gaussian beam in a photorefractive $\mathrm{LiNbO}_{3}: \mathrm{Fe}$ crystal, Opt. Commun. 478 (2021) 126396-1-6.

31. F. S. Chen, Optically induced change of refractive index in $\mathrm{LiNbO}_{3}$ and $\mathrm{LiTaO}_{3}, J$. Appl. Phys. 40 (1969) 3389-3396.

32. F. Jermann, J. Otten, Light induced charge transport in $\mathrm{LiNbO}_{3}: \mathrm{Fe}$ at high light intensities, J. Opt. Soc. Am. B 10 (1993) 2085-2092.

33. A. M. Glass, D. von der Linde, T. J. Negran, High-voltage bulk photovoltaic effect in the photorefractive process in $\mathrm{LiNbO}_{3}$, Appl. Phys. Lett. 25 (1974) 233-235.

34. T. Bartholomous, K. Buse, E. Kratzig, Pyroelectric coefficients of $\mathrm{LiNbO}_{3}$ crystals of different compositions, Phys. Status Solidi (a) 142 (1994) K55-K57.

35. M. R. R. Gesualdi, C. Jacinto, T. Catunda, M. Muramatsu, V. Pilla, Thermal lens spectrometry in pyroelectric lithium niobate crystal, Appl. Phys. B 93 (2008) 879-883.

36. K.G. Belabaev, V.B. Markov, S.G. Odulov, Optical erasure of holograms in crystals of lithium niobate, Ukrainian Phys. Journal 21(9) (1976) 142-146. 
37. L. Tsarukyan, R. Hovsepyan, R. Drampyan, Polaron approach for nondestructive readout of holograms in photorefractive $\mathrm{LiNbO}_{3}: \mathrm{Fe}$ crystal in an external magnetic field, Photonics and Nanostructures - Fundamentals and Applications 40 (2020) 100793- 1-16.

38. S. M. Kostritskii, O. G. Sevostyanov, M. Aillerie, P. Bourson, Suppression of photorefractive damage with aid of steady-state temperature gradient in nominally pure $\mathrm{LiNbO}_{3}$ crystal, J. Appl. Phys. 104 (2008) 114104 -1-11.

39. K. Buse, Light induced charge transport processes in photorefractive crystals I: Models and experimental methods, Appl. Phys. B 64 (1997) 273-291.

40. J. Safioui, F. Devaux, K. Ph. Huy, M. Chauvet, High intensity behavior of pyroelectric selffocusing in $\mathrm{LiNbO}_{3}$, Opt. Commun. 294 (2013) 294-298.

41. F. Devaux, J. Safioui, M. Chauvet, R. Passier, Two-photoactive-center model applied to photorefractive self-focusing in biased $\mathrm{LiNbO}_{3}$, Phys. Rev. A 81 (2010) 013825 -1-6.

42. L. Vitadello, M. Bazzan, S. Messerschmidt, M. Imlau, Small polarons hopping in $\mathrm{LiNbO}_{3}$ as a function of temperature and composition, Crystals 8 (2018) 294 -1-13.

43. M. Imlau, H. Badorreck, Ch. Merschjann, Optical nonlinearities of small polarons in lithium niobate, Appl. Phys. Rev. 2 (2015) 040606 -1-26.

44. S. T. Popescu, A. Petris, V. I. Vlad, Fast writing of soliton waveguides in lithium niobate using low-intensity blue light, Appl. Phys. B 108 (2012) 799-805. 


\section{CAPTIONS TO THE FIGURES}

Fig. 1. Time evolution of the $0.5 \mathrm{~mW}$ power, $o$-polarized laser beam profile passed through a 10 mm thick LN:Fe crystal at stable temperature of $20^{\circ} \mathrm{C}$.

Fig. 2. LN:Fe crystal temperature increase during the time with the use of Peltier element. The temperature was measured by measuring the voltage from the calibrated thermocouple which was in contact with the crystal near its center along the vertical Y direction.

Fig. 3. Time evolution of the profile of $o$ - polarized laser beam at $632.8 \mathrm{~nm}$ and power of $0.5 \mathrm{~mW}$ passed through LN:Fe crystal with $10 \mathrm{~mm}$ thickness under pyroelectric effect. The scale shown in (a) is valid for all figures.

Fig. 4. LN:Fe crystal temperature increase during the time with the use of Peltier element. The temperature was measured by measuring the voltage from the calibrated thermocouple which was in contact with the crystal.

Fig. 5. (a-e) Time evolution of $e$ - polarized laser beam at $632.8 \mathrm{~nm}$ and power of $0.5 \mathrm{~mW}$ passed through the LN:Fe crystal of $10 \mathrm{~mm}$ thickness when the crystal temperature has been increased from 10 to $45^{\circ} \mathrm{C}$. (f) and (g) are computer treatments of the patterns shown in (a) and (e) with the use of Origin program by scanning the beam traces along horizontal $\mathrm{X}$ and vertical $\mathrm{Y}$ (C-axis) directions. (e) and (g) demonstrate the beam pronounced deflection and self-trapping. The scale shown in Fig. 5(a) is valid for all figures (b)-(e). 
Fig. 6. Experimental demonstration of pyroelectric assisted beam FWHM (circles) narrowing and intensity (triangles) variation with time along transverse $\mathrm{X}(\mathrm{a}, \mathrm{b})$ and $\mathrm{Y}(\mathrm{C}$-axis of the crystal) $(\mathrm{c}, \mathrm{d})$ directions. The larger size circles and triangles show the values at $t=0$. The increase of the beam FWHM in the time interval of $0-5 \mathrm{~s}$ in (c) is connected with the stretching of optical beam along crystal C-axis. The total beam splitting takes place at time instant of 7 s. Subsequent measurements of the beam FWHM were performed for the lower lobe. The intensity decrease at $t=7 \mathrm{~s}$ in (b), (d) is connected with beam splitting along $\mathrm{C}$-axis of the crystal. The solid curves are the fit of experimental dependences by corresponding functions.

Fig. 7. Time dependence of the self-trapped optical beam self-deflection $\Delta Y$ relative to the input beam center, depicted in Figs. 5(a)-(e). Experiment (squares); fit (solid curve). The standard deviation of the measured values is estimated around $10 \%$.

Fig. 8. (a) Schematics of refractive indices $(\delta n)_{\mathrm{PV}}$ and $(\delta n)_{\mathrm{PY}}$ variation by photovoltaic and pyroelectric fields, respectively, along the crystal $\mathrm{C}$-axis taking into account spatial temperature gradient during crystal heating from the bottom side ( $-\mathrm{C}$ pole). (b) For $E_{\mathrm{PY}}>E_{\mathrm{PV}}$ the resulting pyroelectric field induced refractive index change has maximum shifted relative to the beam center toward the $-\mathrm{C}$ side of the crystal because of its relatively higher temperature.

Fig. 9. Simulated $10 \mathrm{~mW}$ power Gaussian beam intensity distribution with waist of $72 \mu \mathrm{m}$ (a) at the input of $\mathrm{LN}$ :Fe sample $(t=0)$. The simulation results of refractive index change $\Delta n(\mathrm{~b})$ and evolution of laser beam intensity in $\mathrm{Y}-\mathrm{Z}$ plane (c) along beam propagation through the $10 \mathrm{~mm}$ thick crystal at $t=35 \mathrm{~s}$. The intensity profile in the transverse $\mathrm{X}-\mathrm{Y}$ plane (d) and its distribution along $\mathrm{Y}$ 
(C-axis) direction (e) for instant of $t=35 \mathrm{~s}$. In (e) the central contour is the output intensity profile along $\mathrm{Y}$ at $t=0$ (the intensities are given in arbitrary units). Simulations are performed for the epolarized input laser beam. In (d) the scales in units of meter along $\mathrm{X}$ and $\mathrm{Y}$ are different.

Fig. 10. Simulation results of refractive index change $\Delta n$ (a) and evolution of laser beam intensity in Y-Z plane (b) along beam propagation through the $10 \mathrm{~mm}$ thick crystal at $t=80 \mathrm{~s}$. The intensity profile in the transverse $\mathrm{X}-\mathrm{Y}$ plane (c) and its distribution along $\mathrm{Y}(\mathrm{C}$-axis) direction (d) for instant of $t=80 \mathrm{~s}$. In (d) the central contour is the output intensity profile along Y direction at $t=0$ (the intensities are given in arbitrary units). Simulations are performed for the e-polarized input laser beam.

Fig. 11. Simulated time dependence of self-localized beam deflection $\Delta \mathrm{Y}_{\mathrm{sml}}$ relative to the output beam centre at $t=0$ (see Fig. 11(d)). Solid line shows the linear increase of beam deflection in the simulated time range of 0-60 s and the dashed lines depicts the deviation of deflection from linear dependence for 60-120 s with tendency of saturation for further time evolution. 

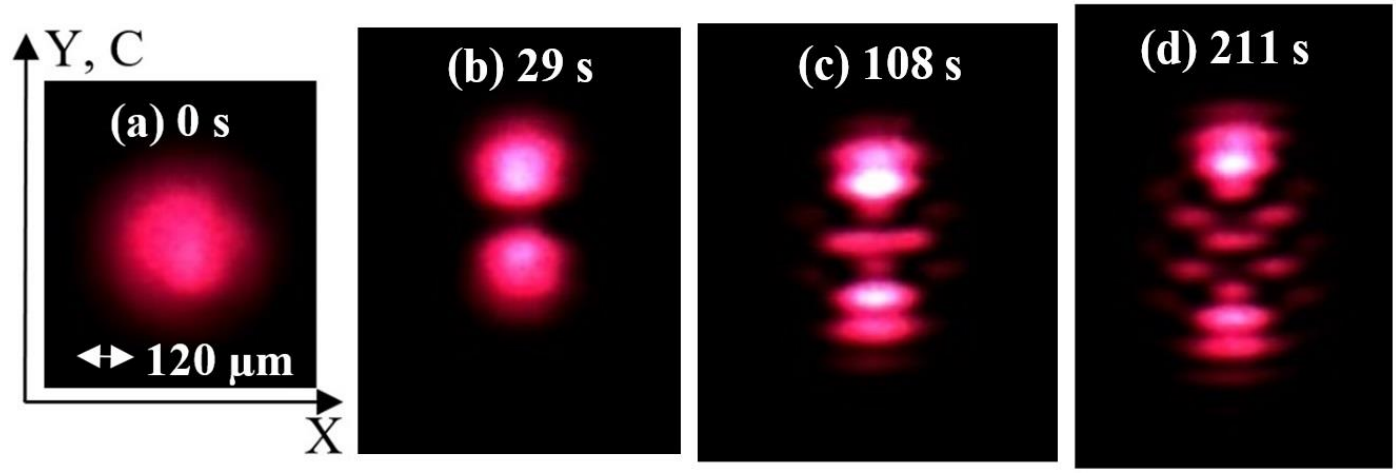

Lusine Tsarukyan, Anahit Badalyan, Fabrice Devaux, Mathieu Chauvet and Rafael Drampyan, Pyroelectric-controlled bending of a self-trapped optical beam in a photorefractive iron doped lithium niobate crystal, Fig. 1. 


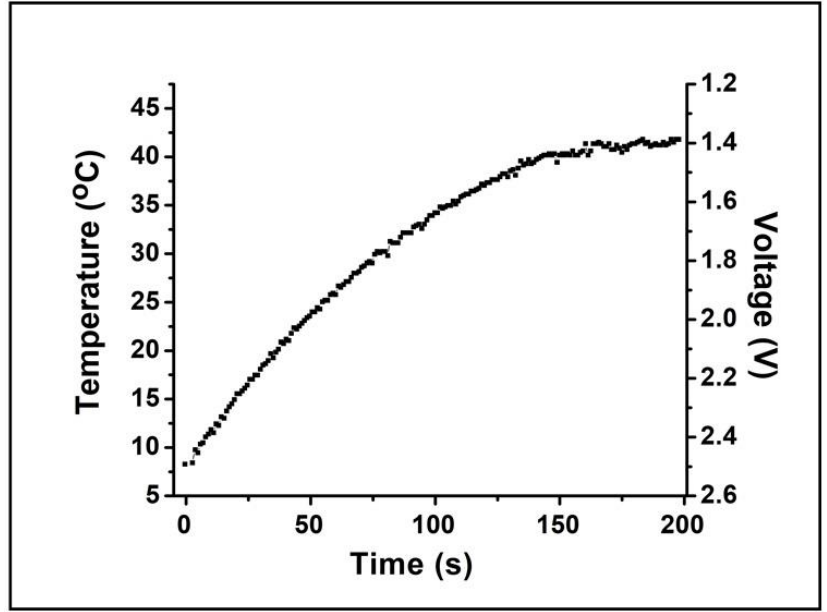

Lusine Tsarukyan, Anahit Badalyan, Fabrice Devaux, Mathieu Chauvet and Rafael Drampyan, Pyroelectric-controlled bending of a self-trapped optical beam in a photorefractive iron doped lithium niobate crystal, Fig. 2. 


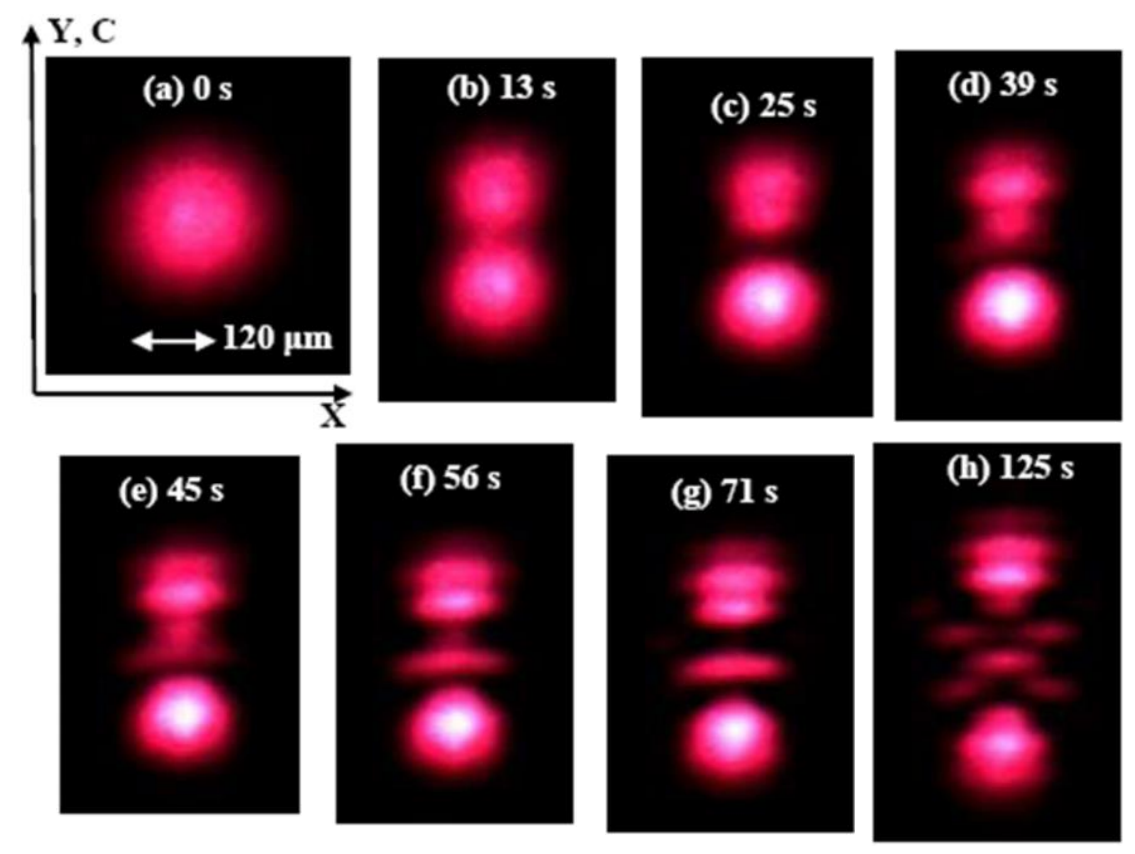

Lusine Tsarukyan, Anahit Badalyan, Fabrice Devaux, Mathieu Chauvet and Rafael Drampyan, Pyroelectric-controlled bending of a self-trapped optical beam in a photorefractive iron doped lithium niobate crystal, Fig. 3. 


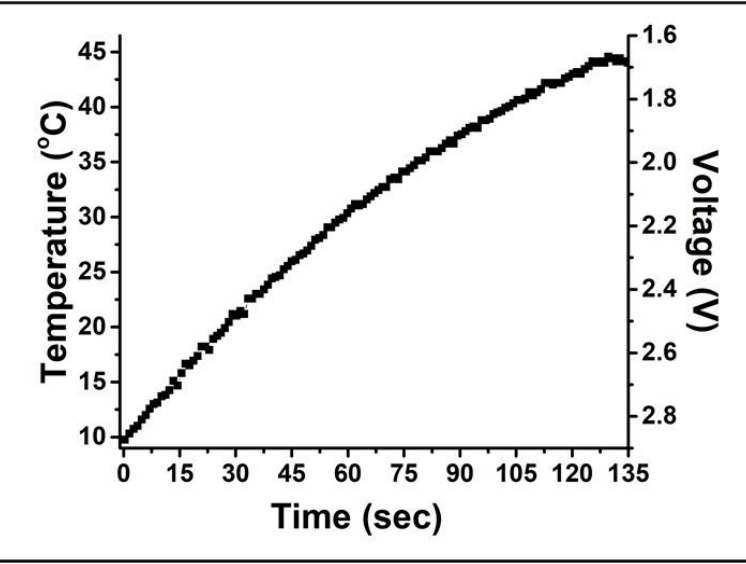

Lusine Tsarukyan, Anahit Badalyan, Fabrice Devaux, Mathieu Chauvet and Rafael Drampyan, Pyroelectric-controlled bending of a self-trapped optical beam in a photorefractive iron doped lithium niobate crystal, Fig. 4. 


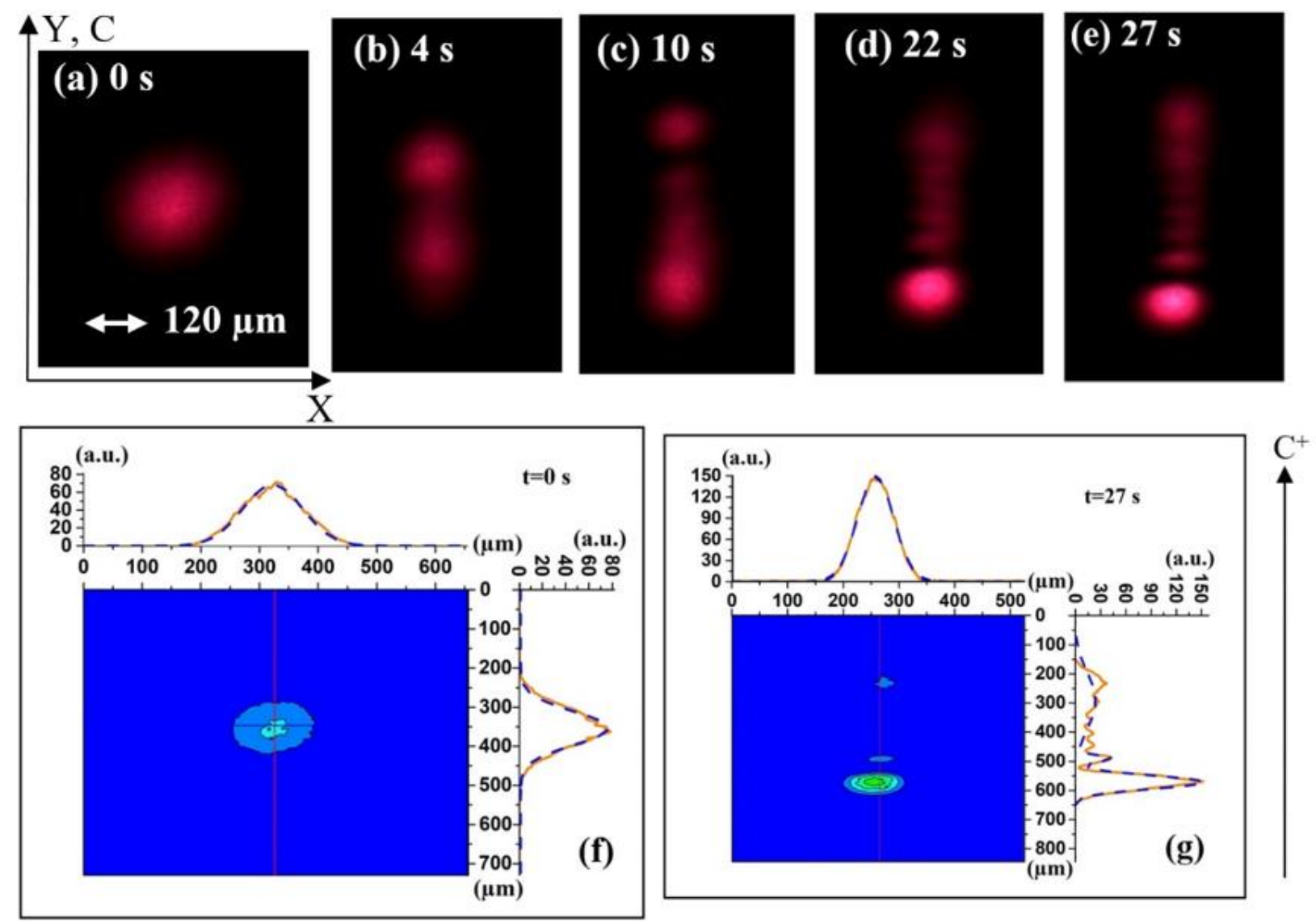

Lusine Tsarukyan, Anahit Badalyan, Fabrice Devaux, Mathieu Chauvet and Rafael

Drampyan, Pyroelectric-controlled bending of a self-trapped optical beam in a photorefractive iron doped lithium niobate crystal, Fig. 5. 

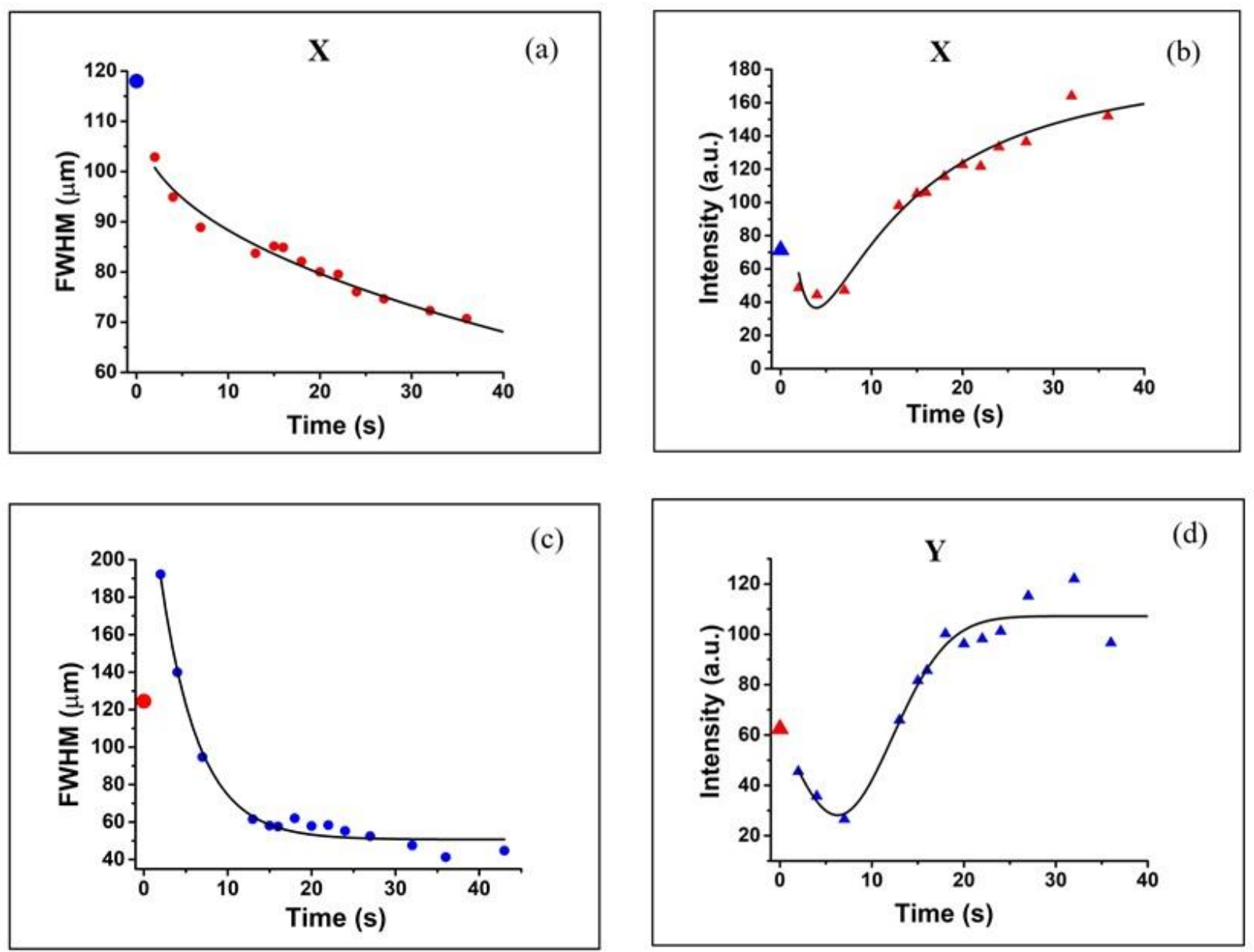

Lusine Tsarukyan, Anahit Badalyan, Fabrice Devaux, Mathieu Chauvet and Rafael Drampyan, Pyroelectric-controlled bending of a self-trapped optical beam in a photorefractive iron doped lithium niobate crystal, Fig. 6. 


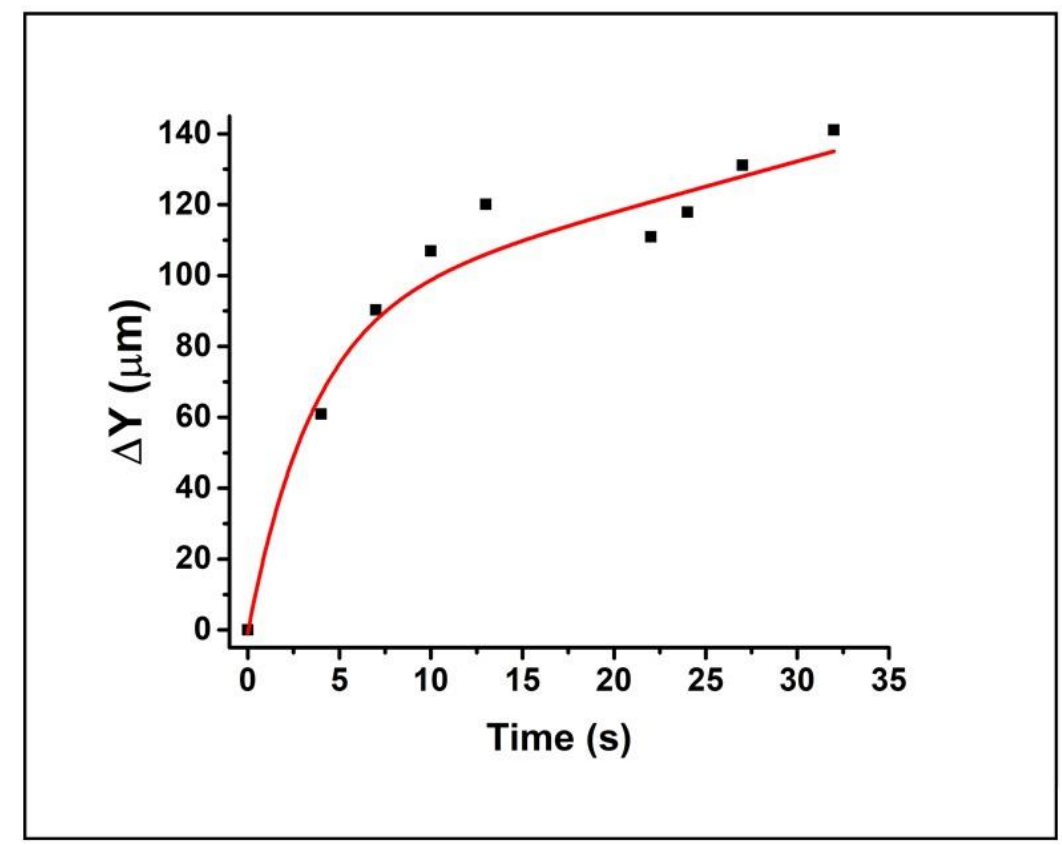

Lusine Tsarukyan, Anahit Badalyan, Fabrice Devaux, Mathieu Chauvet and Rafael Drampyan, Pyroelectric-controlled bending of a self-trapped optical beam in a photorefractive iron doped lithium niobate crystal, Fig. 7. 


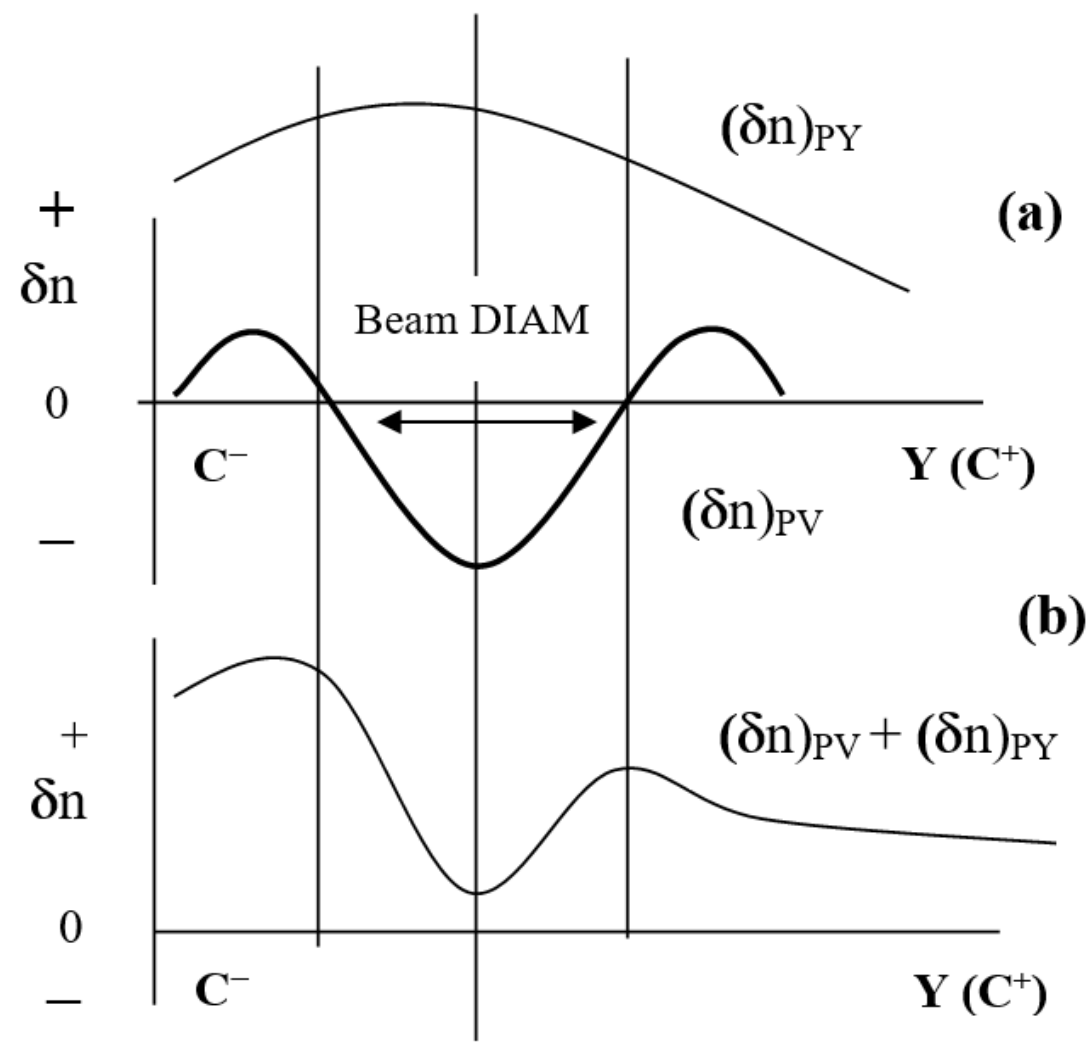

Lusine Tsarukyan, Anahit Badalyan, Fabrice Devaux, Mathieu Chauvet and Rafael Drampyan, Pyroelectric-controlled bending of a self-trapped optical beam in a photorefractive iron doped lithium niobate crystal, Fig. 8. 


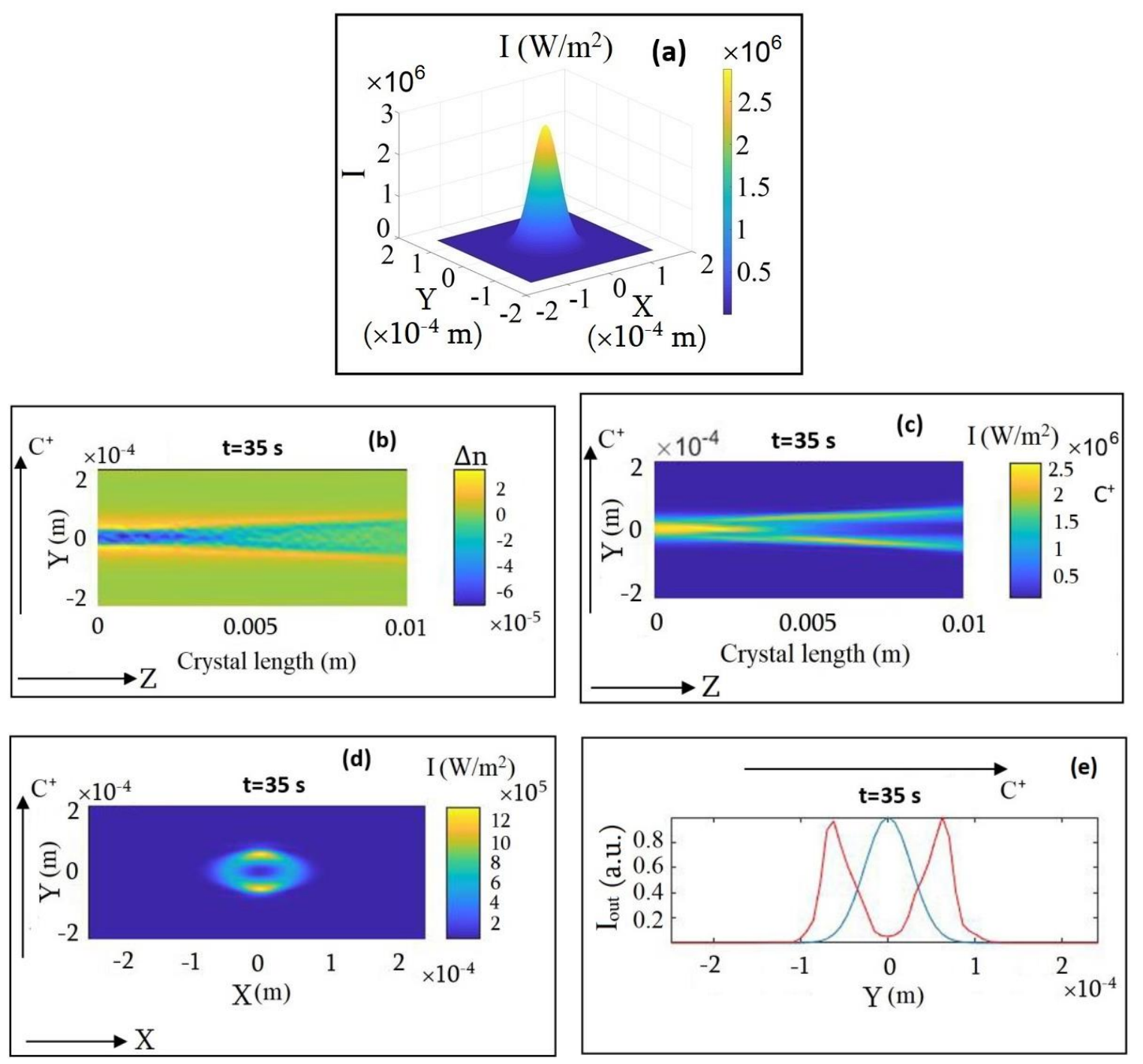

Lusine Tsarukyan, Anahit Badalyan, Fabrice Devaux, Mathieu Chauvet and Rafael Drampyan, Pyroelectric-controlled bending of a self-trapped optical beam in a photorefractive iron doped lithium niobate crystal, Fig. 9. 

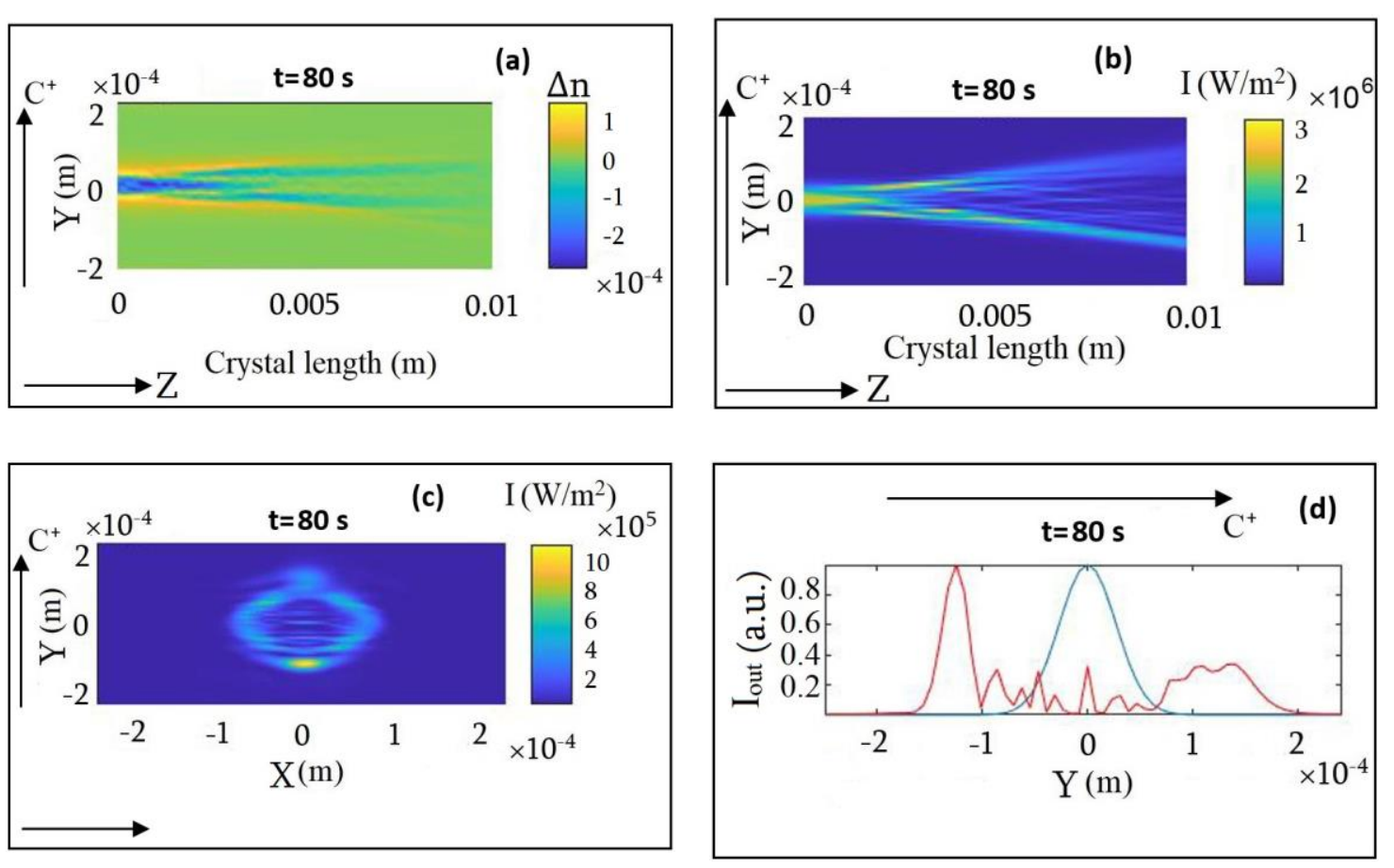

Lusine Tsarukyan, Anahit Badalyan, Fabrice Devaux, Mathieu Chauvet and Rafael Drampyan, Pyroelectric-controlled bending of a self-trapped optical beam in a photorefractive iron doped lithium niobate crystal, Fig. 10. 


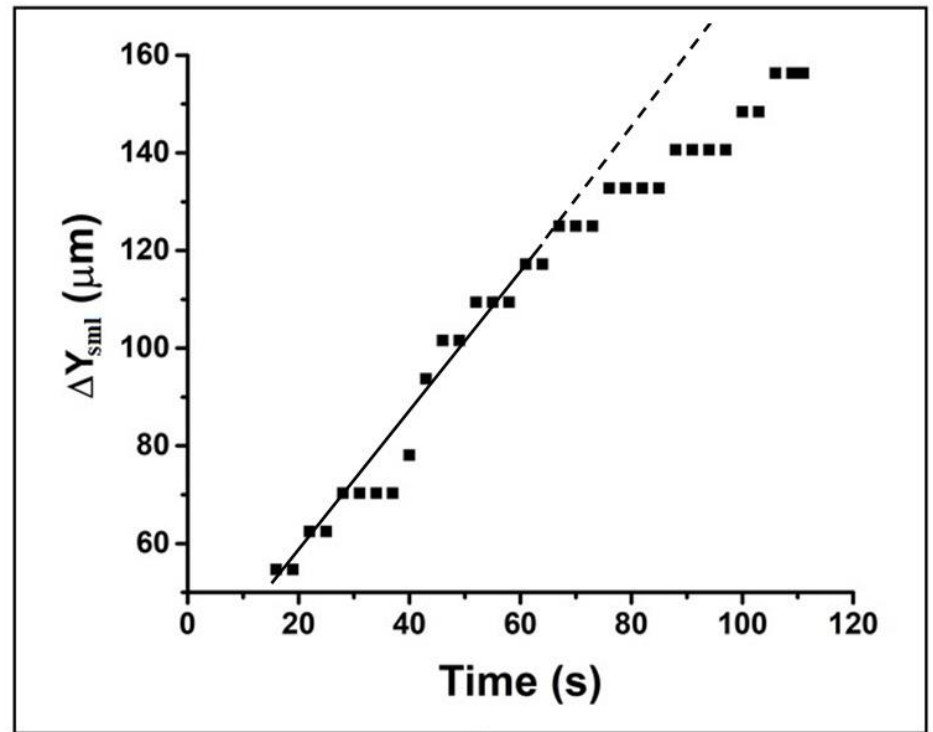

Lusine Tsarukyan, Anahit Badalyan, Fabrice Devaux, Mathieu Chauvet and Rafael Drampyan, Pyroelectric-controlled bending of a self-trapped optical beam in a photorefractive iron doped lithium niobate crystal, Fig. 11. 


\section{Answers to the reviewers' comments concerning the manuscript}

Pyroelectric-controlled bending of a self-trapped optical beam in a photorefractive iron doped lithium niobate crystal

by Lusine Tsarukyan, Anahit Badalyan, Fabrice Devaux, Mathieu Chauvet, Rafael Drampyan

The authors are thankful to the reviewers for careful reading of the Manuscript and constructive comments and suggestions.

\section{Reviewer \#1:}

I read with great attention the manuscript JNOPM-D-21-00032 "Pyroelectric-controlled bending of a self-trapped optical beam in a photorefractive iron doped lithium niobate crystal" by L. Tsarukyan and al. The manuscript present both a theoretical and experimental investigation on the possibility to control the natural bending of pyrolitons (pyroelectric solitons) in lithium niobate doped with iron.

The paper is well structured, clear and solve and important issue for both physical knowledge and technological applications of self-trapped beam.

Theory well describe the experimental results. I would recommend the publication of the paper as is.

\section{Answer:}

The authors are grateful to the reviewer for high estimation of the paper and recommendation for publication of the paper as is.

\section{Reviewer \#2}

The authors have performed a comprehensive investigation of self-trapped optical beam bending in a photorefractive Fe doped lithium niobate crystal controlled by the pyroelectric effect. The authors explain the experimental observations like the bending being opposite to the crystal $\mathrm{C}+$ axis satisfactorily. The numerical simulations agree with the experimental observation. The work is technically correct and novel to warrant publication in JNOPM. I would, however like to suggest a few minor changes as follows:

1. In the Section 1.Introduction, there are several recent investigations which are relevant to the pyroelectric effect in lithium niobate and pyroelectric solitons which can be included. I am writing down a few:

-Popescu, S. T., A. Petris, and V. I. Vlad. "Experimental investigation of the output mode profile of soliton waveguides recorded at $405 \mathrm{~nm}$ wavelength in lithium niobate." Rom. Rep. Phys 63 (2013): 915-924.

-Katti, Aavishkar, and R. A. Yadav. "Spatial solitons in biased photovoltaic photorefractive materials with the pyroelectric effect." Physics Letters A 381.3 (2017): 166-170. -Jiang, Qichang, Yanli Su, and Xuanmang Ji. "Pyroelectric photovoltaic spatial solitons in unbiased photorefractive crystals." Physics Letters A 376.45 (2012): 3085-3087. -Katti, Aavishkar. "Gaussian soliton pairs in an unbiased photorefractive crystal due to the pyroelectric effect." The European Physical Journal Plus 134.12 (2019): 1-11. -Popescu, S. T., A. Petris, and V. I. Vlad. "Fast writing of soliton waveguides in lithium niobate using low-intensity blue light." Applied Physics B 108.4 (2012): 799-805.

Answer: We apologize for missing of a several recent publications which are relevant to the pyroelectric effect in lithium niobate and pyroelectric solitons.

We included the first recommended paper in the Introduction, page 2, as Ref.11. 
We added the next three recommended papers in the Introduction of MS (page 3, lines 1-2 from the top) with the following sentence:

Pyroelectric photovoltaic spatial solitons in unbiased photorefractive crystals have been studied further theoretically in Ref.18 -20.

The recommended Ref. -Popescu, S. T., A. Petris, and V. I. Vlad. "Fast writing of soliton waveguides in lithium niobate using low-intensity blue light." Applied Physics B 108 (2012): 799-805 is cited in the Conclusion of MS (page 15, lines 7-8) with the following description:

The long dark-storage time soliton waveguide in undoped LN crystal was reported before in Ref. 44.

All corrections in the numeration of Ref. numbers are highlighted by red font.

2. In Section 5. Numerical Simulations, the authors refer to the theory model of Ref.37 for explaining the source of their numerical simulations. It would be better if the authors include the theoretical modelling equations explicitly for the present case showing the contribution of the pyroelectric effect.

As requested by the reviewer, we have inserted the system of equations (2) that is numerically solved. The different parameters are also described along with a few sentences to clarify the contribution of the pyroelectric field, which is one component of the total electric field.

The following new text is included in the MS from line 7 from the top on page 11 to line 4 from the top on page 12 which is highlighted by red font:

The following two-photoactive- center model is applied for simulations of selffocusing in $\mathrm{LN}: \mathrm{Fe}$ crystal under pyroelectric effect:

$$
\begin{gathered}
\frac{\partial N_{F e}^{+}}{\partial t}=\left(s_{F e}+s_{F e X} N_{X}^{+}\right) I\left(N_{F e}-N_{F e}^{+}\right)-\left[\gamma_{F e} N_{e}+\gamma_{X F e}\left(N_{X}-N_{X}^{+}\right)\right] N_{F e}^{+} \\
\frac{\partial N_{X}^{+}}{\partial t}=\left(s_{X} I+\gamma_{X F e} N_{F e}^{+}\right)\left(N_{X}-N_{X}^{+}\right)-\left[\gamma_{X} N_{e}+s_{F e X} I\left(N_{F e}-N_{F e}^{+}\right)\right] N_{X}^{+} \\
\frac{\partial N_{e}}{\partial t}=\frac{\partial N_{F e}^{+}}{\partial t}+\frac{\partial N_{X}^{+}}{\partial t}-\frac{1}{e} \frac{\partial \rho}{\partial t} \\
\vec{J}=e \mu N_{e} \vec{E}+k_{F e} I\left(N_{F e}-N_{F e}^{+}\right) \vec{c}+k_{X} I\left(N_{X}-N_{X}^{+}\right) \vec{c}+\mu k_{B} T \vec{\nabla} N_{e} \\
\frac{\partial \rho}{\partial t}=-\vec{\nabla} \cdot \vec{J}
\end{gathered}
$$

where $\mathrm{Ne}, \mathrm{N}_{\mathrm{Fe}}$, and $\mathrm{N}^{+} \mathrm{X}$ are, respectively, the densities of free electrons, ionized iron, and ionized polarons. $I$ is the light intensity, $s_{\mathrm{Fe}}, s_{X}$, and $s_{\mathrm{Fe} X}$ are the photoionization cross sections of electrons from, respectively, the $\mathrm{Fe}^{2+}$ and $X$ states into the conduction band and from $\mathrm{Fe}^{2+}$ to $X^{+}$states. $\gamma_{\mathrm{Fe}}, \gamma_{X}$, and $\gamma_{X \mathrm{Fe}}$ are the corresponding recombination rates of electrons. Equation (2d) is the expression of the total current density $J$ where drift current, photovoltaic (PV) currents of both photoactive centers, and thermal diffusion are considered. $\kappa_{\mathrm{Fe}}$ and $\kappa_{X}$ are the PV coefficients. $\vec{E}$ represents the total electric field in the material, including the pyroelectric field component $E_{\mathrm{py}}$ which is oriented along the crystal c-axis. $E_{\mathrm{py}}$ is calculated using equation (1). The space-charge density is $\rho=e\left(N^{+} \mathrm{Fe}+N^{+}{ }_{X}-N_{A}-N_{e}\right)$, where the constant acceptor 
concentration $N_{A}$ corresponds to the shallow acceptor density. $e$ and $\mu$ are, respectively, the elementary charge and the mobility of electrons. $k_{B}$ is the Boltzmann constant, and $T$ is the temperature.

This system of equations (2) ......

3. Please give a relevant reference for equation (2).

\section{Answer:}

In revised version of the manuscript formula (2) became formula (3). The formula (3) is written intuitively to describe the experimentally realized temperature increase of the crystal by Peltier element.

The formula (3) is one of the possible approximations of the experimental curves shown in Figs. 2 and 4. We deduced this formula from the following reasons. The terms in the parentheses provides the increase of the temperature $T$ for $\mathrm{t}<<\tau$, and further tendency to slow temperature decrease for $t / \tau>>1$ (second term in the brackets).

We made the following minor revision in the text on page 12 (lines 6 and 4 from the bottom) (highlighted by red font).

"The heating temperature rise versus time $t$ we approximated by the formula:

$$
T(t)=T_{0}+\left(T_{p}-T_{0}\right) \cdot\left(\frac{t / \tau}{1+(t / \tau)}+2 \cdot \frac{t / \tau}{[1+(t / \tau)]^{2}}\right)
$$

where $\tau$ is the time to reach the peak temperature. The deduced $T(t)$ dependence provides...."

During corrections in the MS following the remarks of reviewers, we noticed some minor grammatical and stylistic inaccuracies. We corrected them and highlighted by red font:

(See page 9 line 11 from the bottom - "larger"; page 13, line 7 from the bottom - "ionized iron"; page 14, line 9 from the bottom - "between" instead of "at", "demonstrate" instead of "demonstrates"; page 16, line 7 from the top - "pyroelectric"; page 17, Ref. 7 - "Eds" instead of" eds"; page 18, Ref. 17 - "pyroelectric" instead of "pyroelectrc"; page 21 caption to fig. 5 "(f) and (g) are" instead of "(f, g)", "treatments" instead of "treatment", "(e) and (g)" instead of “(e, g)").

We are thankful again the reviewers for careful reading of MS and valuable remarks and hope that corrections made will meet their requirements. 

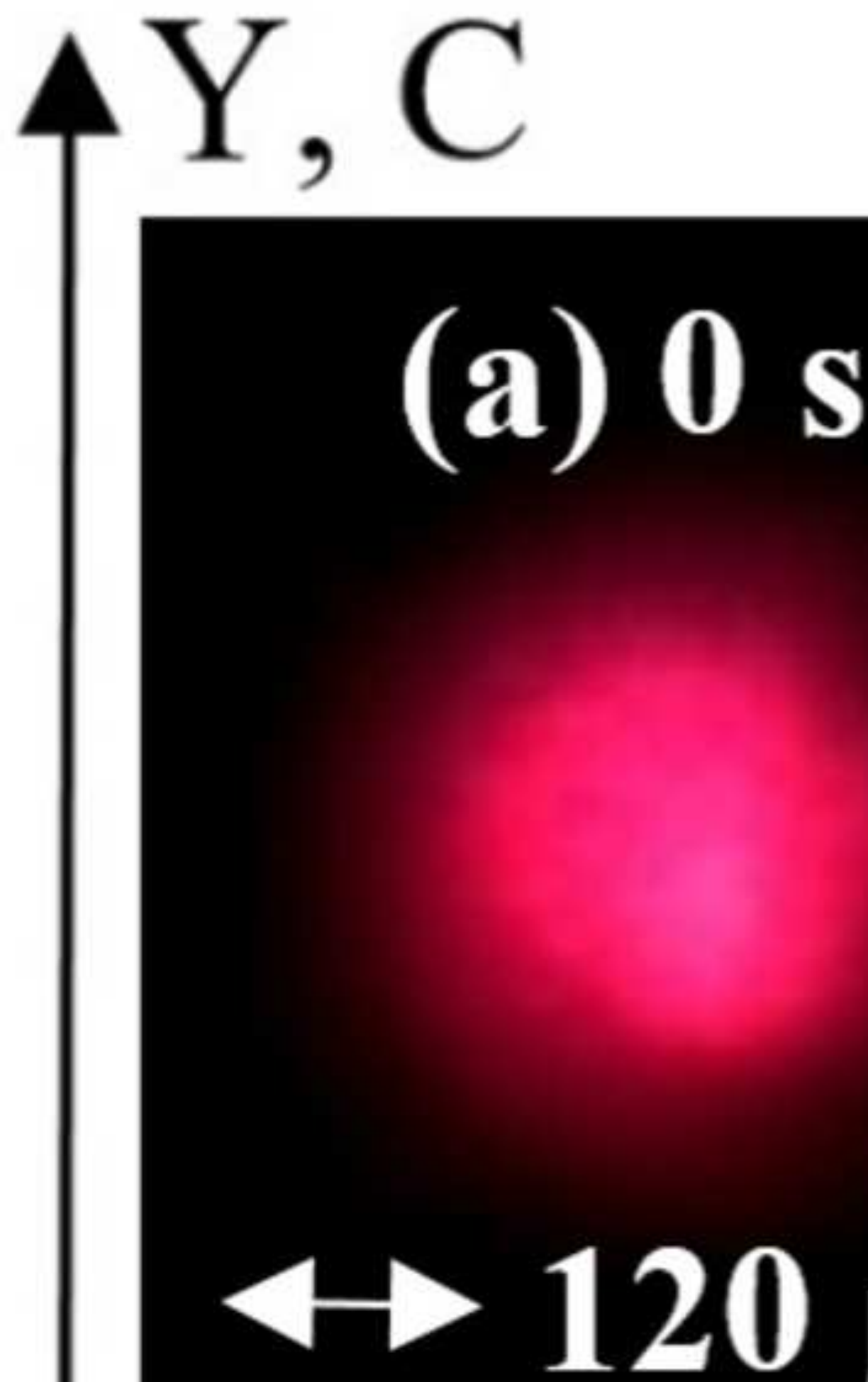

Mm

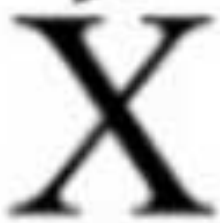




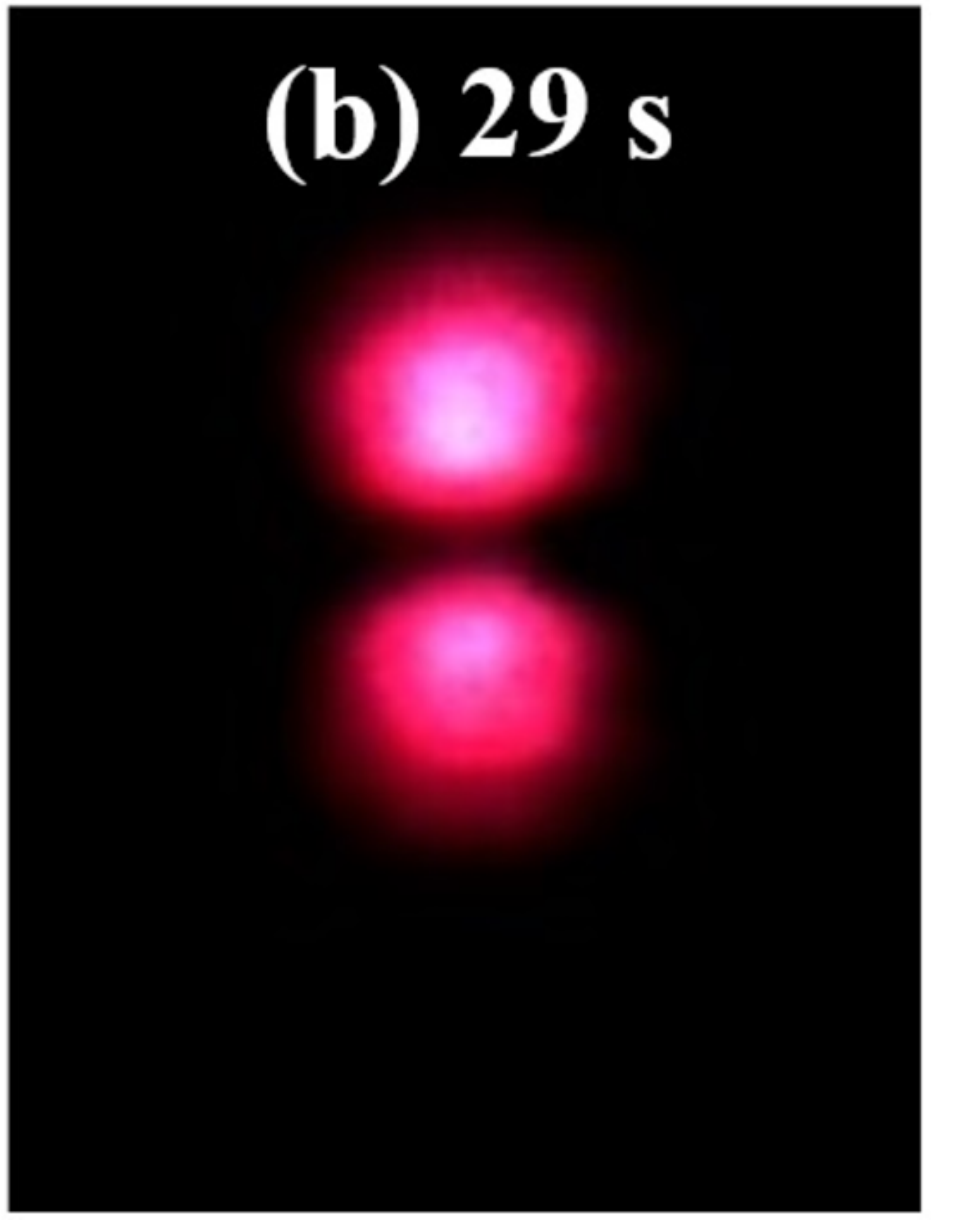
.<smiles>C1CCC1</smiles>
. . . . 


\section{(c) $108 \mathrm{~s}$}

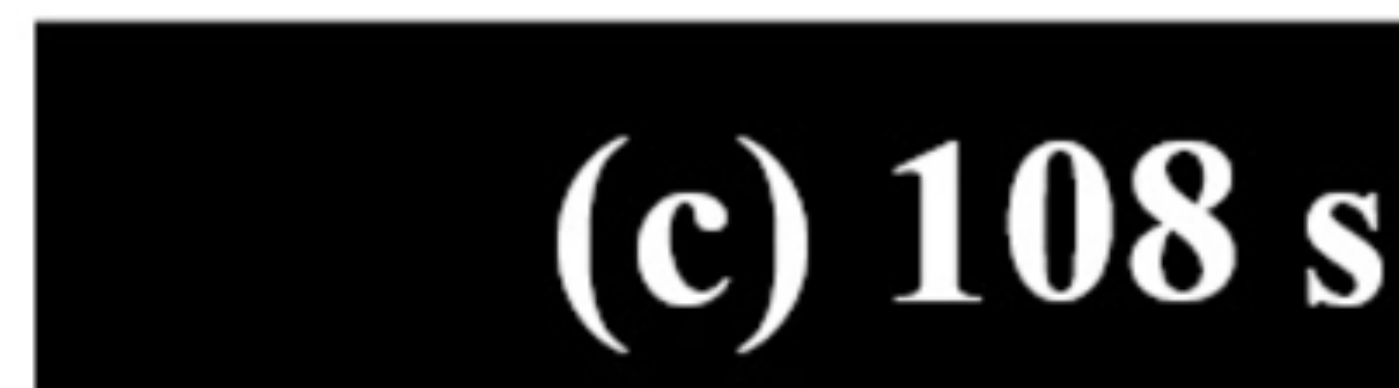




\section{(d) $211 \mathrm{~s}$}

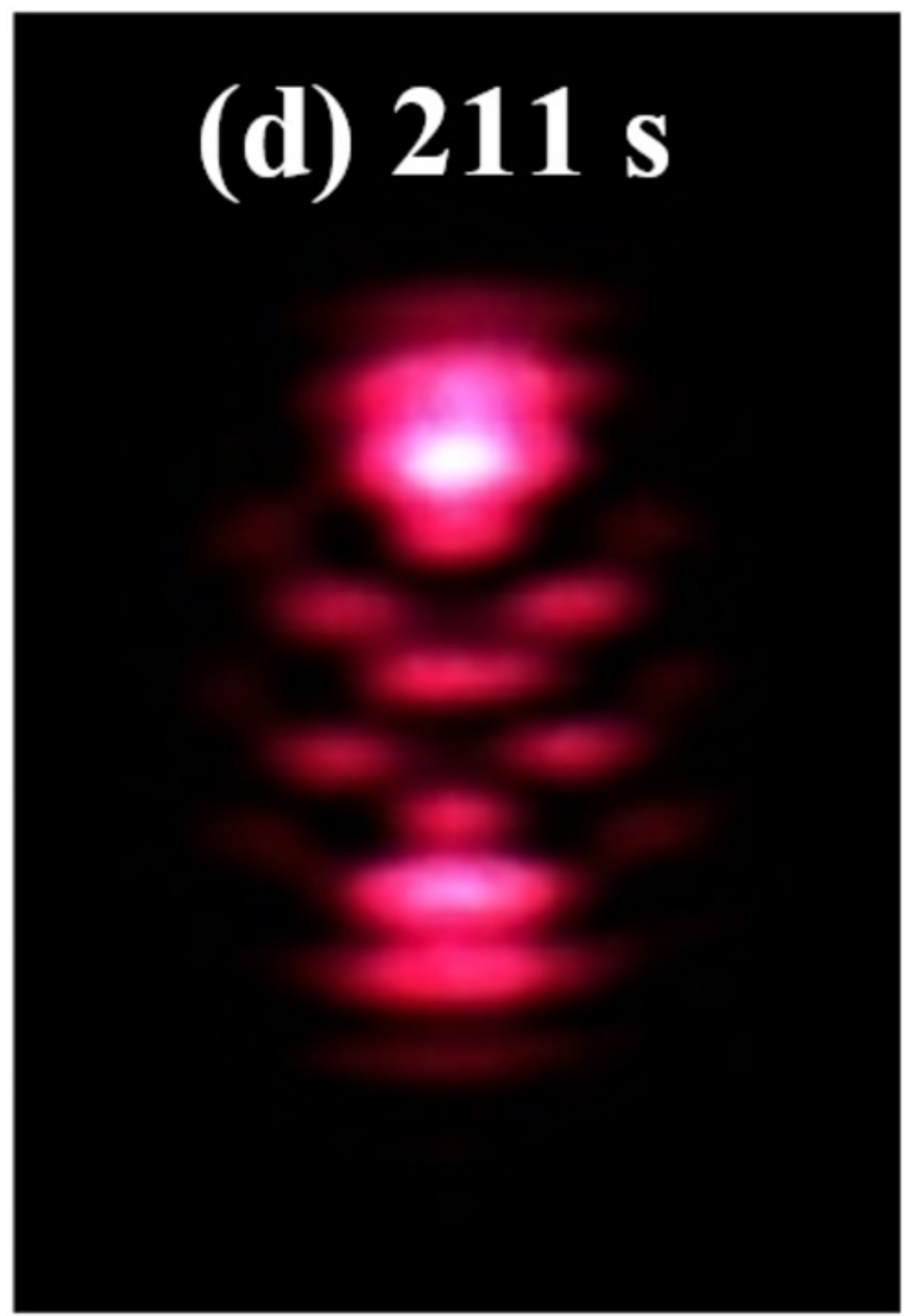




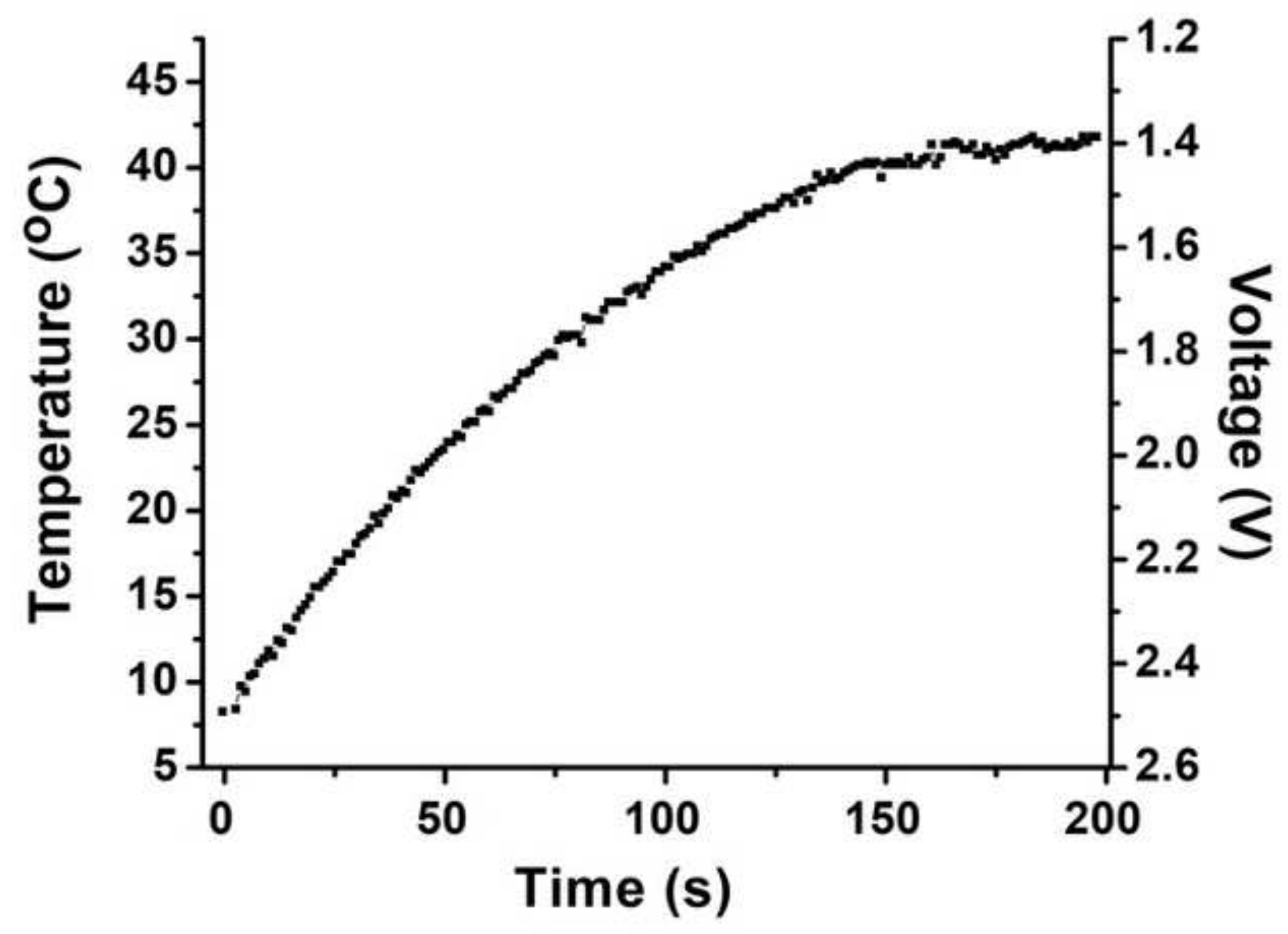




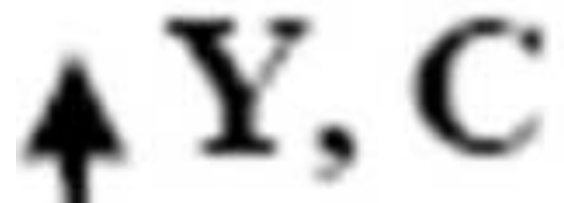

\section{(a) $0 \mathrm{~s}$}
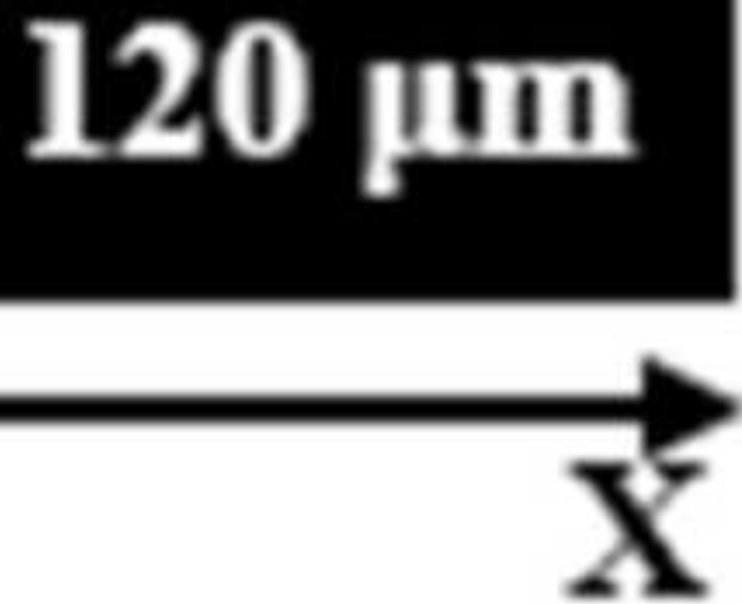


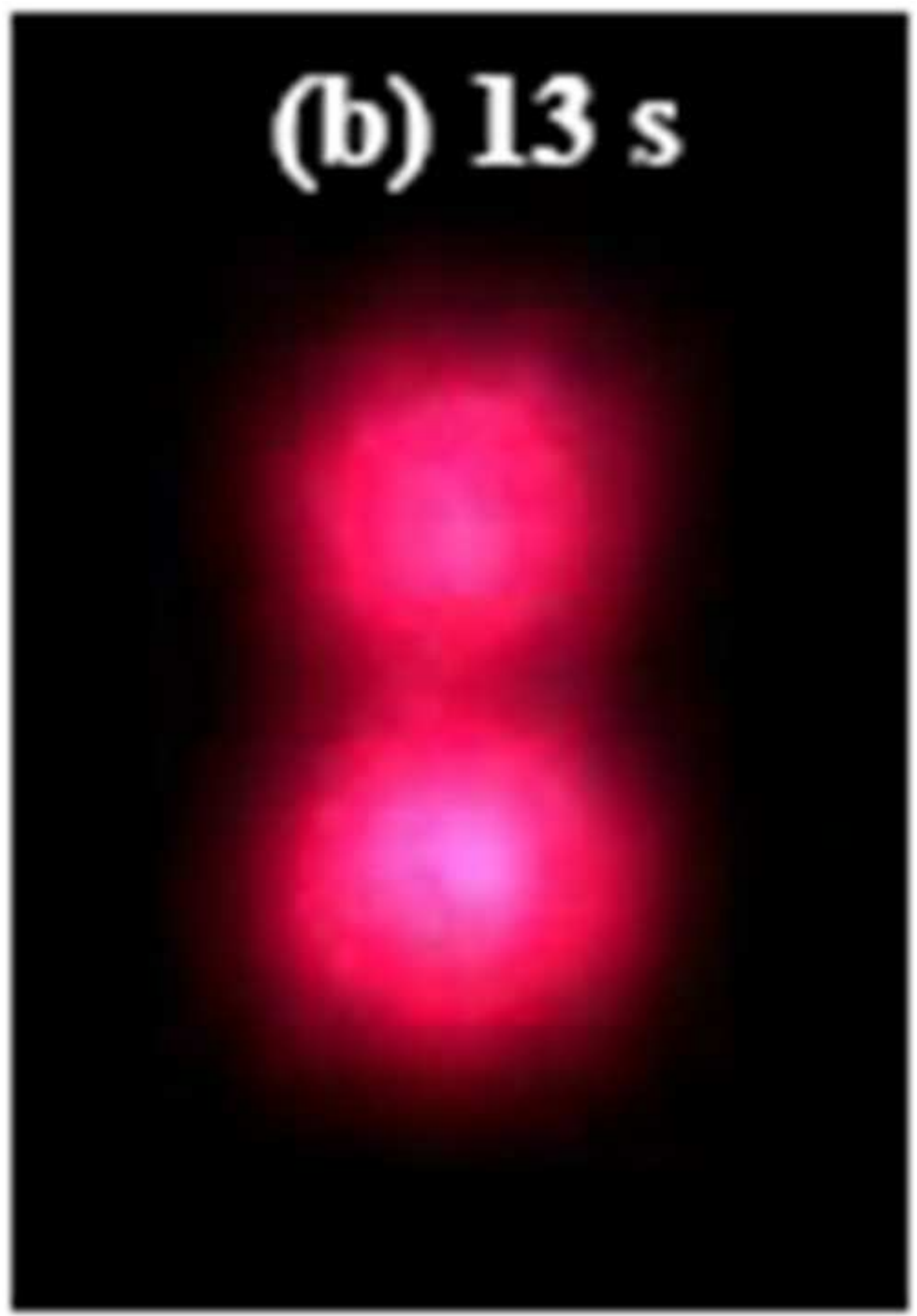




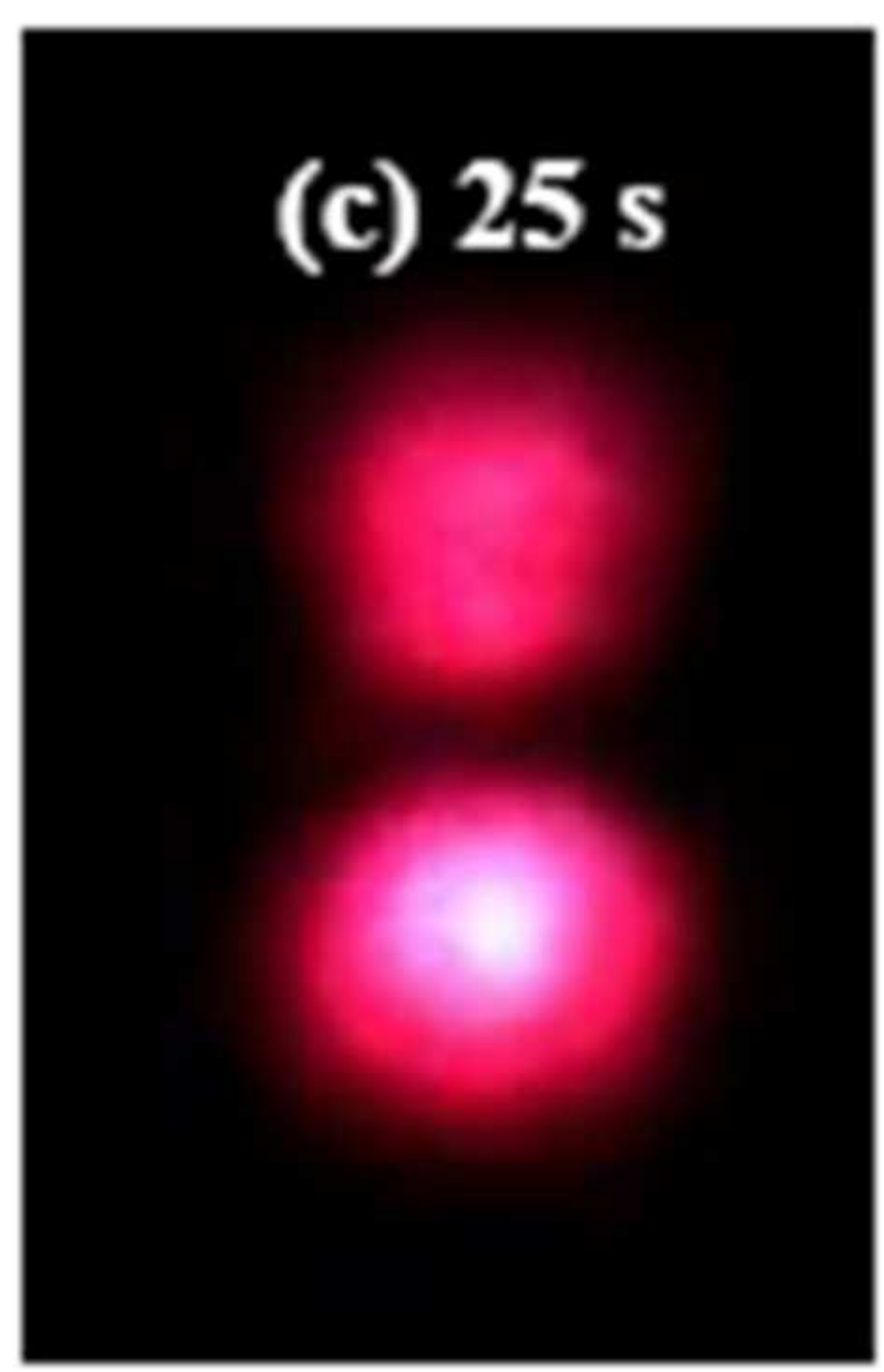




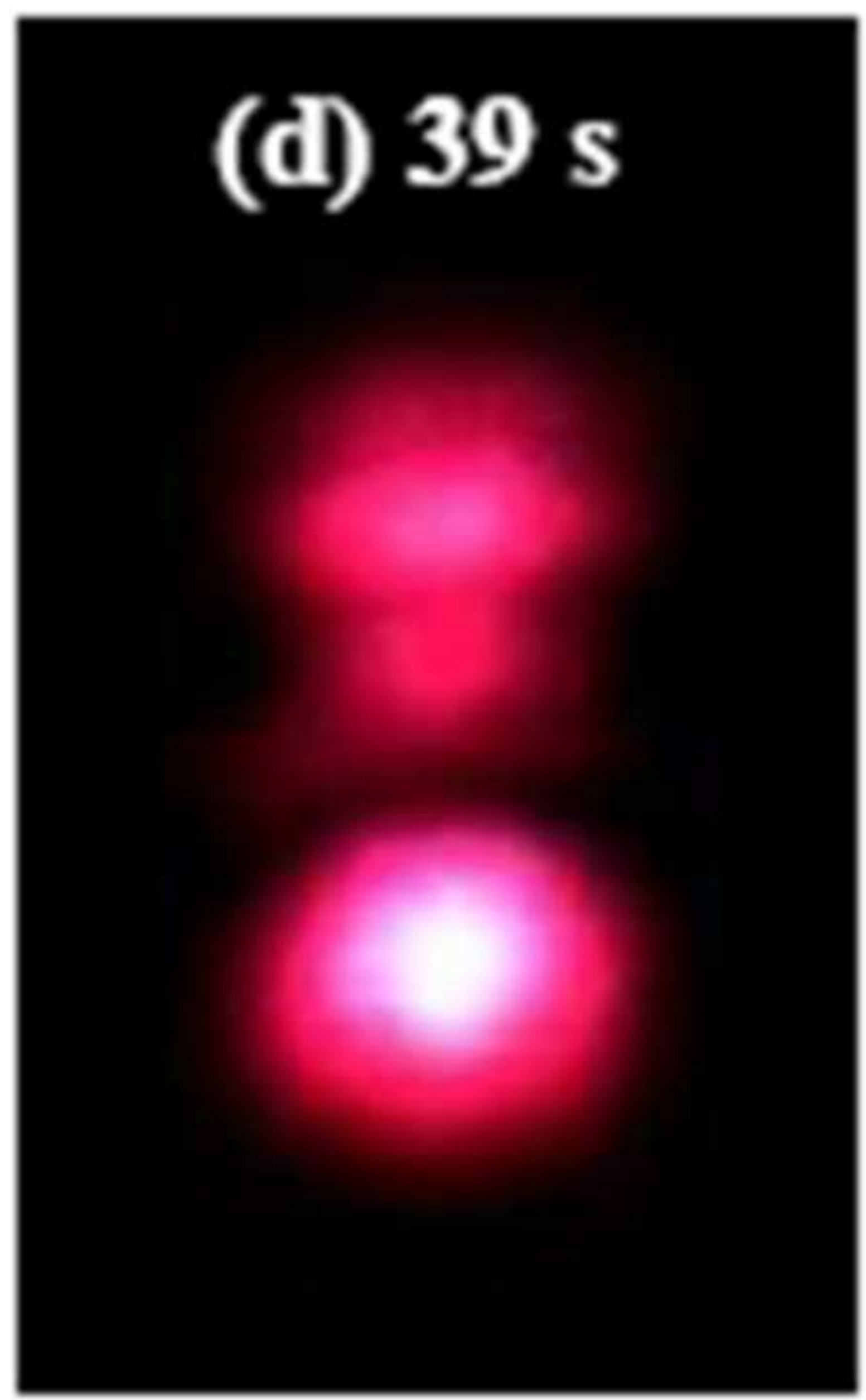




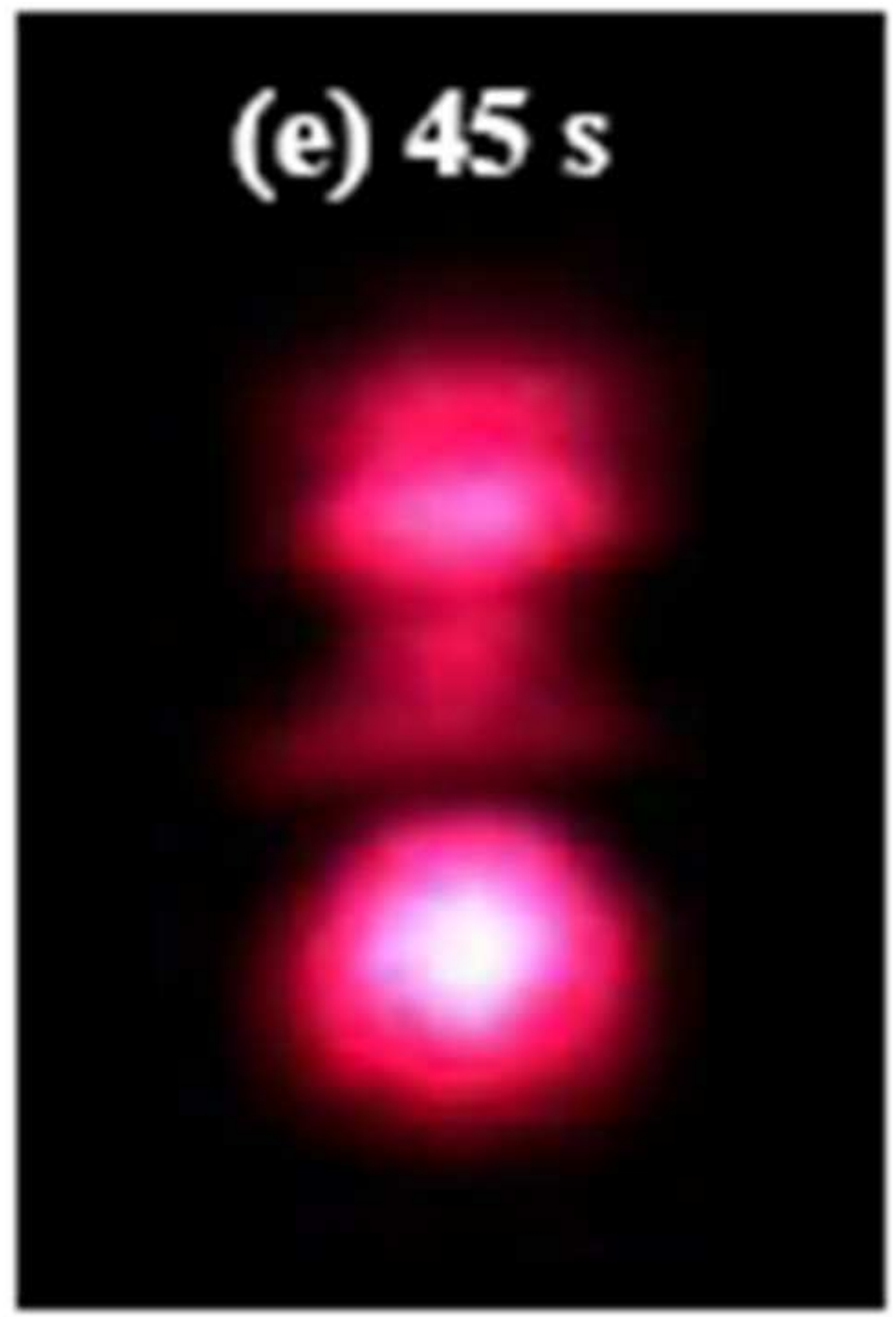




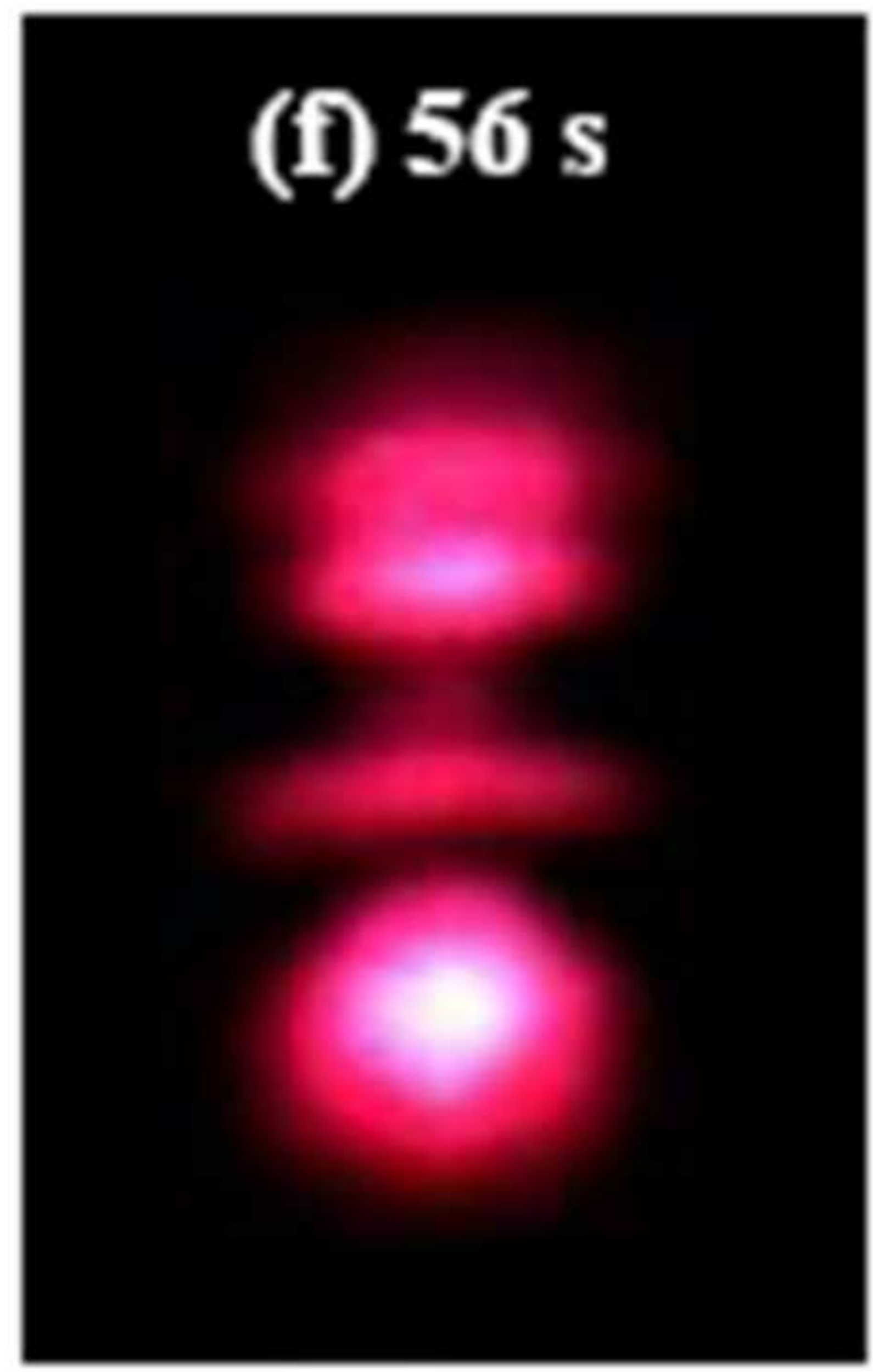




\section{(g) $71 \mathrm{~s}$

Fure 39

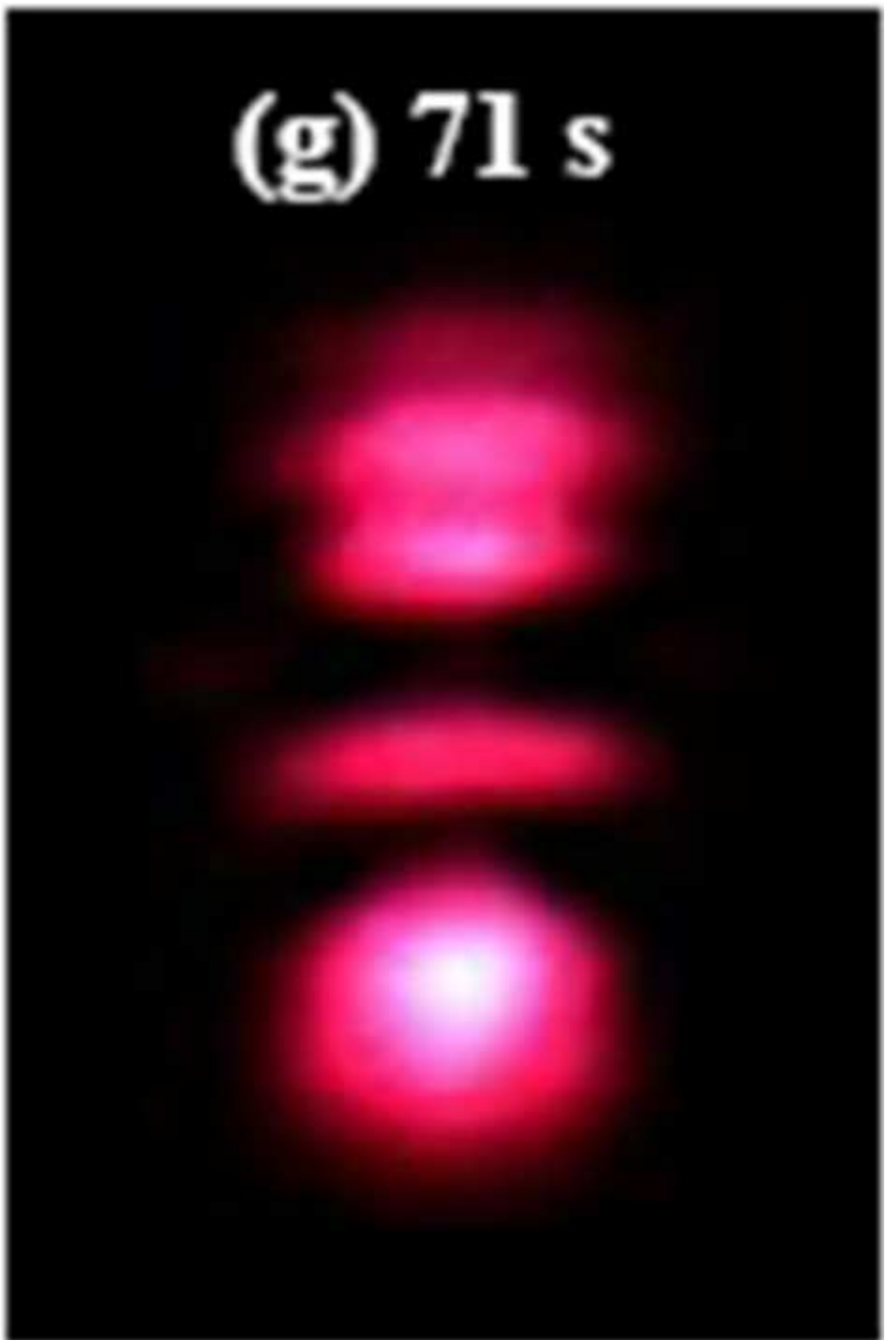




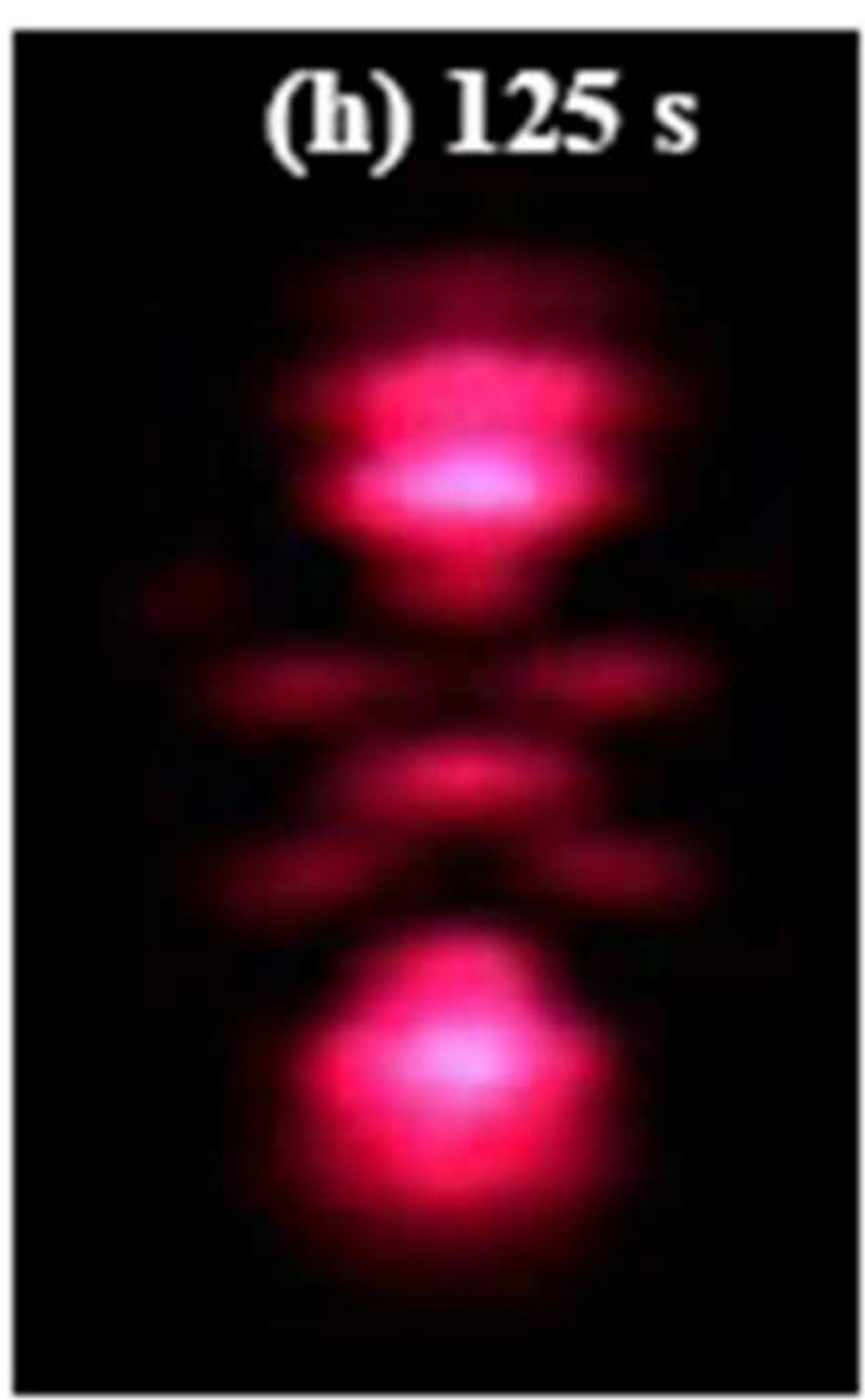




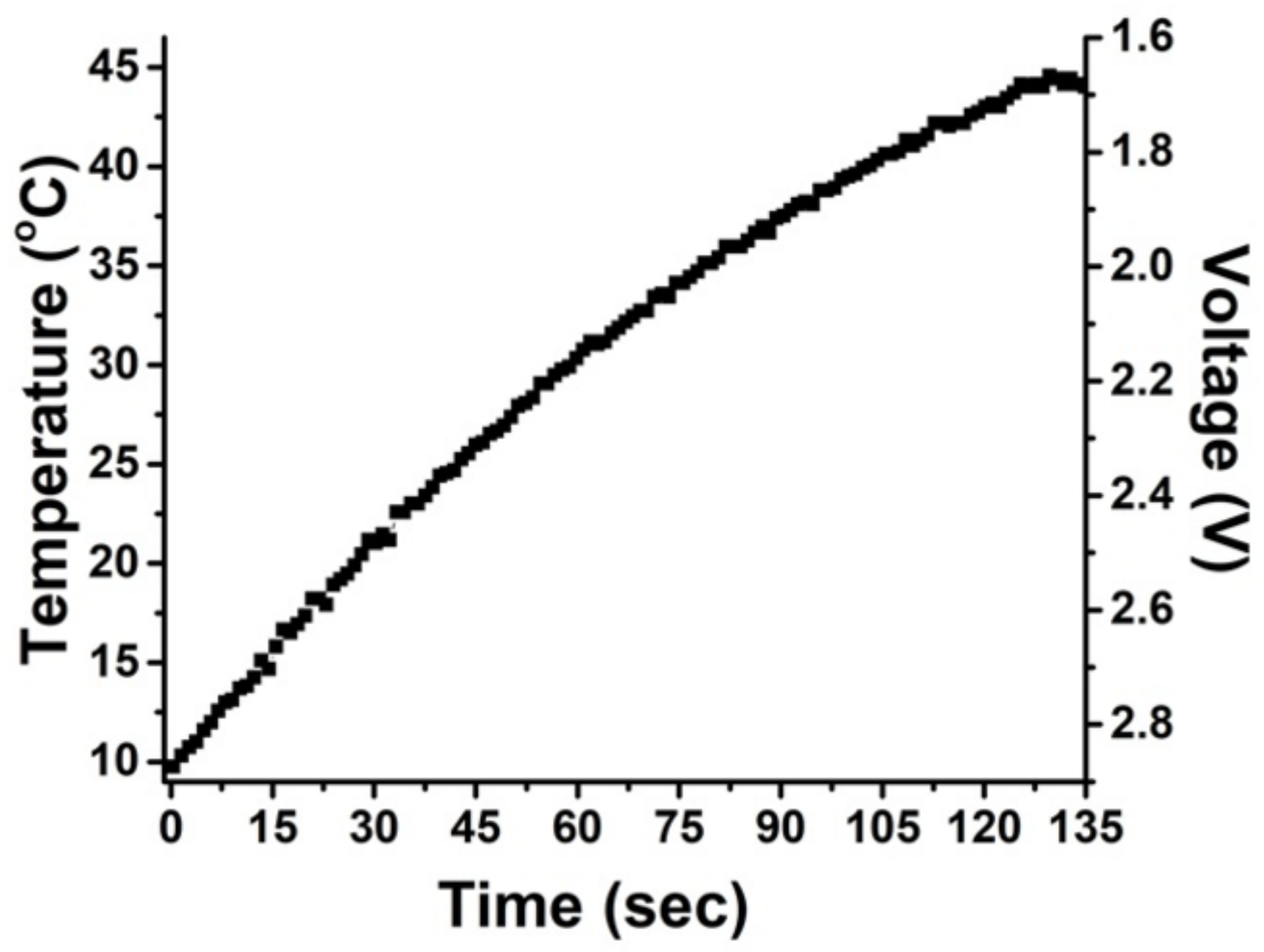




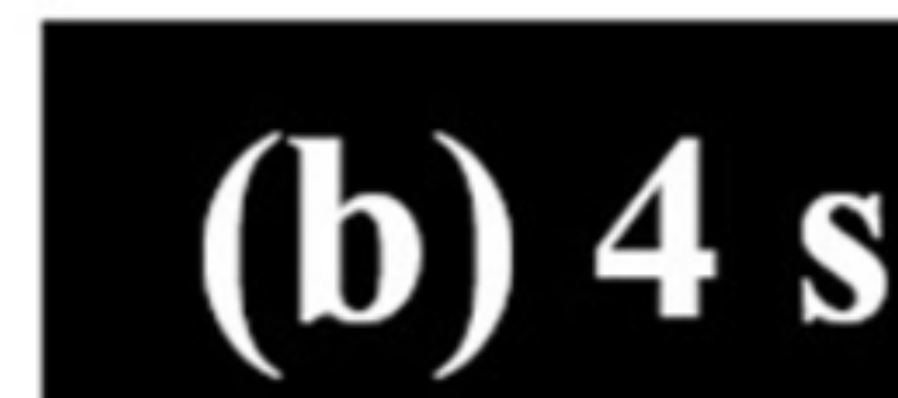

(b) $4 \mathrm{~s}$$$
\text { - }
$$$$
\text { ( }
$$

$$
\mathrm{s}
$$

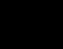




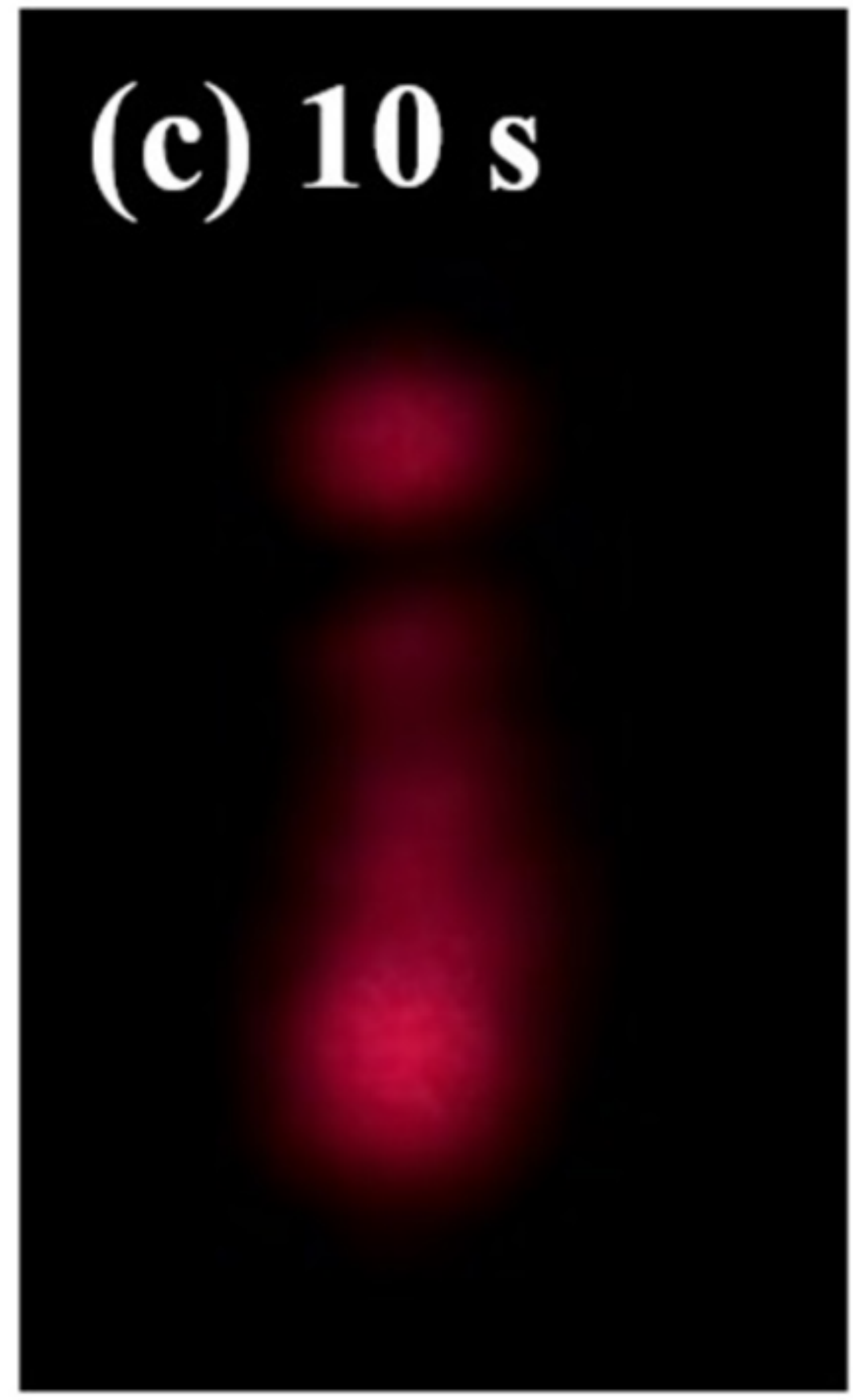




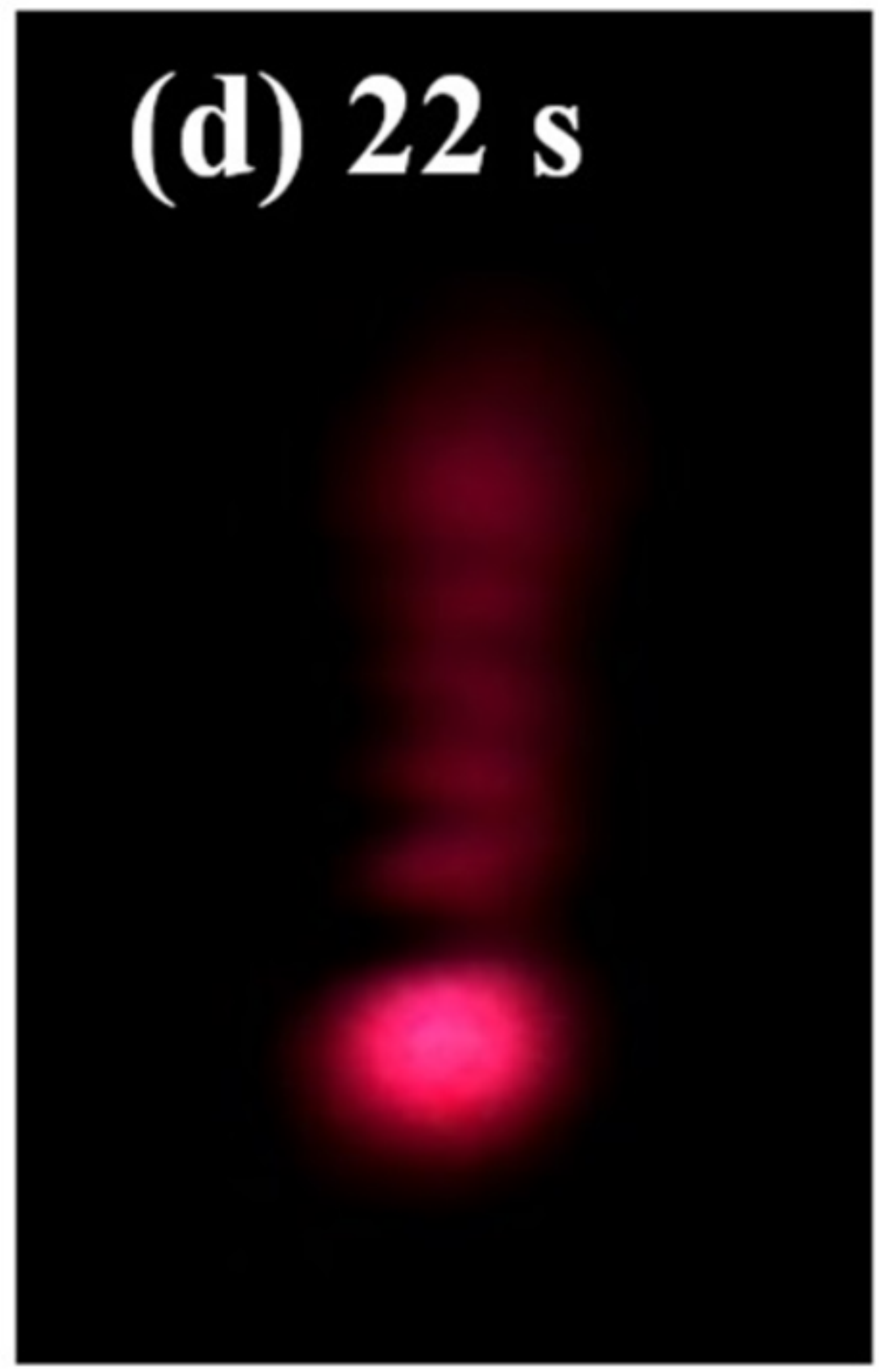




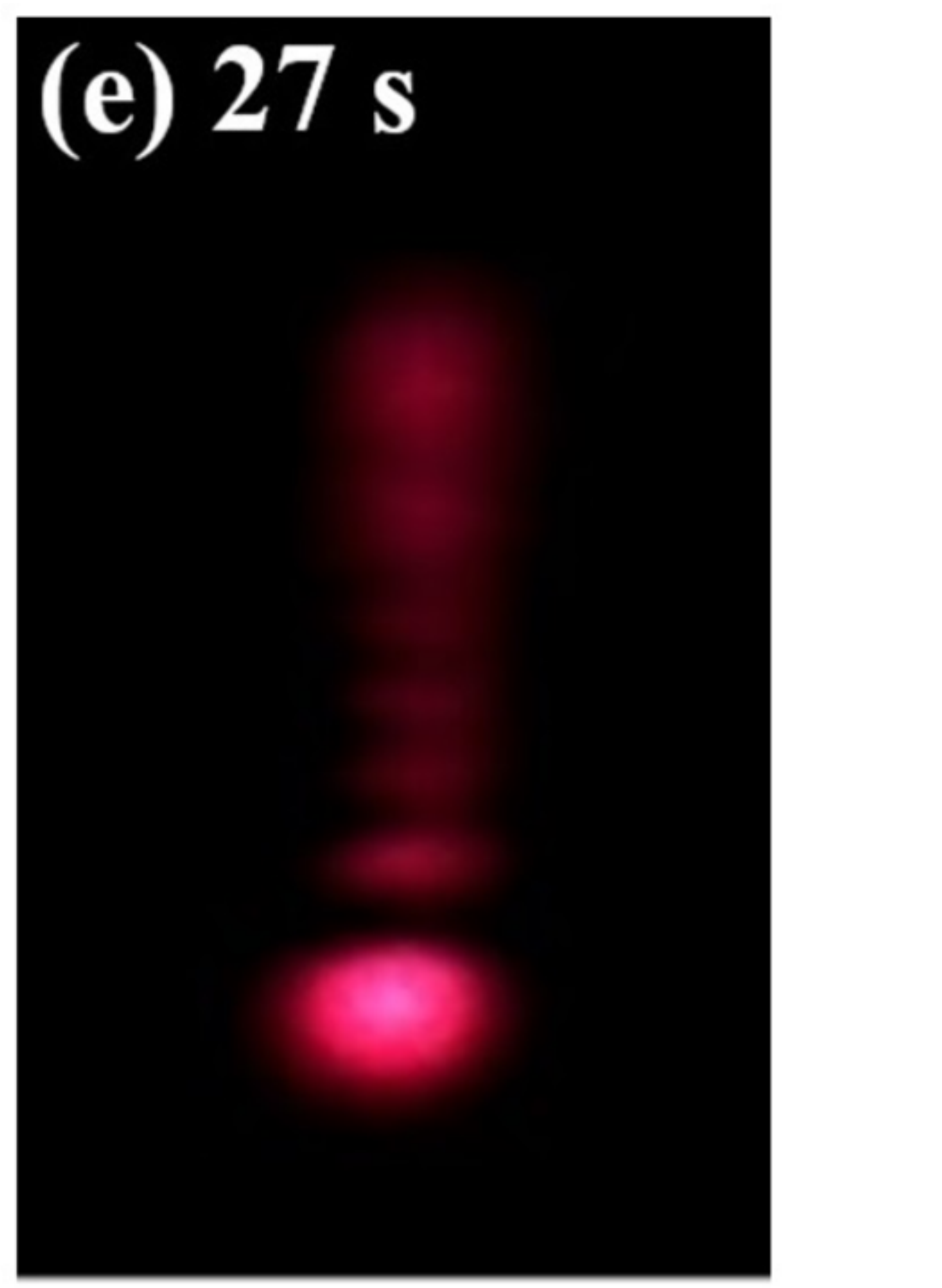

(e) $27 \mathrm{~s}$ e Click here to access/download;Figure;fig5e.jpg $\underline{\underline{\Perp}}$ 


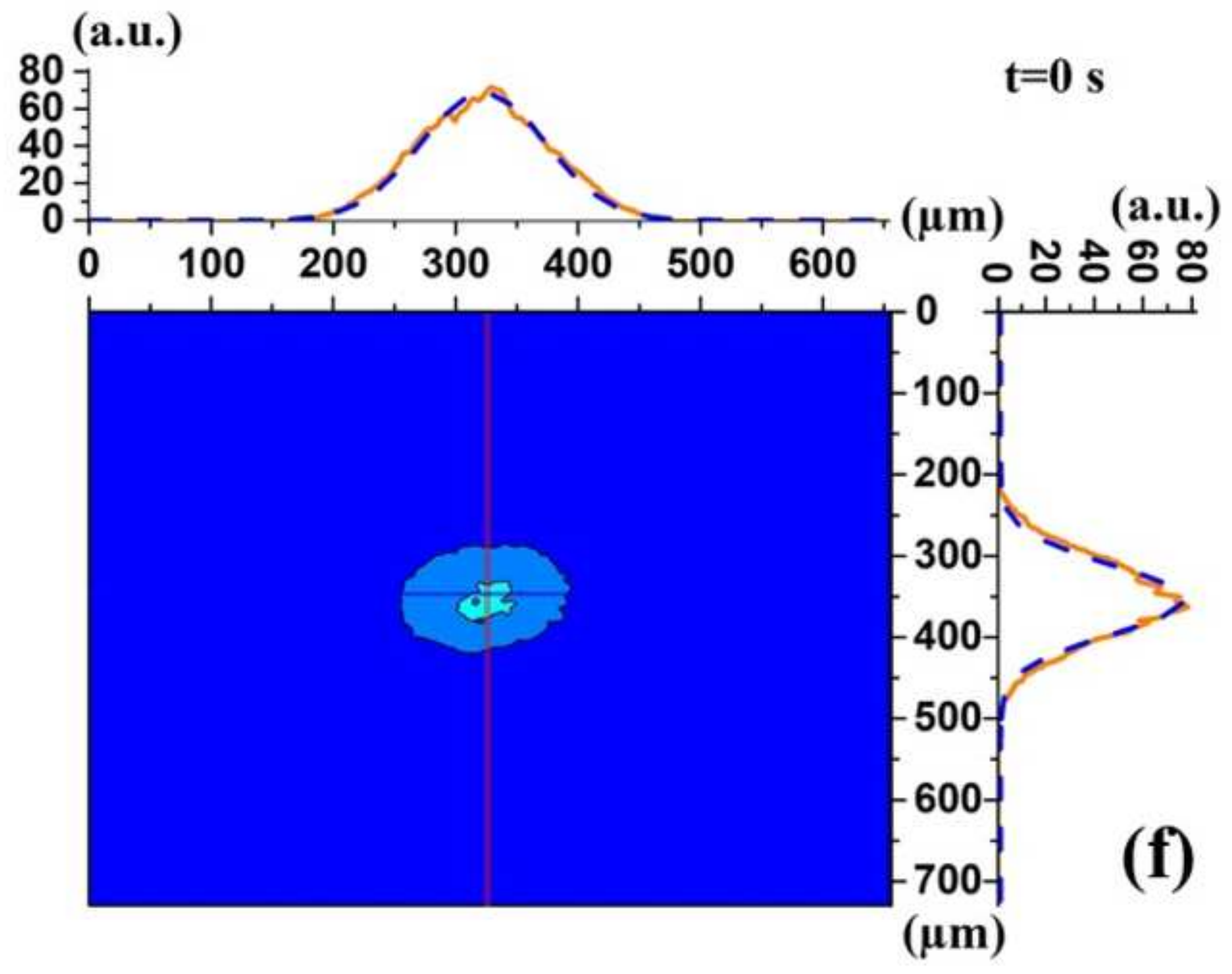



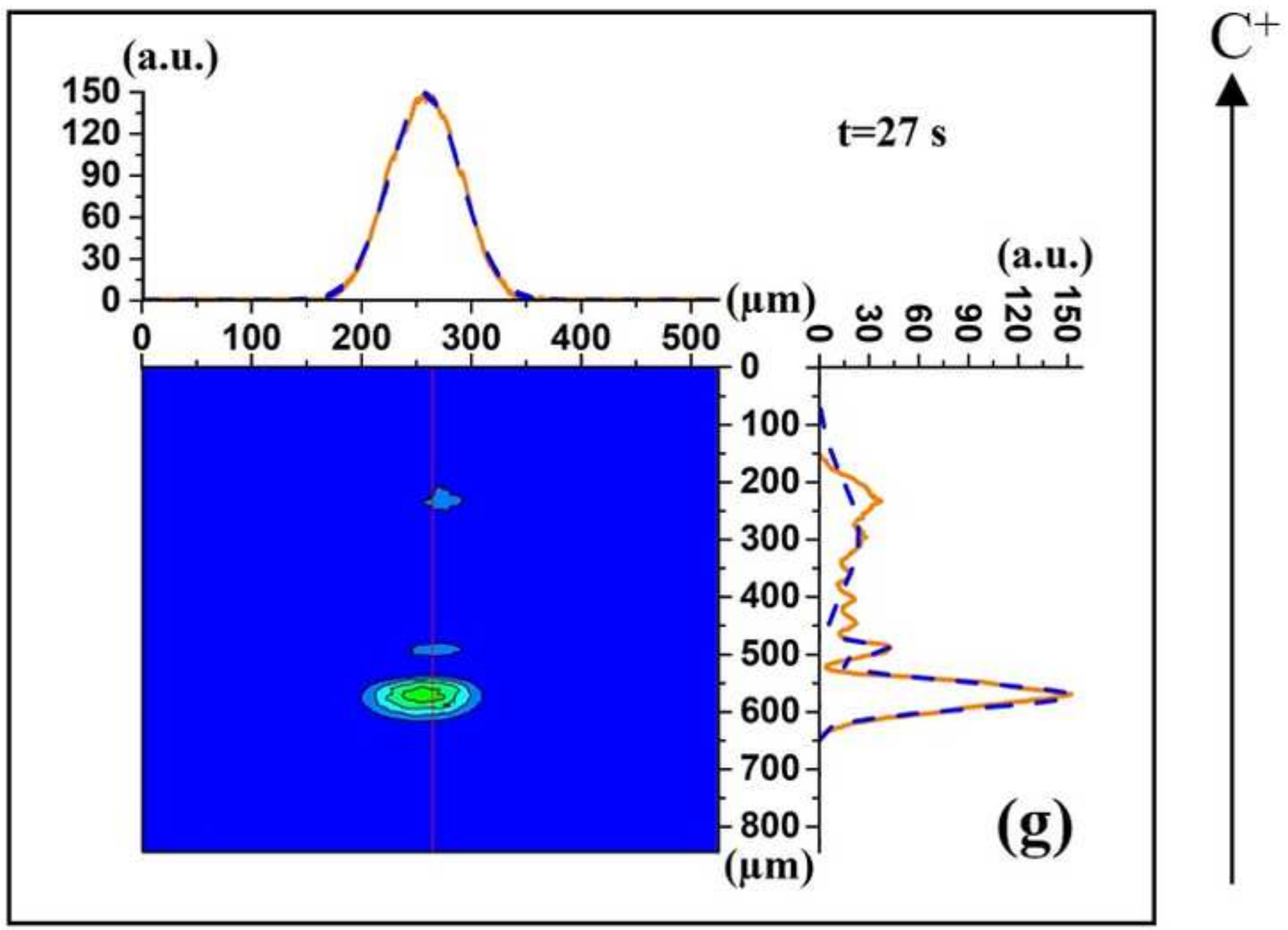


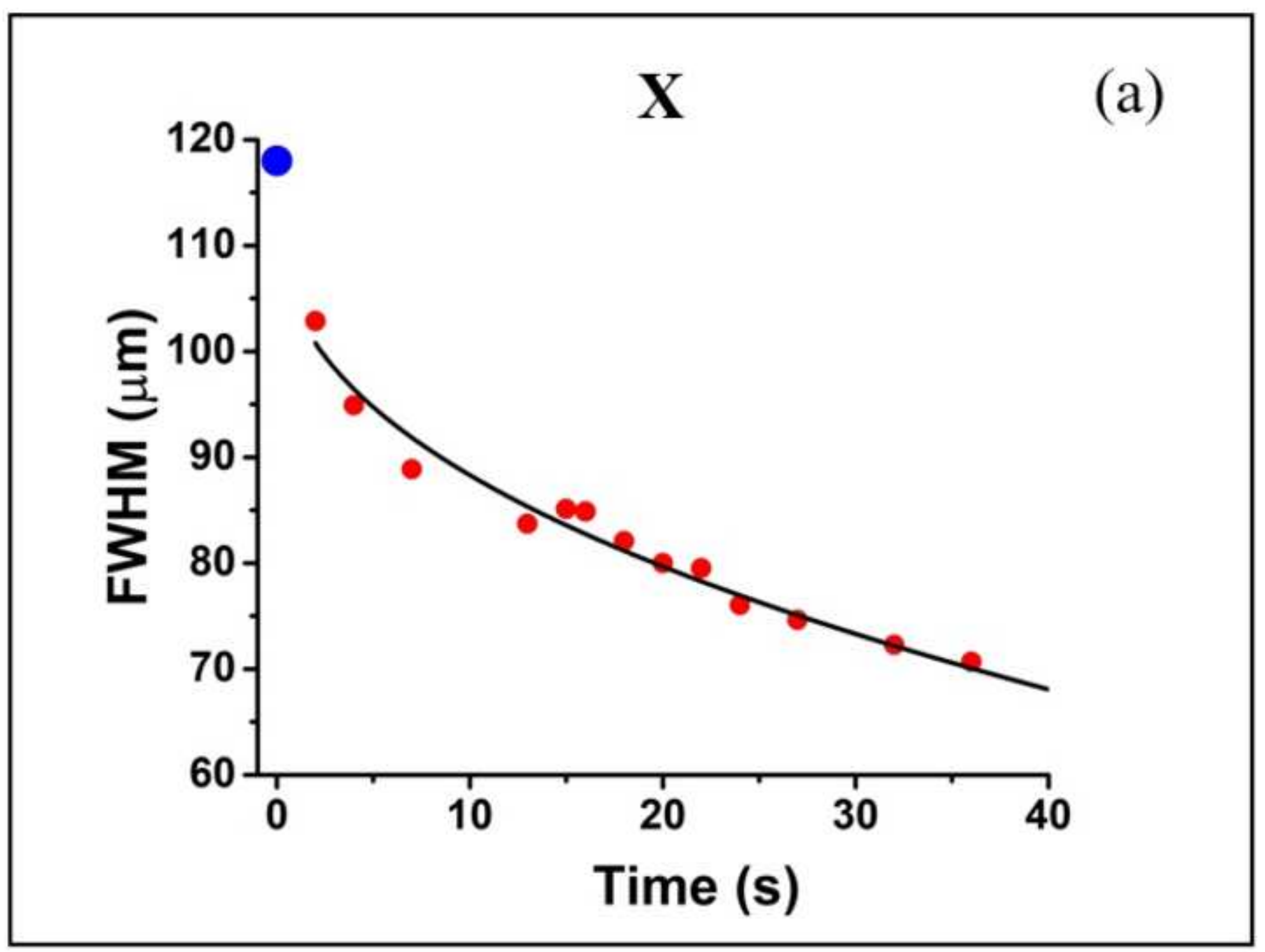




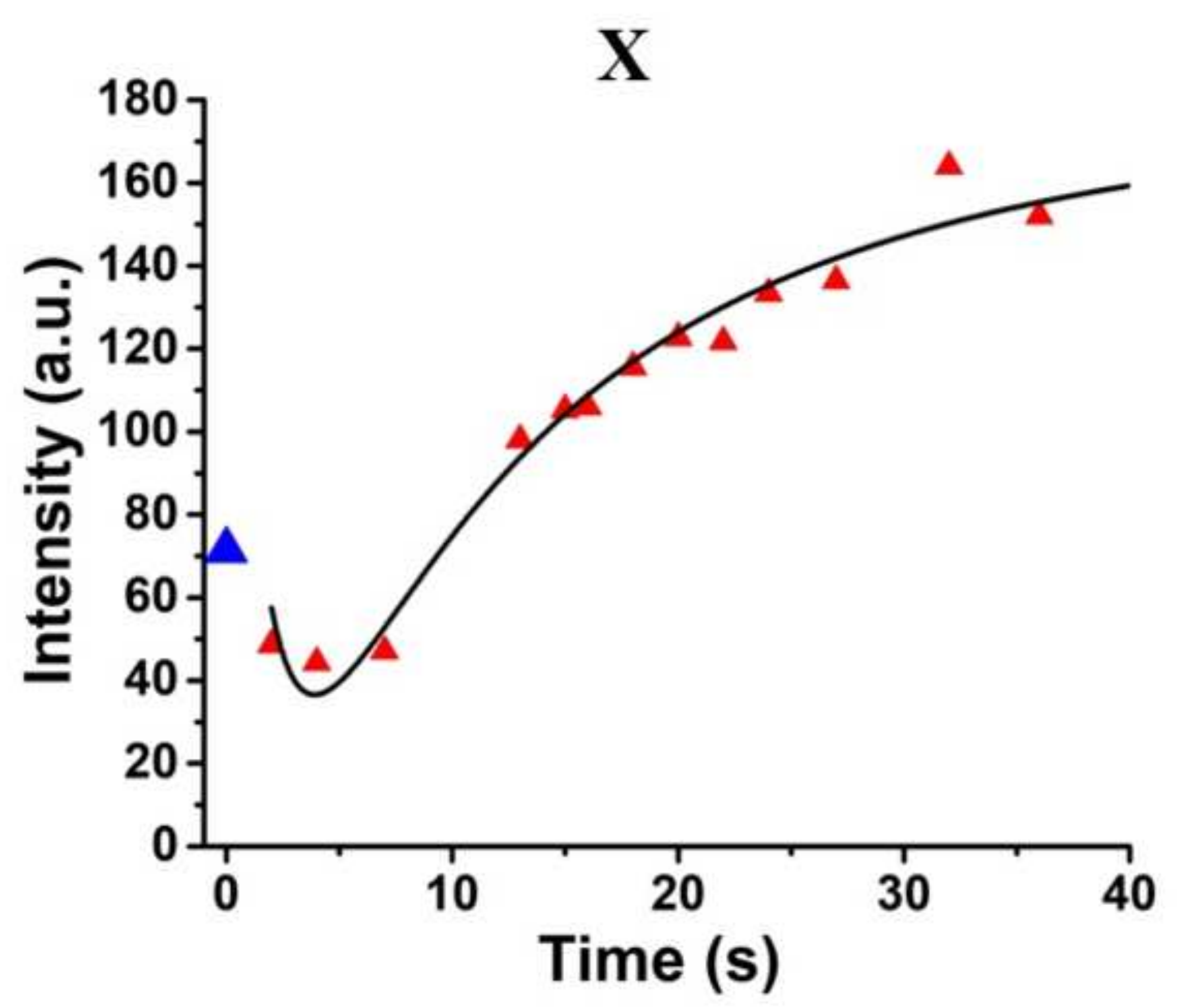

(b) 


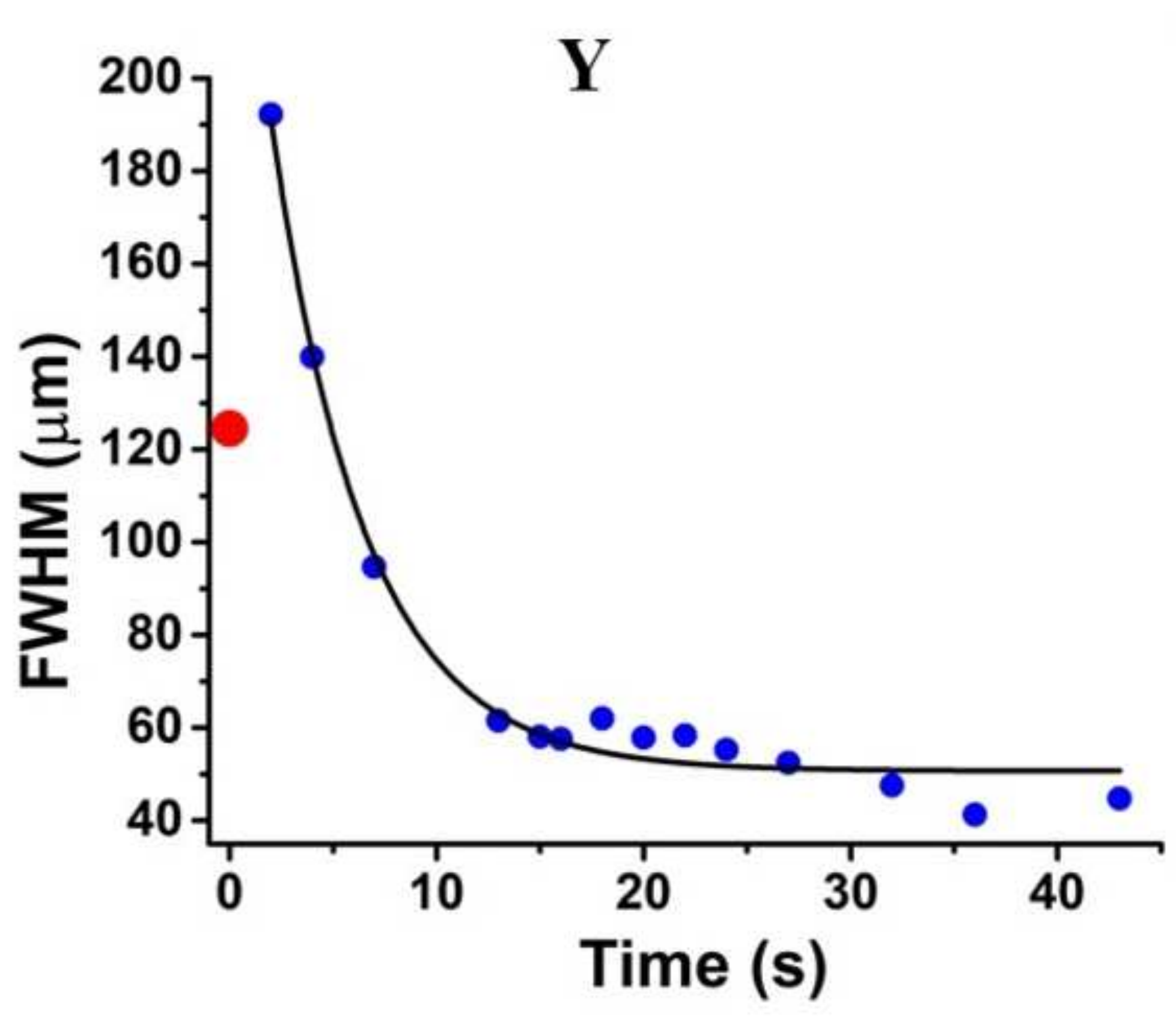

(c) 
Click here to access/download;Figure;fig6d.jpg $\underline{\underline{ \pm}}$

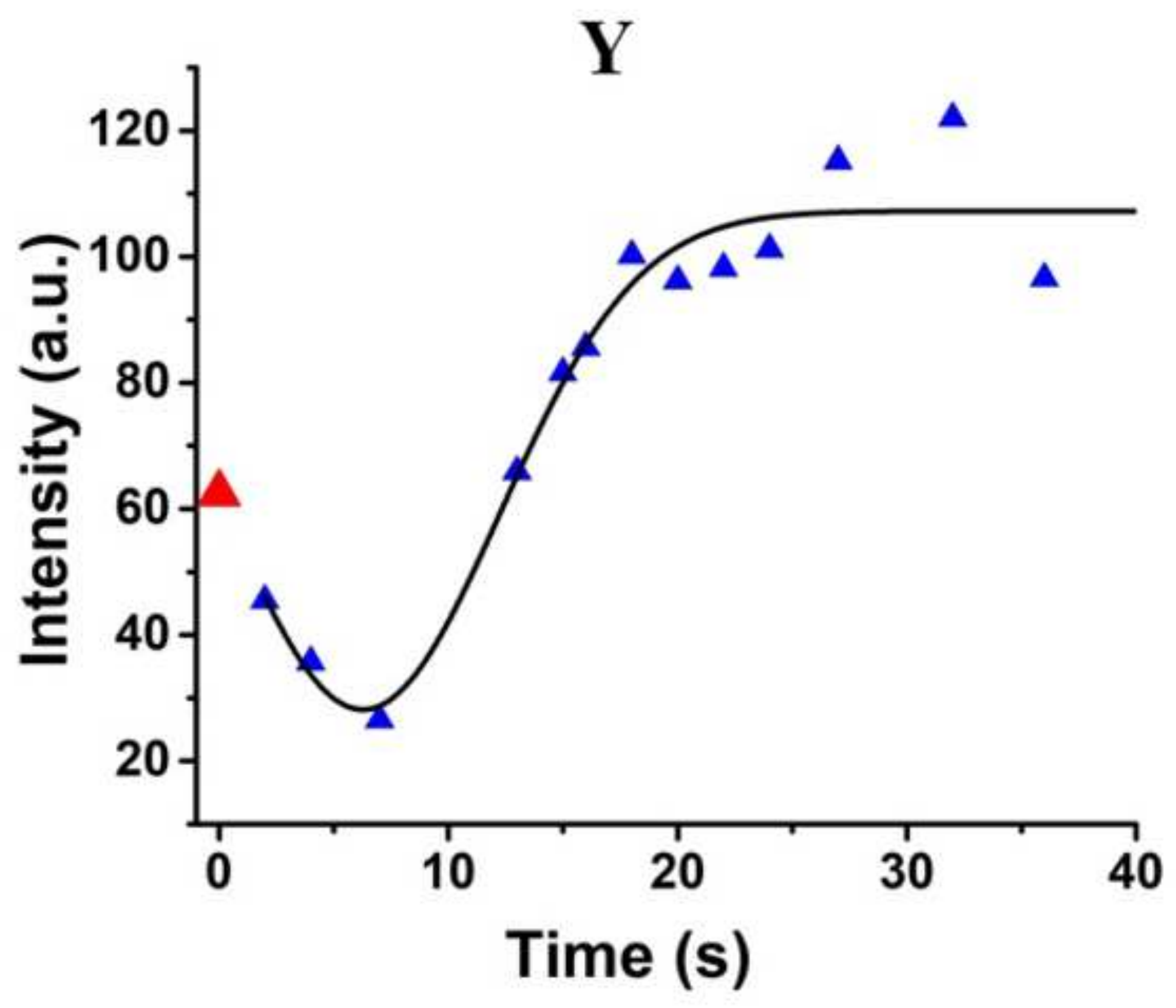

(d) 
Click here to access/download;Figure;fig7.jpg $\underline{\underline{ }}$

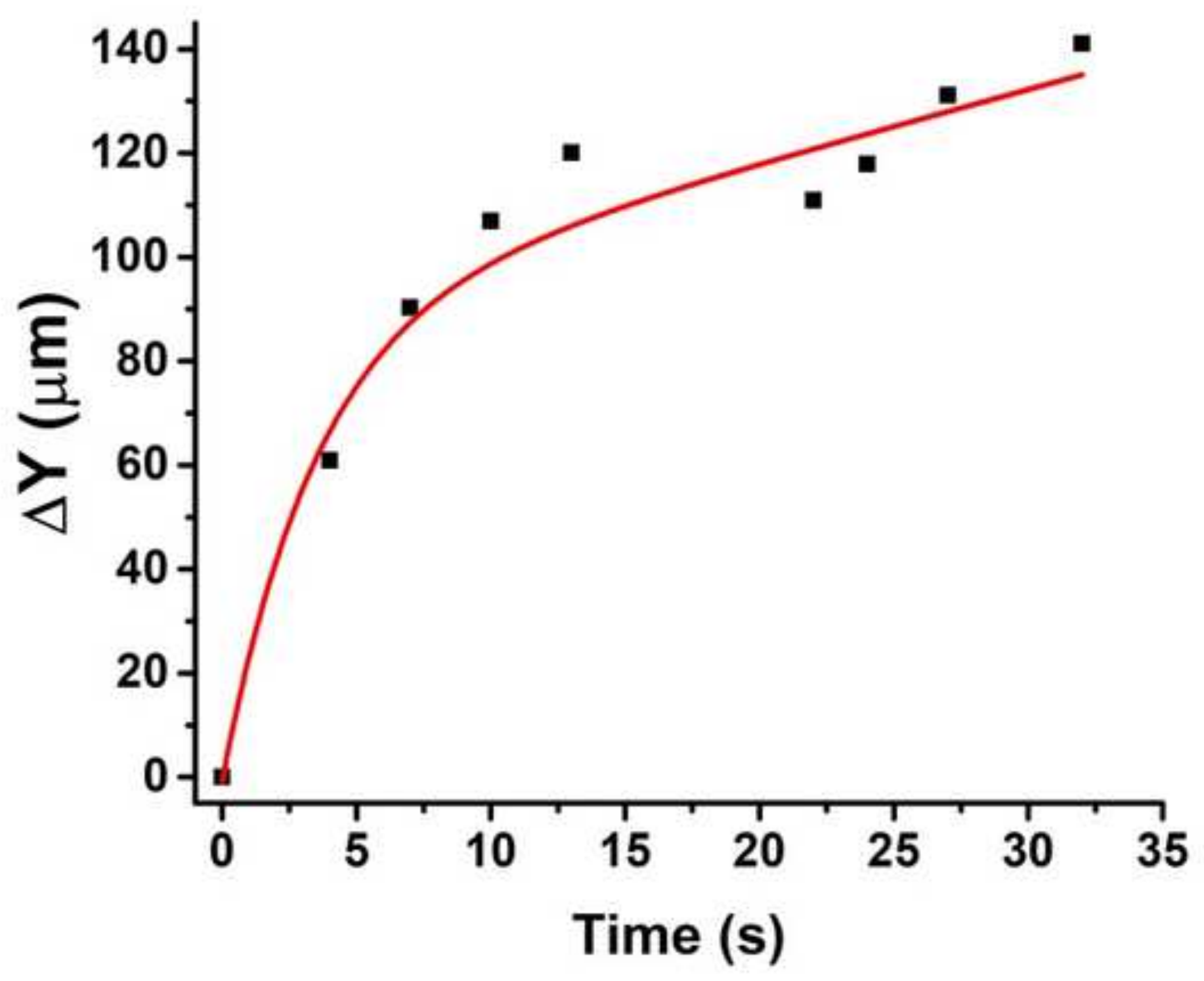




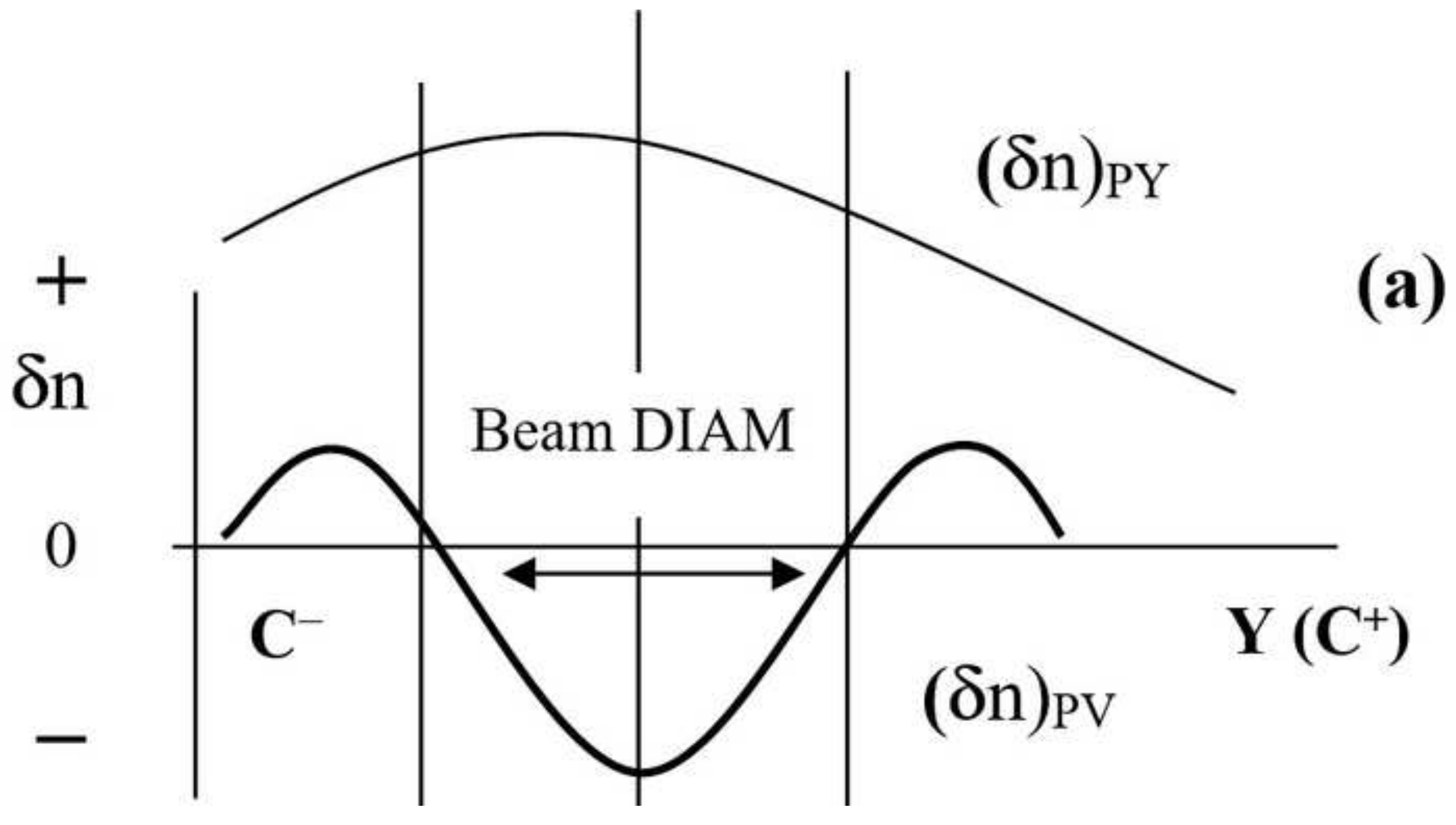




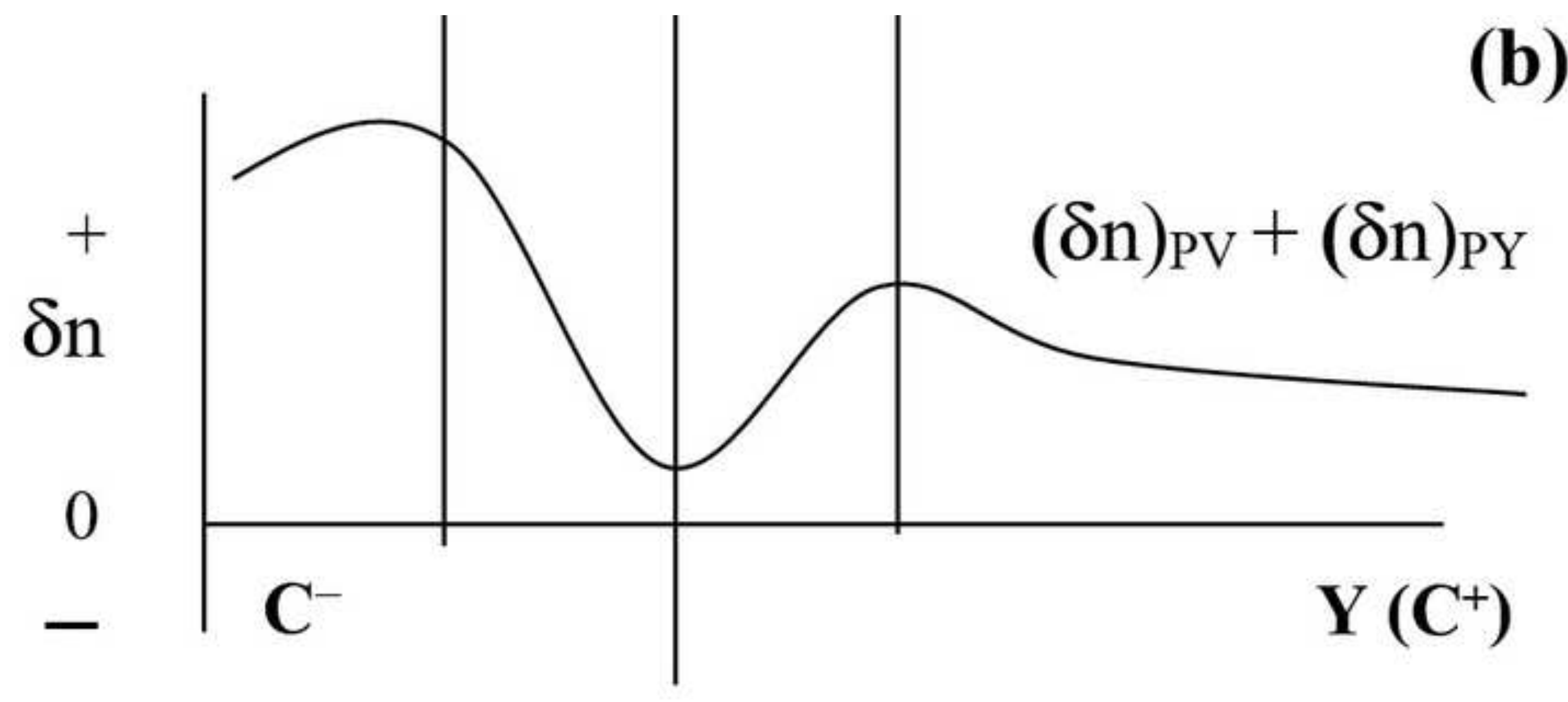




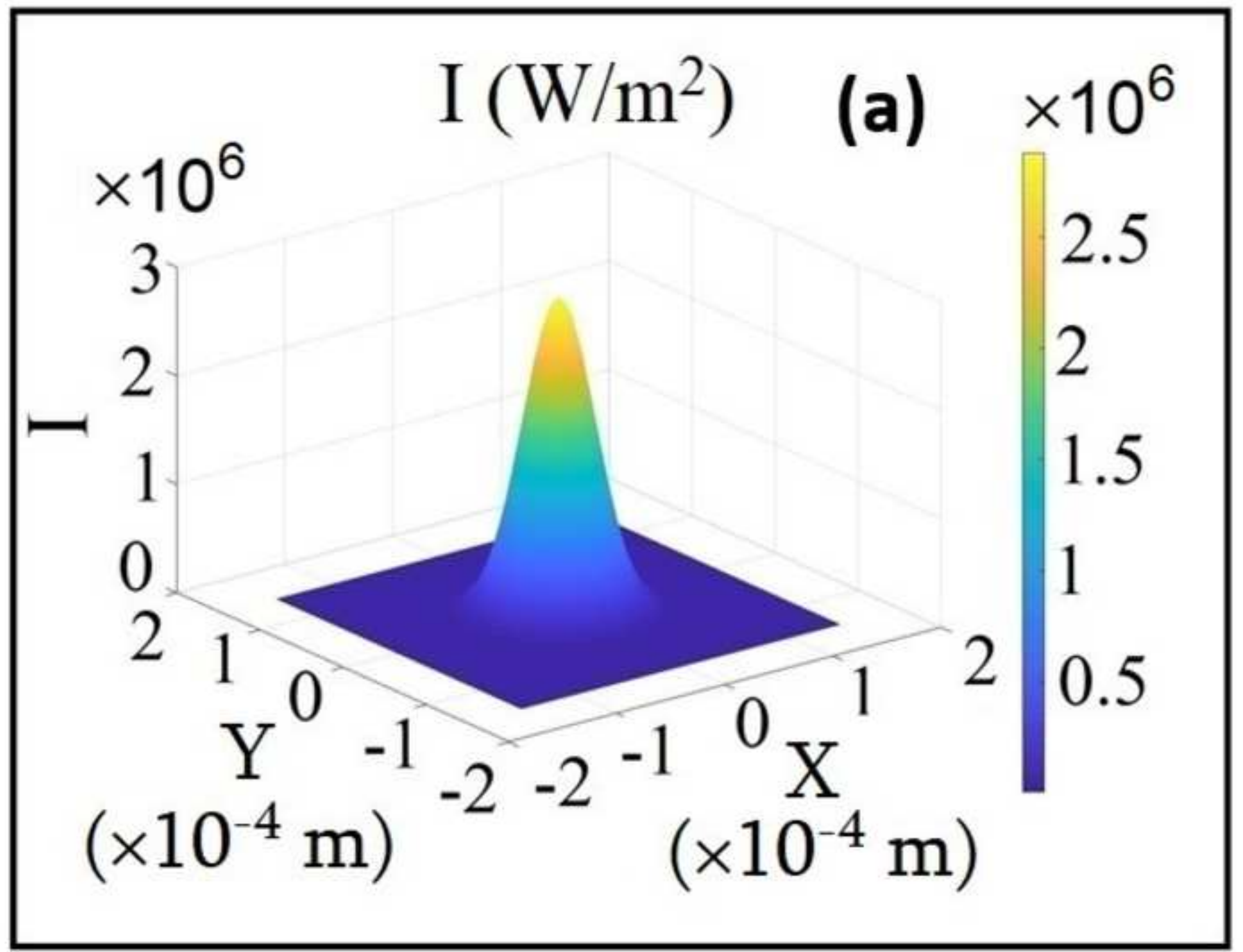




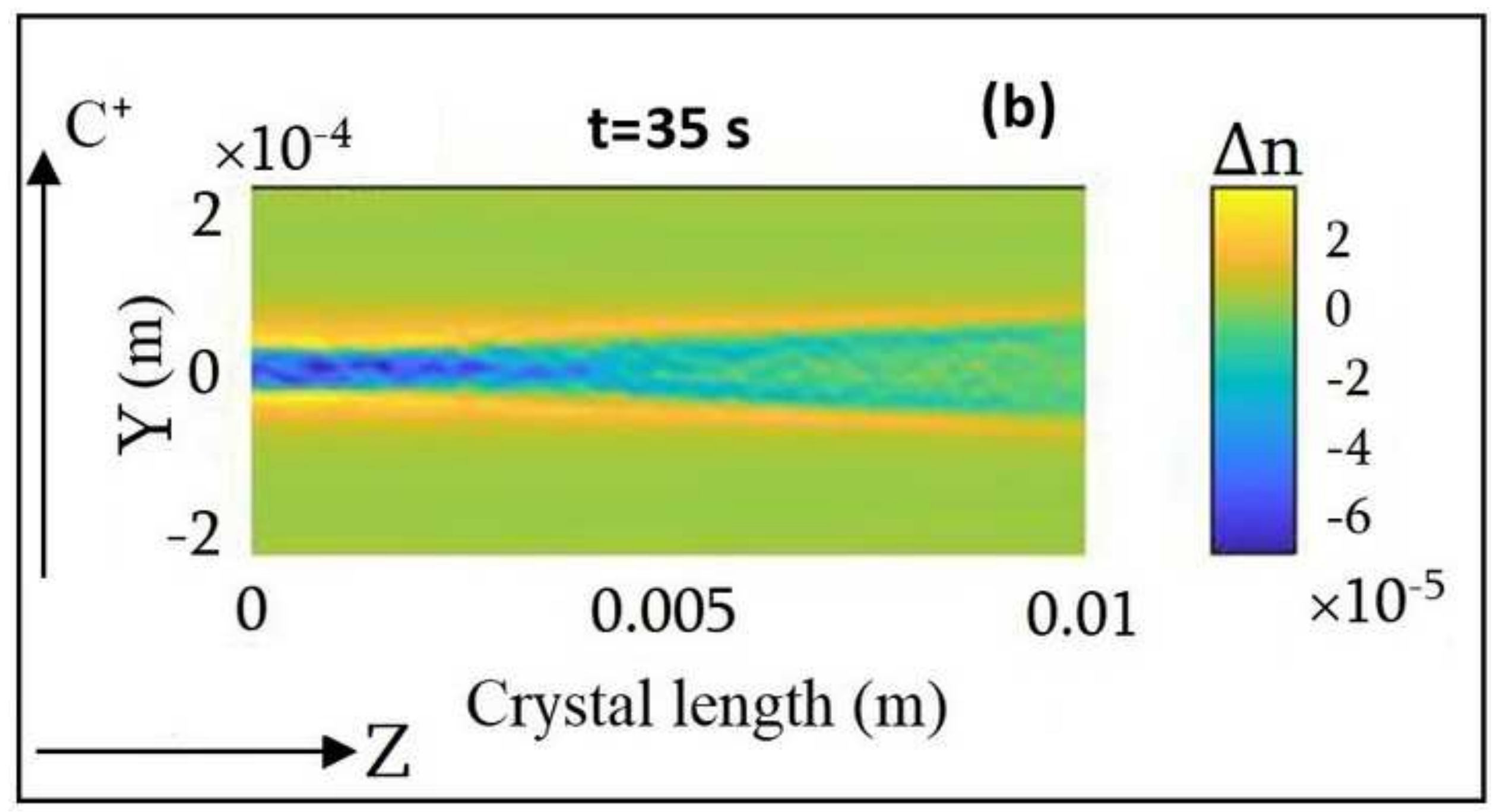




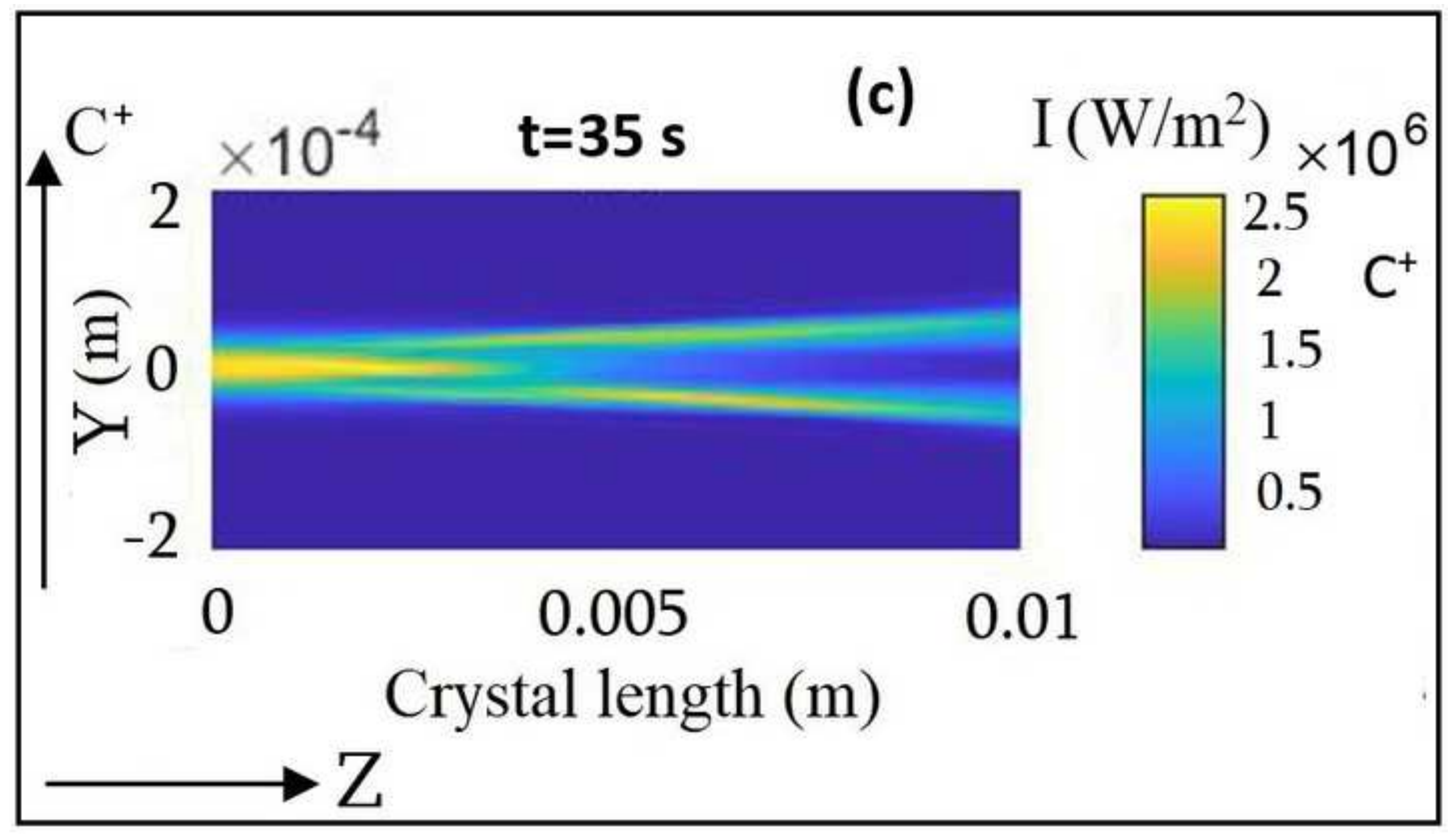




\section{(d)}

$\overbrace{-2}^{C^{+}}$

$t=35 s$

$\mathrm{I}\left(\mathrm{W} / \mathrm{m}^{2}\right)$ $\times 10^{5}$

$\times 10^{-4}$

2

2

$-2$

$-1$

0

1

$2 \times 10^{-4}$

$\mathrm{X}(\mathrm{m})$

12

10

8

6

4

2

$\longrightarrow \mathrm{X}$ 


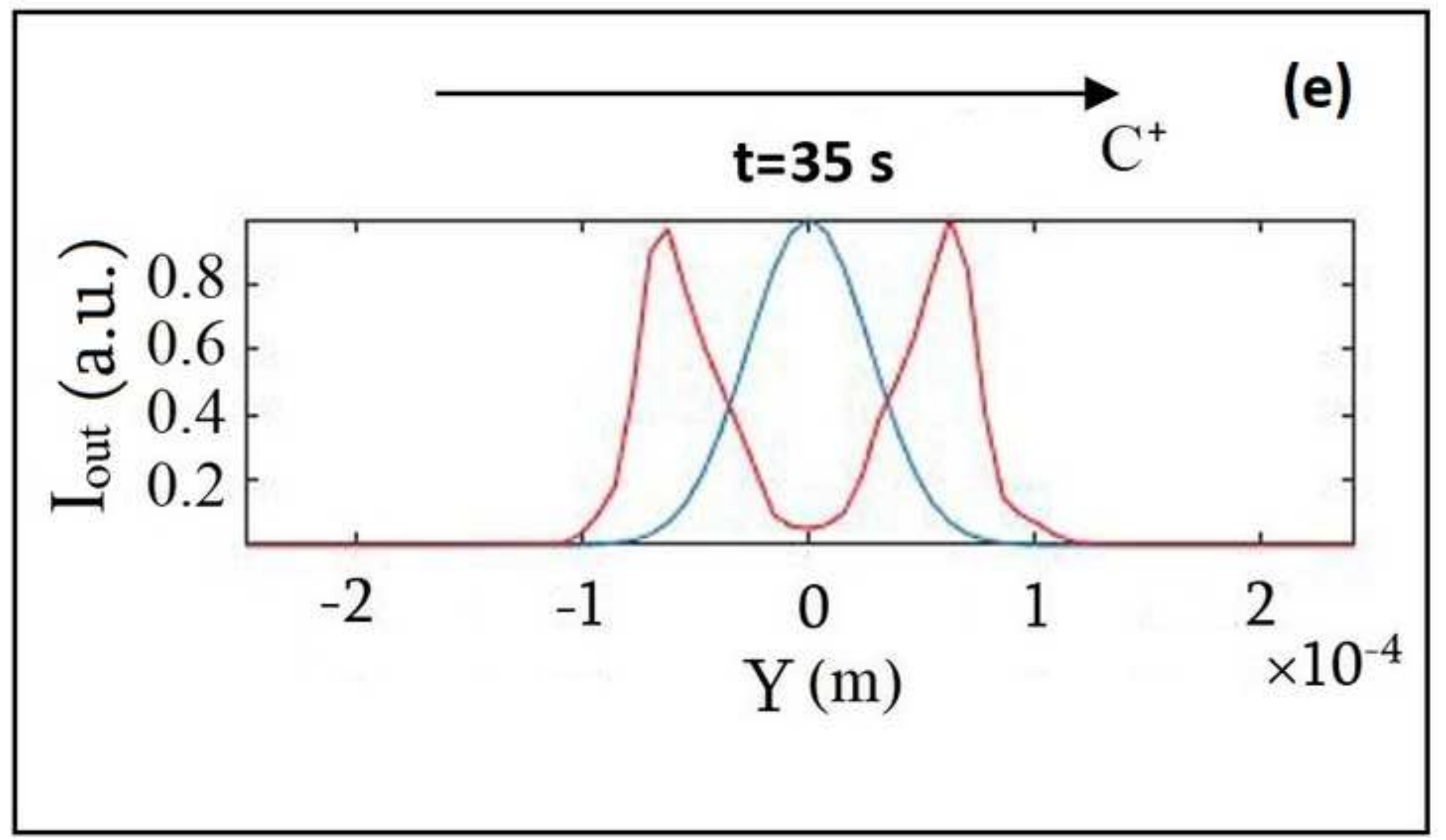




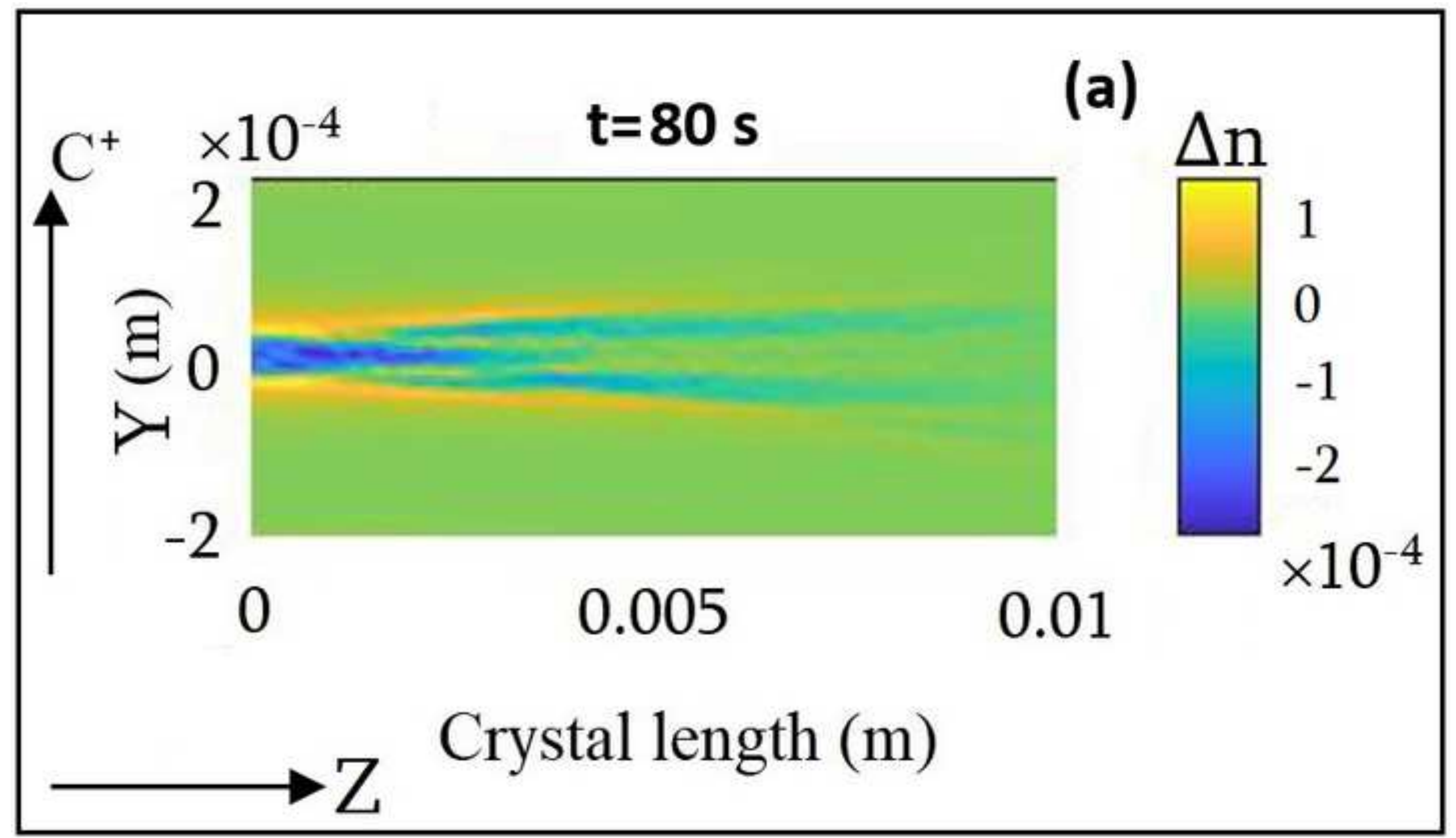




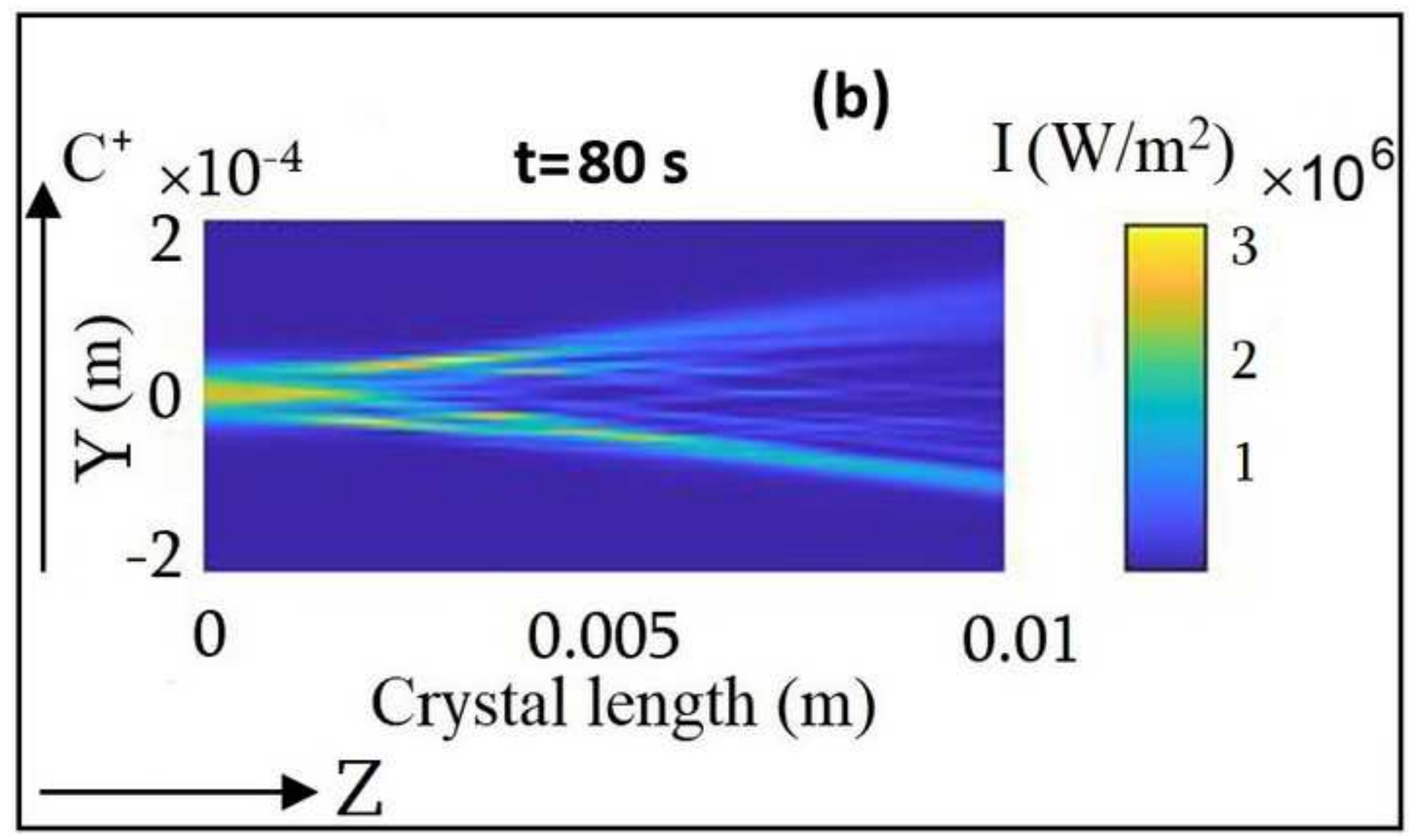




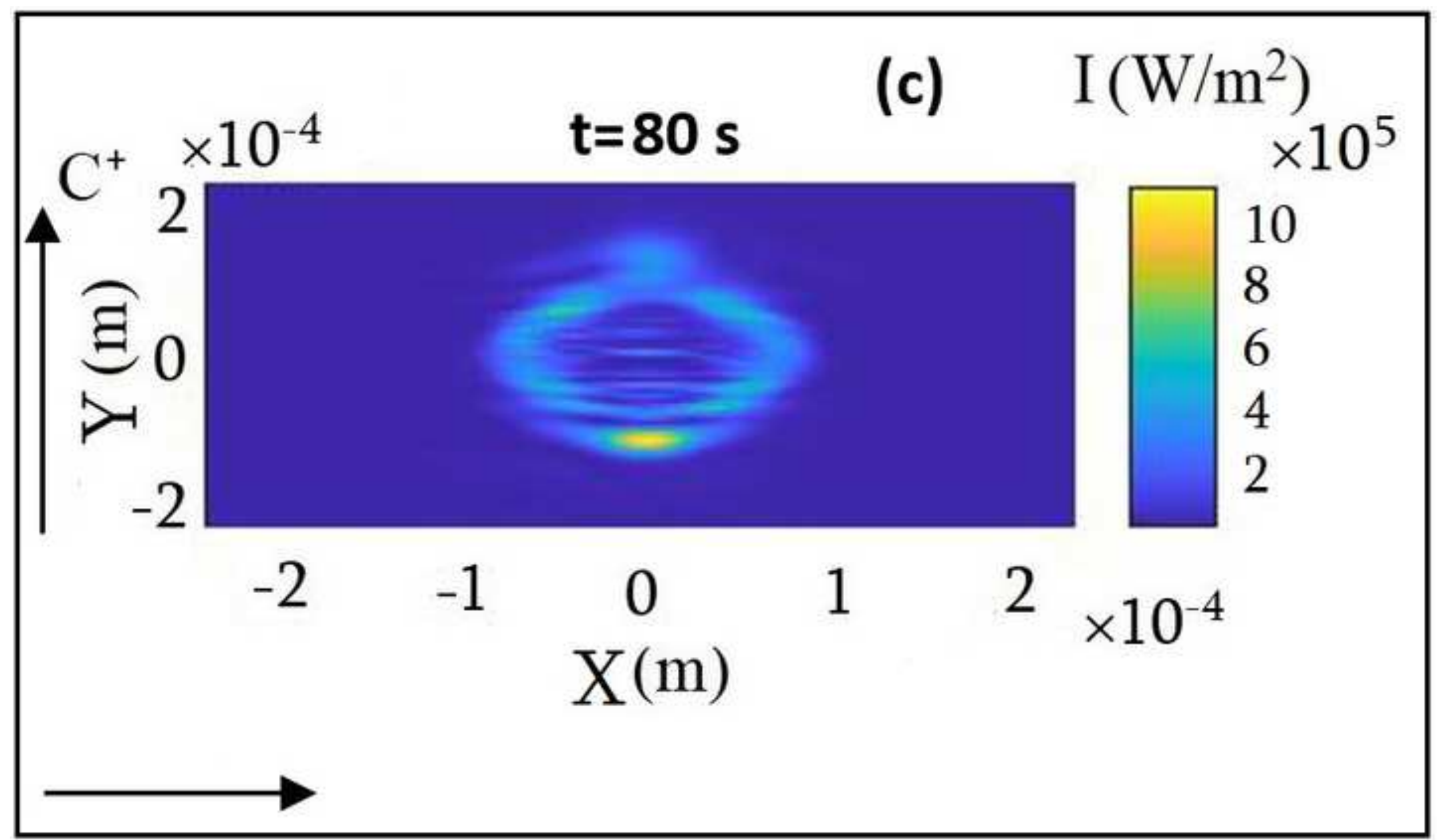




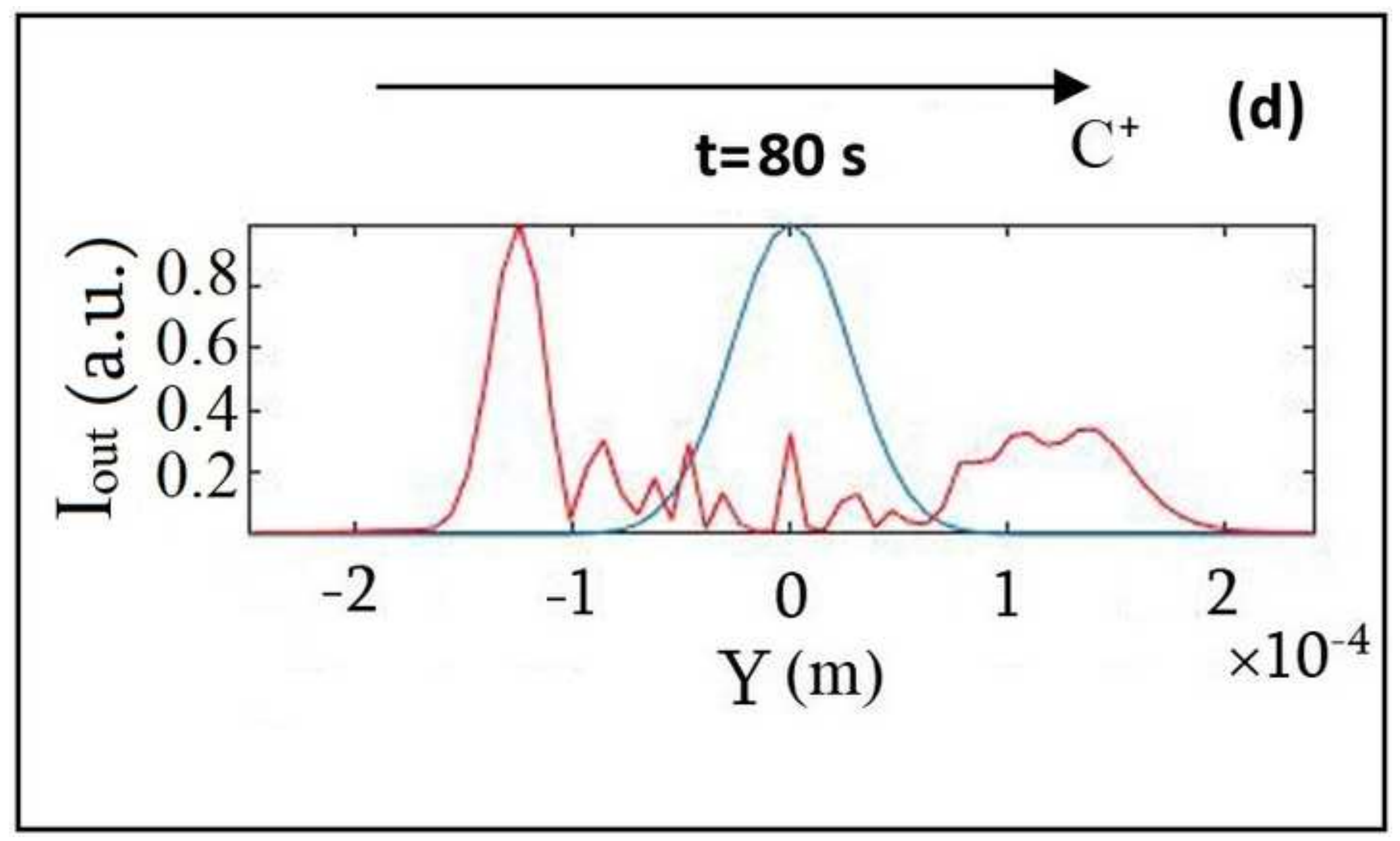


Click here to access/download;Figure;fig11.jpg $\underline{\underline{ }}$

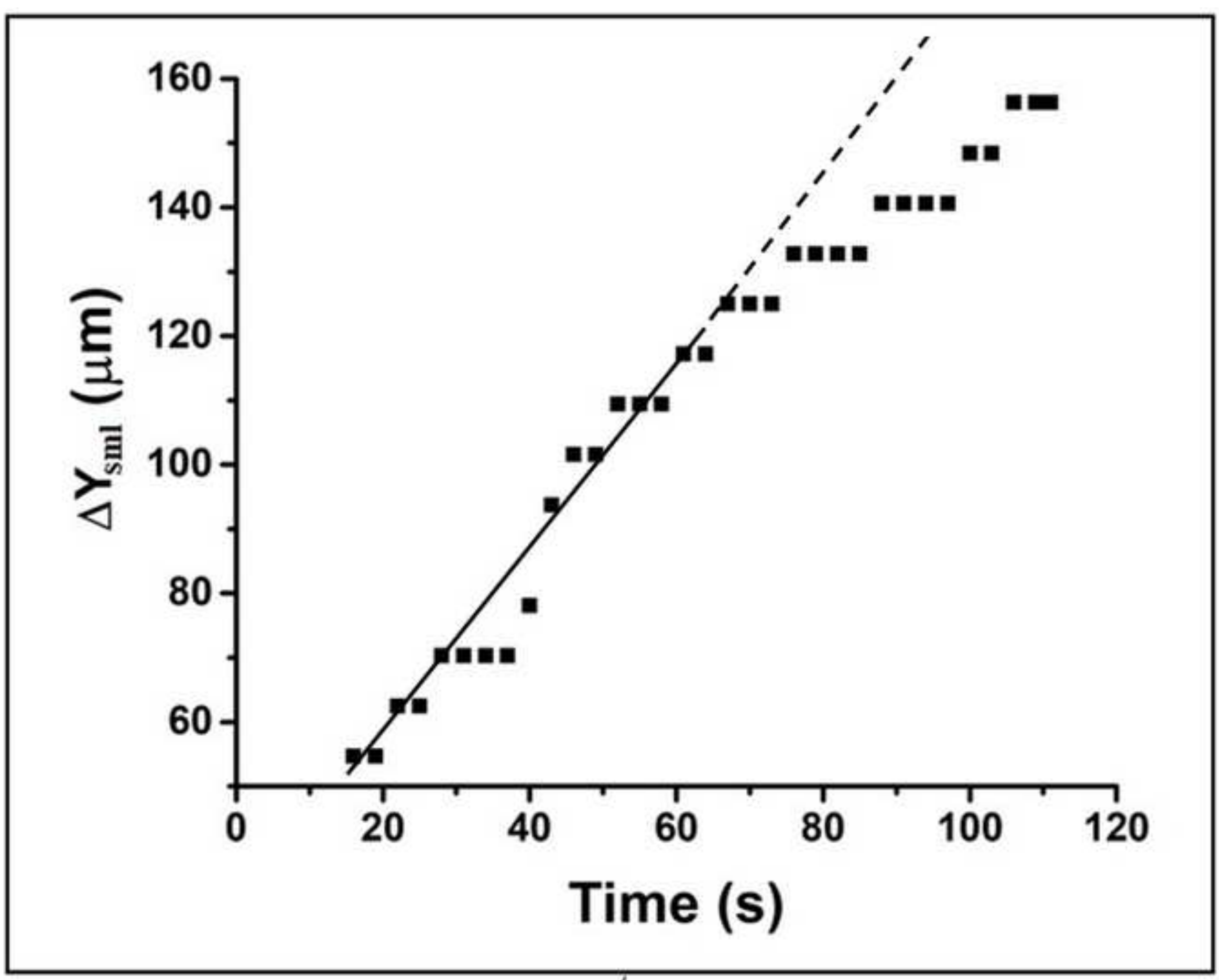




\title{
Pyroelectric-controlled bending of a self-trapped optical beam in a photorefractive iron doped lithium niobate crystal
}

\author{
Lusine Tsarukyan ${ }^{* \dagger}$, Anahit Badalyan ${ }^{\dagger}$, Fabrice Devaux ${ }^{\ddagger}$, \\ Mathieu Chauvet ${ }^{\star}$ and Rafael Drampyan ${ }^{\dagger}$ \\ ${ }^{\dagger}$ Institute for Physical Research, National Academy of Sciences of Armenia, 0203 Ashtarak-2, Armenia \\ *EMTO-ST Institute, University of Bourgogne Franche-Comte, F-25030, Besancon Cedex, France \\ *lustsarukyan@gmail.com
}

Table 1: Parameters for LN:Fe crystal involved in the numerical model.

\begin{tabular}{|c|c|c|}
\hline $\begin{array}{l}\text { Parameter } \\
(\lambda=633 \mathrm{~nm})\end{array}$ & Meaning & Value \\
\hline$\mu\left(\mathrm{m}^{2} \mathrm{~V}^{-1} \mathrm{~s}^{-1}\right)$ & Mobility of electric charges & $7.4 \times 10^{-5}$ \\
\hline$S_{F e}\left(\mathbf{m}^{2} \mathbf{J}^{-1}\right)$ & Cross-section of photoionization of donor & $3.3 \times 10^{-6}$ \\
\hline$S_{X}$ & Cross-section of photoionization of polarons & $5 \times 10^{-5}$ \\
\hline$S_{F e X}\left(\mathbf{m}^{5} \mathbf{J}^{-1}\right)$ & $\begin{array}{l}\text { Coupling efficiency between } \\
\text { polarons-donors }\end{array}$ & $3.22 \times 10^{-30}$ \\
\hline$\gamma_{F e}\left(\mathrm{~m}^{3} \mathrm{~s}^{-1}\right)$ & Recombination rate of electrons from $\mathrm{Fe}^{2+}$ & $1.65 \times 10^{-14}$ \\
\hline$\gamma_{X}$ & $\begin{array}{l}\text { Recombination rate of electrons } \\
\text { from } \mathrm{X}^{+} \text {-states }\end{array}$ & $5 \times 10^{-16}$ \\
\hline$\gamma_{X F e}$ & Recombination rate from $\mathrm{X}^{+}$states to $\mathrm{Fe}^{3+}$ & $1.14 \times 10^{-21}$ \\
\hline$K_{F e}^{o}\left(m^{3} V^{-1}\right)$ & $\begin{array}{l}\text { Photovoltaic coefficient of donors for } \\
o \text {-polarized light }\end{array}$ & $-3.5 \times 10^{-33}$ \\
\hline$K_{F e}{ }^{e}$ & $\begin{array}{l}\text { Photovoltaic coefficient of donors for } \\
e \text {-polarized light }\end{array}$ & $-7 \times 10^{-33}$ \\
\hline $\boldsymbol{K}_{X}{ }^{o}$ & $\begin{array}{l}\text { Photovoltaic coefficient of polarons for } \\
o \text {-polarized light }\end{array}$ & $-21.2 \times 10^{-33}$ \\
\hline$K_{X}^{e}$ & $\begin{array}{l}\text { Photovoltaic coefficient of polarons for } \\
e \text {-polarized light }\end{array}$ & $-42.4 \times 10^{-33}$ \\
\hline Fr._Fe & Fraction of ionized donors & 0.9 \\
\hline Fr. $X$ & Fraction of ionized polarons & 1.0 \\
\hline
\end{tabular}

\footnotetext{
* Corresponding author: lustsarukyan@gmail.com
} 